\title{
Variational integrators for constrained dynamical systems
}

\author{
Sigrid Leyendecker ${ }^{1,2, *}$, Jerrold E. Marsden ${ }^{1, * *}$, and Michael Ortiz ${ }^{2, * * *}$ \\ ${ }^{1}$ Control and Dynamical Systems, California Institute of Technology, USA \\ ${ }^{2}$ Graduate Aeronautical Laboratories, California Institute of Technology, USA
}

Received 28 December 2007, accepted 27 May 2008

Published online 10 September 2008

Key words Variational time integration, constrained dynamical systems, differential algebraic equations, flexible multibody dynamics.

\begin{abstract}
A variational formulation of constrained dynamics is presented in the continuous and in the discrete setting. The existing theory on variational integration of constrained problems is extended by aspects on the initialization of simulations, the discrete Legendre transform and certain postprocessing steps. Furthermore, the discrete null space method which has been introduced in the framework of energy-momentum conserving integration of constrained systems is adapted to the framework of variational integrators. It eliminates the constraint forces (including the Lagrange multipliers) from the timestepping scheme and subsequently reduces its dimension to the minimal possible number. While retaining the structure preserving properties of the specific integrator, the solution of the smaller dimensional system saves computational costs and does not suffer from conditioning problems. The performance of the variational discrete null space method is illustrated by numerical examples dealing with mass point systems, a closed kinematic chain of rigid bodies and flexible multibody dynamics and the solutions are compared to those obtained by an energy-momentum scheme.
\end{abstract}

() 2008 WILEY-VCH Verlag GmbH \& Co. KGaA, Weinheim

\section{Introduction}

The distinguishing feature of variational integrators is that time-stepping schemes are derived from a discrete variational principle based on a discrete action functional that approximates the continuous one. This is opposed to the standard derivation of integration methods that start with a continuous equation of motion (which itself might have been derived from a continuous variational principle) and replace the continuous quantities, in particular the derivatives with respect to time, by discrete approximations. The variational theory of discrete mechanics provides a theoretical framework that parallels continuous variational dynamics. Discrete analogues to the Euler-Lagrange equations, Noether's theorem, and the Legendre transform are derived from a discrete Lagrangian by performing similar steps as in the continuous theory. The resulting time-stepping schemes are structure preserving, i.e. they are symplectic-momentum conserving and exhibit good energy behavior, meaning that no artificial dissipation is present and the energy error stays bounded over long-time simulations. There exist many works on symplectic integrators like [1-7] to mention just a view. A detailed introduction plus a survey on the history and literature on the variational view of discrete mechanics is given in [8].

That work introduces also the theoretical background to deal with holonomically constrained systems in the framework of discrete variational mechanics by enforcing constraints using Lagrange multipliers or formulating the problem in generalized coordinates directly in the constraint manifold. A more applied approach to the variational integration of constrained problems, that already involves the idea of formulating the discrete Lagrangian in terms of a reparametrized redundant configuration variable, is given in [9]. However, many issues that are important from a practical point of view when using variational integrators for the simulation of constrained dynamics, like a consistent initialization or postprocessing steps necessary to evaluate the obtained discrete trajectory, have not been addressed yet.

The first purpose of this work is to fill this gap by providing details on the discrete Lagrangian, the discrete Legendre transform, the calculation of energy along a discrete trajectory for constrained problems and the fulfillment of constraints on configuration and on momentum level. Secondly the recently in the context of energy-momentum conserving time-stepping schemes developed discrete null space method [10] is adapted to the framework of variational integrators, in particular

* Corresponding author, e-mail: sleye@ caltech.edu, Phone: +1 6263958113, Fax: +1 6264496359

** Research partially supported by AFOSR grant FA9550-08-1-0173; e-mail: jmarsden@ caltech.edu

*** e-mail: ortiz@aero.caltech.edu 
it is shown that the resulting scheme is not only equivalent to the corresponding scheme using Lagrange multipliers, but that it can be derived itself via a discrete variational principle. Using the discrete null space method, the constraint forces are eliminated from the system and its dimension is reduced to the minimal possible number by the introduction of a discrete reparametrization in one time interval only. Besides leading to lower computational costs for many applications, this procedure removes the well-known conditioning problems associated with the use of Lagrange multipliers [11,12] while fulfilling constraints exactly. Thus it combines a number of advantageous properties compared to many other possibilities to handle constraints [13].

The discrete null space method has been applied successfully to the energy-momentum conserving integration of the dynamics of multibody systems consisting of rigid bodies connected by joints $[14,15]$ and to flexible multibody systems being composed of geometrically exact beams and shells $[15,16]$. Symplectic-momentum conserving integration of examples from both fields are presented in this work and the performance is compared to that using an energy-momentum scheme. For constrained problems, the energy-momentum conserving time-stepping equations involve the evaluation of more terms at the unknown configuration, wherefore the linearization is more complicated and the iterative solution is computationally more expensive.

No attempt to place the employed constrained formulation of rigid body and beam dynamics in the existing literature is made in this introduction since such surveys can be found in the introductions of the works cited in the corresponding sections.

This paper starts with a presentation of continuous constrained Lagrangian dynamics in Sect. 2. Then, the discrete counterparts are shown in Sect.3. In particular, the discrete null space method with nodal reparametrization is introduced in the variational framework and the questions of consistent initialization, constrained discrete Legendre transforms and hidden constraints are addressed. The developed theoretical aspects are illustrated by the simple example of a mathematical pendulum in Sect. 3.1. Sect. 4 recalls the formulation of rigid body dynamics in terms of a constrained configuration variable and presents the null space matrix and nodal reparametrization required for the integration using the discrete null space method. The procedure is extended to rigid multibody systems in Sect. 5 and an example of a closed kinematic chain is investigated in detail. Finally, aspects of geometrically exact beam dynamics in the framework of variational integration are discussed in Sect. 6, an example of a multibody system, consisting of a beam and rigid bodies is investigated and the results are compared to the those obtained using an energy-momentum scheme.

\section{Constrained Lagrangian dynamics}

Consider an $n$-dimensional mechanical system in a configuration manifold $Q \subseteq \mathbb{R}^{n}$ with configuration vector $\boldsymbol{q}(t) \in Q$ and velocity vector $\dot{\boldsymbol{q}}(t) \in T_{\boldsymbol{q}(t)} Q$, where $t$ denotes the time variable in the bounded interval $\left[t_{0}, t_{N}\right] \subset \mathbb{R}$. In general, the Lagrangian of a mechanical system consists of the difference of the kinetic energy $T(\dot{\boldsymbol{q}})$ and a potential $V(\boldsymbol{q})$ accounting for elastic deformation and for external loading (if present). Let the motion be constrained by the vector valued function of holonomic, scleronomic constraints requiring $\boldsymbol{g}(\boldsymbol{q})=\mathbf{0} \in \mathbb{R}^{m}$. It is assumed that $\mathbf{0} \in \mathbb{R}^{m}$ is a regular value of the constraints, such that

$$
C=\boldsymbol{g}^{-1}(\mathbf{0})=\{\boldsymbol{q} \mid \boldsymbol{q} \in Q, \boldsymbol{g}(\boldsymbol{q})=\mathbf{0}\} \subset Q
$$

is an $(n-m)$-dimensional submanifold, called constraint manifold. Just as $C$ can be embedded in $Q$ via $i: C \rightarrow Q$, its $2(n-m)$-dimensional tangent bundle

$$
T C=\left\{(\boldsymbol{q}, \dot{\boldsymbol{q}}) \mid(\boldsymbol{q}, \dot{\boldsymbol{q}}) \in T_{\boldsymbol{q}} Q, \boldsymbol{g}(\boldsymbol{q})=\mathbf{0}, \boldsymbol{G}(\boldsymbol{q}) \cdot \dot{\boldsymbol{q}}=\mathbf{0}\right\} \subset T Q
$$

can be embedded in $T Q$ in a natural way by tangent lift $T i: T C \rightarrow T Q$. Here and in the sequel $\boldsymbol{G}(\boldsymbol{q})=D \boldsymbol{g}(\boldsymbol{q})$ denotes the $m \times n$ Jacobian of the constraints. Note that admissible velocities are constrained to the null space of the constraint Jacobian.

A Lagrangian $L: T Q \rightarrow \mathbb{R}$ can be restricted to $L^{C}=L_{\mid T C}: T C \rightarrow \mathbb{R}$. To investigate the relation of the dynamics of $L^{C}$ on $T C$ and the dynamics of $L$ on $T Q$, the following notation is used. $\mathcal{C}(Q)=\mathcal{C}\left(\left[t_{0}, t_{N}\right], Q, \boldsymbol{q}_{0}, \boldsymbol{q}_{N}\right)$ denotes the space of smooth functions satisfying $\boldsymbol{q}\left(t_{0}\right)=\boldsymbol{q}_{0}$ and $\boldsymbol{q}\left(t_{N}\right)=\boldsymbol{q}_{N}$, where $\boldsymbol{q}_{0}, \boldsymbol{q}_{N} \in C \subset Q$ are fixed endpoints. Let $\mathcal{C}(C)$ denote the corresponding space of curves in $C$ and set $\mathcal{C}\left(\mathbb{R}^{m}\right)=\mathcal{C}\left(\left[t_{0}, t_{N}\right], \mathbb{R}^{m}\right)$ to be the space of curves $\lambda:\left[t_{0}, t_{N}\right] \rightarrow \mathbb{R}^{m}$ with no boundary conditions. This notation has been introduced in [8], where a large part of the theory presented here can be found.

Theorem 2.1 Suppose that 0 is a regular value of the scleronomic holonomic constraints $\boldsymbol{g}: Q \rightarrow \mathbb{R}^{m}$ and set $C=\boldsymbol{g}^{-1}(\mathbf{0}) \subset Q$. Let $L: T Q \rightarrow \mathbb{R}$ be a Lagrangian and $L^{C}=L_{\mid T C}$ its restriction to $T C$. Then the following statements are equivalent:

(i) $\boldsymbol{q} \in \mathcal{C}(C)$ extremizes the action integral $S^{C}(\boldsymbol{q})=\int_{t_{0}}^{t_{N}} L^{C}(\boldsymbol{q}, \dot{\boldsymbol{q}}) d t$ and hence solves the Euler-Lagrange equations for $L^{C}$. 
(ii) $\boldsymbol{q} \in \mathcal{C}(Q)$ and $\boldsymbol{\lambda} \in \mathcal{C}\left(\mathbb{R}^{m}\right)$ satisfy the constrained Euler-Lagrange equations

$$
\begin{aligned}
\frac{\partial L(\boldsymbol{q}, \dot{\boldsymbol{q}})}{\partial \boldsymbol{q}}-\frac{d}{d t}\left(\frac{\partial L(\boldsymbol{q}, \dot{\boldsymbol{q}})}{\partial \dot{\boldsymbol{q}}}\right)-\boldsymbol{G}^{T}(\boldsymbol{q}) \cdot \boldsymbol{\lambda} & =\mathbf{0} \\
\boldsymbol{g}(\boldsymbol{q}) & =\mathbf{0}
\end{aligned}
$$

(iii) $(\boldsymbol{q}, \boldsymbol{\lambda}) \in \mathcal{C}\left(Q \times \mathbb{R}^{m}\right)$ extremize $\bar{S}(\boldsymbol{q}, \boldsymbol{\lambda})=S(\boldsymbol{q})-\langle\boldsymbol{\lambda}, \boldsymbol{g}(\boldsymbol{q})\rangle$ and hence, solve the Euler-Lagrange equations for the augmented Lagrangian $\bar{L}: T\left(Q \times \mathbb{R}^{m}\right) \rightarrow \mathbb{R}$ defined by

$$
\bar{L}(\boldsymbol{q}, \boldsymbol{\lambda}, \dot{\boldsymbol{q}}, \dot{\boldsymbol{\lambda}})=L(\boldsymbol{q}, \dot{\boldsymbol{q}})-\boldsymbol{g}^{T}(\boldsymbol{q}) \cdot \boldsymbol{\lambda} .
$$

The proof given in [8] makes use of the Lagrange multiplier theorem (see e.g. [17]). The term $-\boldsymbol{G}^{T}(\boldsymbol{q}) \cdot \boldsymbol{\lambda} \in(T C)^{\perp}$ in $(3)_{1}$ represents the constraint forces that prevent the system from deviation of the constraint manifold. See Corollary 3.5 for further explanation on the space $(T C)^{\perp}$.

The continuous null space method. For every $\boldsymbol{q} \in C$, the basis vectors of $T_{\boldsymbol{q}} C$ form an $n \times(n-m)$ matrix $\boldsymbol{P}(\boldsymbol{q})$ with corresponding linear map $\boldsymbol{P}(\boldsymbol{q}): \mathbb{R}^{n-m} \rightarrow T_{\boldsymbol{q}} C$. This matrix is called null space matrix, since

$$
\operatorname{range}(\boldsymbol{P}(\boldsymbol{q}))=\operatorname{null}(\boldsymbol{G}(\boldsymbol{q}))=T_{\boldsymbol{q}} C \text {. }
$$

Hence admissible velocities can be expressed as

$$
\dot{\boldsymbol{q}}(t)=\boldsymbol{P}(\boldsymbol{q}) \cdot \boldsymbol{\nu}(t)
$$

with the independent generalized (quasi-) velocities $\nu \in \mathbb{R}^{n-m}$. Thus a premultiplication of the differential Eq. (3) $)_{1}$ by $\boldsymbol{P}^{T}(\boldsymbol{q})$ eliminates the constraint forces including the Lagrange multipliers from the system. The resulting d'Alembert-type equations of motion read

$$
\begin{aligned}
\boldsymbol{P}^{T}(\boldsymbol{q}) \cdot\left[\frac{\partial L(\boldsymbol{q}, \dot{\boldsymbol{q}})}{\partial \boldsymbol{q}}-\frac{d}{d t}\left(\frac{\partial L(\boldsymbol{q}, \dot{\boldsymbol{q}})}{\partial \dot{\boldsymbol{q}}}\right)\right] & =\mathbf{0} \\
\boldsymbol{g}(\boldsymbol{q}) & =\mathbf{0} .
\end{aligned}
$$

They are called d'Alembert-type equations of motion, since the elimination of the constraint forces from the system by premultiplication with the null space matrix is closely related to d'Alembert's principle saying that the virtual work done by constraint forces is zero. Admissible virtual variations in $T_{\boldsymbol{q}} C$ can be expressed as $\delta \boldsymbol{q}=\boldsymbol{P}(\boldsymbol{q}) \cdot \delta \boldsymbol{w}$ with $\delta \boldsymbol{w} \in \mathbb{R}^{n-m}$. With these preliminaries, D'Alembert's principle reads

$$
\delta \boldsymbol{q}^{T} \cdot \boldsymbol{G}^{T}(\boldsymbol{q}) \cdot \boldsymbol{\lambda}=(\boldsymbol{P}(\boldsymbol{q}) \cdot \delta \boldsymbol{w})^{T} \cdot \boldsymbol{G}^{T}(\boldsymbol{q}) \cdot \boldsymbol{\lambda}=0 \quad \forall \delta \boldsymbol{w} \in \mathbb{R}^{n-m} .
$$

Remark 2.2 (Continuous null space matrix) Note that the null space matrix is not unique, a necessary and sufficient condition on $\boldsymbol{P}(\boldsymbol{q})$ is (5). The null space matrix can be found in different ways, either by velocity analysis (i.e. corresponding to (6), the map mapping the independent generalized velocities to the redundant velocities represents a viable null space matrix) or by performing a QR-decomposition of the transposed continuous constraint Jacobian

$$
\boldsymbol{G}^{T}=\boldsymbol{Q} \cdot \boldsymbol{R}=\left[\boldsymbol{Q}_{1}, \boldsymbol{Q}_{2}\right] \cdot\left[\begin{array}{c}
\boldsymbol{R}_{1} \\
\boldsymbol{0}_{(n-m) \times m}
\end{array}\right]
$$

with the nonsingular upper triangular matrix $\boldsymbol{R}_{1} \in \mathbb{R}^{m \times m}$ and the orthogonal matrix $Q \in O(n)$, which can be partitioned into the orthogonal matrices $\boldsymbol{Q}_{1} \in \mathbb{R}^{n \times m}$ and $\boldsymbol{Q}_{2} \in \mathbb{R}^{n \times(n-m)}$. Then $\boldsymbol{P}(\boldsymbol{q})=\boldsymbol{Q}_{2}(\boldsymbol{q})$ serves as null space matrix, which is sometimes called 'natural orthogonal complement' (see [18]). The third way to obtain a continuous null space matrix as the Jacobian of the reparametrization of the constraint manifold is often possible, but the resulting continuous null space matrix can in general not be used to infer a discrete null space matrix. This is due to the fact that the respective discrete values of the generalized coordinates are not available in the present approach. 
Reparametrization in generalized coordinates. For many applications it is possible to find a reparametrization of the constraint manifold $\boldsymbol{F}: U \subseteq \mathbb{R}^{n-m} \rightarrow C$ in terms of independent generalized coordinates $\boldsymbol{u} \in U$. Then the Jacobian $D \boldsymbol{F}(\boldsymbol{u})$ of the coordinate transformation plays the role of a null space matrix. In the sequel, the $(n-m)$-dimensional manifold $U$ will be termed generalized manifold. Since the constraints are fulfilled automatically by the reparametrized configuration variable $\boldsymbol{q}=\boldsymbol{F}(\boldsymbol{u})$, the system is reduced to $n-m$ second order differential equations. The equations of motion of minimal possible dimension for the present mechanical system (which consists of precisely $n-m$ degrees of freedom) then read

$$
D \boldsymbol{F}^{T}(\boldsymbol{u}) \cdot\left[\frac{\partial L(\boldsymbol{q}, \dot{\boldsymbol{q}})}{\partial \boldsymbol{q}}-\frac{d}{d t}\left(\frac{\partial L(\boldsymbol{q}, \dot{\boldsymbol{q}})}{\partial \dot{\boldsymbol{q}}}\right)\right]=\mathbf{0} .
$$

Using (10), one can write

$$
\frac{d}{d t}\left(D \boldsymbol{F}^{T}(\boldsymbol{u}) \cdot \frac{\partial L(\boldsymbol{q}, \dot{\boldsymbol{q}})}{\partial \dot{\boldsymbol{q}}}\right)=\left(D\left(D \boldsymbol{F}^{T}(\boldsymbol{u})\right) \cdot \dot{\boldsymbol{u}}\right) \cdot \frac{\partial L(\boldsymbol{q}, \dot{\boldsymbol{q}})}{\partial \dot{\boldsymbol{q}}}+D \boldsymbol{F}^{T}(\boldsymbol{u}) \cdot \frac{\partial L(\boldsymbol{q}, \dot{\boldsymbol{q}})}{\partial \boldsymbol{q}}
$$

On the other hand, defining a Lagrangian in generalized coordinates $L^{U}: T U \rightarrow \mathbb{R}$ by $L^{U}(\boldsymbol{u}, \dot{\boldsymbol{u}})=L\left(\boldsymbol{F}(\boldsymbol{u}), D \boldsymbol{F}^{T}(\boldsymbol{u}) \cdot \dot{\boldsymbol{u}}\right)$, its partial derivatives read

$$
\begin{aligned}
& \frac{\partial L^{U}(\boldsymbol{u}, \dot{\boldsymbol{u}})}{\partial \boldsymbol{u}}=D \boldsymbol{F}^{T}(\boldsymbol{u}) \cdot \frac{\partial L(\boldsymbol{q}, \dot{\boldsymbol{q}})}{\partial \boldsymbol{q}}+\left(D\left(D \boldsymbol{F}^{T}(\boldsymbol{u})\right) \cdot \dot{\boldsymbol{u}}\right) \cdot \frac{\partial L(\boldsymbol{q}, \dot{\boldsymbol{q}})}{\partial \dot{\boldsymbol{q}}} \\
& \frac{\partial L^{U}(\boldsymbol{u}, \dot{\boldsymbol{u}})}{\partial \dot{\boldsymbol{u}}}=D \boldsymbol{F}^{T}(\boldsymbol{u}) \cdot \frac{\partial L(\boldsymbol{q}, \dot{\boldsymbol{q}})}{\partial \dot{\boldsymbol{q}}}
\end{aligned}
$$

Thus (10) is equivalent to the equations of motion in terms of generalized coordinates

$$
\frac{\partial L^{U}(\boldsymbol{u}, \dot{\boldsymbol{u}})}{\partial \boldsymbol{u}}-\frac{d}{d t}\left(\frac{\partial L^{U}(\boldsymbol{u}, \dot{\boldsymbol{u}})}{\partial \dot{\boldsymbol{u}}}\right)=\mathbf{0} .
$$

Corollary 2.3 The equations of motion (3), (7), (10), and (13) are equivalent, they yield the same motion $q(t)$ in $t \in\left[t_{0}, t_{N}\right]$

Remark 2.4 (Restricted Lagrangian) It is important to note that even though the restricted Lagrangian $L^{C}: T C \rightarrow \mathbb{R}$ can also be written as $L^{C}(\boldsymbol{q}, \dot{\boldsymbol{q}})=L(\boldsymbol{q}, \dot{\boldsymbol{q}})$ with $\boldsymbol{q}=\boldsymbol{F}(\boldsymbol{u})$ and $\dot{\boldsymbol{q}}=D \boldsymbol{F}(\boldsymbol{u}) \cdot \dot{\boldsymbol{u}}$, it is different from $L^{U}$ because the two functions are defined on different domains.

Remark 2.5 (Projections) The premultiplication of the Euler-Lagrange equations by the transposed null space matrix in (7) can be interpreted as a projection onto the cotangent space of the generalized manifold since $\boldsymbol{P}^{T}(\boldsymbol{q}): T_{\boldsymbol{q}}^{*} Q \rightarrow T_{\boldsymbol{u}}^{*} U$. The same holds for the special case in (10) where the Jacobian of the reparametrization serves as a null space matrix. Alternatively, one could think of premultiplying (3) $)_{1}$ by the projection $\boldsymbol{Q}(\boldsymbol{q}): T_{\boldsymbol{q}}^{*} Q \rightarrow \eta\left(T_{\boldsymbol{q}}^{*} C\right)$ where

$$
\eta\left(T_{\boldsymbol{q}}^{*} C\right)=\left\{(\boldsymbol{q}, \boldsymbol{p}) \mid(\boldsymbol{q}, \boldsymbol{p}) \in T_{\boldsymbol{q}}^{*} Q, \boldsymbol{g}(\boldsymbol{q})=\mathbf{0}, \boldsymbol{G}(\boldsymbol{q}) \cdot(\mathbb{F} L(\boldsymbol{p}))^{-1}=\mathbf{0}\right\} \subset T^{*} Q
$$

Even though the constraint forces are eliminated by this projection, the resulting equations of motion are redundant, since they have been projected onto a lower dimensional submanifold. $\eta: T^{*} C \rightarrow T^{*} Q$ is the embedding defined by requiring that the following diagram commutes (see [8] for further details).

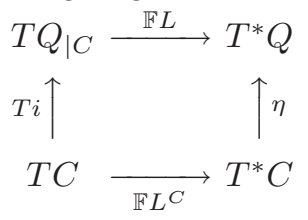

Such a projection can be calculated as

$$
\boldsymbol{Q}=\boldsymbol{I}_{n \times n}-\boldsymbol{G}^{T} \cdot\left[\boldsymbol{G} \cdot \boldsymbol{M}^{-1} \cdot \boldsymbol{G}^{T}\right]^{-1} \boldsymbol{G} \cdot \boldsymbol{M}^{-1},
$$

where $\boldsymbol{I}_{n \times n}$ is the $n \times n$ identity matrix and all quantities are evaluated at $\boldsymbol{q}$. 


\section{Constrained discrete variational dynamics}

Corresponding to the configuration manifold $Q$, the discrete state space is defined by $Q \times Q$ which is locally isomorphic to $T Q$. For a constant time-step $h \in \mathbb{R}$, a path $\boldsymbol{q}:\left[t_{0}, t_{N}\right] \rightarrow Q$ is replaced by a discrete path $\boldsymbol{q}_{d}:\left\{t_{0}, t_{0}+h, \ldots, t_{0}+N h=\right.$ $\left.t_{N}\right\} \rightarrow Q, N \in \mathbb{N}$, where $\boldsymbol{q}_{n}=\boldsymbol{q}_{d}\left(t_{0}+n h\right)$ is viewed as an approximation to $\boldsymbol{q}\left(t_{0}+n h\right)$. Similarly, $\boldsymbol{\lambda}_{n}=\boldsymbol{\lambda}_{d}\left(t_{n}\right)$ approximates the Lagrange multiplier at $t_{n}=t_{0}+n h$.

According to the key idea of variational integrators, the variational principle is discretized rather than the resulting equations of motion. The action integral is approximated in a time interval $\left[t_{n}, t_{n+1}\right]$ using the discrete Lagrangian $L_{d}$ : $Q \times Q \rightarrow \mathbb{R}$ via

$$
L_{d}\left(\boldsymbol{q}_{n}, \boldsymbol{q}_{n+1}\right) \approx \int_{t_{n}}^{t_{n+1}} L(\boldsymbol{q}, \dot{\boldsymbol{q}}) d t .
$$

In this work, the midpoint approximation

$$
L_{d}\left(\boldsymbol{q}_{n}, \boldsymbol{q}_{n+1}\right)=\frac{1}{2 h}\left(\boldsymbol{q}_{n+1}-\boldsymbol{q}_{n}\right)^{T} \cdot \boldsymbol{M} \cdot\left(\boldsymbol{q}_{n+1}-\boldsymbol{q}_{n}\right)-h V\left(\frac{\boldsymbol{q}_{n+1}+\boldsymbol{q}_{n}}{2}\right)
$$

is used. Variation of the discrete action sum

$$
S_{d}=\sum_{n=0}^{N-1} L_{d}\left(\boldsymbol{q}_{n}, \boldsymbol{q}_{n+1}\right)
$$

reads

$$
\delta S_{d}=\delta \boldsymbol{q}_{0}^{T} \cdot D_{1} L_{d}\left(\boldsymbol{q}_{0}, \boldsymbol{q}_{1}\right)+\sum_{n=1}^{N-1} \delta \boldsymbol{q}_{n}^{T} \cdot\left(D_{2} L_{d}\left(\boldsymbol{q}_{n-1}, \boldsymbol{q}_{n}\right)+D_{1} L_{d}\left(\boldsymbol{q}_{n}, \boldsymbol{q}_{n+1}\right)\right)+\delta \boldsymbol{q}_{N}^{T} \cdot D_{2} L_{d}\left(\boldsymbol{q}_{N-1}, \boldsymbol{q}_{N}\right)
$$

Requiring its stationarity for all $\left\{\delta \boldsymbol{q}_{n}\right\}_{n=1}^{N-1}$ and $\delta \boldsymbol{q}_{0}=\delta \boldsymbol{q}_{N}=\mathbf{0}$ yields the discrete (unconstrained) Euler-Lagrange equations

$$
D_{1} L_{d}\left(\boldsymbol{q}_{n}, \boldsymbol{q}_{n+1}\right)+D_{2} L_{d}\left(\boldsymbol{q}_{n-1}, \boldsymbol{q}_{n}\right)=\mathbf{0} .
$$

The integral in $\left[t_{n}, t_{n+1}\right]$ of the scalar product of the constraints and the corresponding Lagrange multiplier is approximated by

$$
\frac{1}{2} \boldsymbol{g}_{d}^{T}\left(\boldsymbol{q}_{n}\right) \cdot \boldsymbol{\lambda}_{n}+\frac{1}{2} \boldsymbol{g}_{d}^{T}\left(\boldsymbol{q}_{n+1}\right) \cdot \boldsymbol{\lambda}_{n+1} \approx \int_{t_{n}}^{t_{n+1}} \boldsymbol{g}^{T}(\boldsymbol{q}) \cdot \boldsymbol{\lambda} d t
$$

whereby $\boldsymbol{g}_{d}^{T}\left(\boldsymbol{q}_{n}\right)=h \boldsymbol{g}^{T}\left(\boldsymbol{q}_{n}\right)$ is used and let $\boldsymbol{G}_{d}^{T}\left(\boldsymbol{q}_{n}\right)=D \boldsymbol{g}_{d}^{T}\left(\boldsymbol{q}_{n}\right)$.

Analogue to Theorem 2.1, the relation between the constrained discrete Lagrangian system on $Q \times Q$ and that corresponding to a discrete Lagrangian restricted to $C \times C$ is stated in the following theorem which has again been taken from [8]. Let $\mathcal{C}_{d}(Q)=\mathcal{C}\left(\left\{t_{0}, t_{0}+h, \ldots, t_{0}+N h=t_{N}\right\}, Q, \boldsymbol{q}_{0}, \boldsymbol{q}_{N}\right)$ denote the space of discrete trajectories satisfying $\boldsymbol{q}_{d}\left(t_{0}\right)=\boldsymbol{q}_{0}$ and $\boldsymbol{q}_{d}\left(t_{N}\right)=\boldsymbol{q}_{N}$ for given $\boldsymbol{q}_{0}, \boldsymbol{q}_{N} \in C$. Let $\mathcal{C}_{d}(C)$ denote the corresponding set of discrete trajectories in $C$ and set $\mathcal{C}_{d}\left(\mathbb{R}^{m}\right)=\mathcal{C}\left(\left\{t_{0}, t_{0}+h, \ldots, t_{0}+(N) h=t_{N}\right\}, \mathbb{R}^{m}\right)$ to be the set of maps $\boldsymbol{\lambda}_{d}:\left\{t_{0}, t_{0}+h, \ldots, t_{0}+(N) h=t_{N}\right\} \rightarrow \mathbb{R}^{m}$ with no boundary conditions.

Theorem 3.1 Suppose that $\mathbf{0}$ is a regular value of the scleronomic holonomic constraints $\boldsymbol{g}: Q \rightarrow \mathbb{R}^{m}$ and set $C=g^{-1}(\mathbf{0}) \subset Q$. Let $L_{d}: Q \times Q \rightarrow \mathbb{R}$ be a discrete Lagrangian and $L_{d}^{C}=L_{d_{\mid C \times C}}$ its restriction to $C \times C$. Then the following statements are equivalent:

(i) $\boldsymbol{q}_{d}=\left\{\boldsymbol{q}_{n}\right\}_{n=0}^{N} \in \mathcal{C}_{d}(C)$ extremizes the discrete action $S_{d}^{C}=S_{d_{\mid C \times C}}$ and hence solves the discrete Euler-Lagrange equations for $L_{d}^{C}$.

(ii) $\left\{\boldsymbol{q}_{n}\right\}_{n=1}^{N-1} \in \mathcal{C}_{d}(C)$ and $\left\{\boldsymbol{\lambda}_{n}\right\}_{n=1}^{N-1} \in \mathcal{C}_{d}\left(\mathbb{R}^{m}\right)$ satisfy the constrained discrete Euler-Lagrange equations

$$
\begin{aligned}
D_{1} L_{d}\left(\boldsymbol{q}_{n}, \boldsymbol{q}_{n+1}\right)+D_{2} L_{d}\left(\boldsymbol{q}_{n-1}, \boldsymbol{q}_{n}\right)-\boldsymbol{G}_{d}^{T}\left(\boldsymbol{q}_{n}\right) \cdot \boldsymbol{\lambda}_{n} & =\mathbf{0}, \\
\boldsymbol{g}\left(\boldsymbol{q}_{n+1}\right) & =\mathbf{0} .
\end{aligned}
$$

(iii) $\left(\boldsymbol{q}_{d}, \boldsymbol{\lambda}_{d}\right) \in \mathcal{C}_{d}\left(Q \times \mathbb{R}^{m}\right)$ extremize $\bar{S}_{d}\left(\boldsymbol{q}_{d}, \boldsymbol{\lambda}_{d}\right)=S_{d}\left(\boldsymbol{q}_{d}\right)-\left\langle\boldsymbol{\lambda}_{d}, \boldsymbol{g}_{d}\left(\boldsymbol{q}_{d}\right)\right\rangle$ and hence, solve the Euler-Lagrange equations for the augmented Lagrangian $\bar{L}_{d}:\left(Q \times \mathbb{R}^{m}\right) \times\left(Q \times \mathbb{R}^{m}\right) \rightarrow \mathbb{R}$ defined by

$$
\bar{L}_{d}\left(\boldsymbol{q}_{n}, \boldsymbol{\lambda}_{n}, \boldsymbol{q}_{n+1}, \boldsymbol{\lambda}_{n+1}\right)=L_{d}\left(\boldsymbol{q}_{n}, \boldsymbol{q}_{n+1}\right)-\frac{1}{2} \boldsymbol{g}_{d}^{T}\left(\boldsymbol{q}_{n}\right) \cdot \boldsymbol{\lambda}_{n}-\frac{1}{2} \boldsymbol{g}_{d}^{T}\left(\boldsymbol{q}_{n+1}\right) \cdot \boldsymbol{\lambda}_{n+1} .
$$


Remark 3.2 (Augmented discrete Lagrangian) The particular choice of the augmented discrete Lagrangian (23) has several consequences that are illustrated by numerical examples in Sect.3.1. First of all, the negative sign in front of the scalar product of constraints and Lagrange multipliers causes the constraint forces $-\boldsymbol{G}^{T}\left(\boldsymbol{q}_{n}\right) \cdot \boldsymbol{\lambda}_{n}$ to have the right orientation. Secondly, including the scalar product of the constraints with the Lagrange multiplier at both time nodes yields contributions of the constraint forces in both discrete Legendre transforms (34), (35). One consequence of that is that the first Lagrange multiplier $\boldsymbol{\lambda}_{0}$ which is computed together with $\boldsymbol{q}_{1}$ using (34) has the correct absolute value. Another consequence is that in the absence of a potential, the discrete Legendre transforms (34), (35) yield conjugate momenta which are consistent with the constraints on momentum level.

Discrete null space method. The reduction of the time-stepping scheme (22) can be accomplished in analogy to the continuous case according to the discrete null space method. This idea has first been introduced in the context of energymomentum conserving integration and applied to he constrained dynamics of mass point systems in [10], then it has been further developed in [14-16] for rigid and flexible multibody systems. In order to eliminate the discrete constraint forces from the equations, a discrete null space matrix fulfilling

$$
\text { range }\left(\boldsymbol{P}\left(\boldsymbol{q}_{n}\right)\right)=\operatorname{null}\left(\boldsymbol{G}_{d}\left(\boldsymbol{q}_{n}\right)\right)
$$

is employed. Analogue to (7), the premultiplication of $(22)_{1}$ by the transposed discrete null space matrix cancels the constraint forces from the system, i.e. the Lagrange multipliers are eliminated from the set of unknowns and the system's dimension is reduced to $n$.

$$
\begin{aligned}
\boldsymbol{P}^{T}\left(\boldsymbol{q}_{n}\right) \cdot\left[D_{2} L_{d}\left(\boldsymbol{q}_{n-1}, \boldsymbol{q}_{n}\right)+D_{1} L_{d}\left(\boldsymbol{q}_{n}, \boldsymbol{q}_{n+1}\right)\right] & =\mathbf{0}, \\
\boldsymbol{g}\left(\boldsymbol{q}_{n+1}\right) & =\mathbf{0} .
\end{aligned}
$$

Proposition 3.3 The d'Alembert-type time-stepping scheme (25) is equivalent to the constrained scheme (22).

Proof. Recapitulating the construction procedure of the d'Alembert-type scheme from the constrained scheme, it is obvious that for given values $\left(\boldsymbol{q}_{n-1}, \boldsymbol{q}_{n}\right)$, a solution $\left(\boldsymbol{q}_{n+1}, \boldsymbol{\lambda}_{n}\right)$ of the constrained scheme (22) is also a solution of the d'Alembert-type scheme (25).

Assume that $\boldsymbol{q}_{n+1}$ solves the d'Alembert-type scheme (25) for given $\left(\boldsymbol{q}_{n-1}, \boldsymbol{q}_{n}\right)$. Note that condition (24) on the discrete null space matrix implies null $\left(\boldsymbol{P}^{T}\left(\boldsymbol{q}_{n}\right)\right)=$ range $\left(\boldsymbol{G}_{d}^{T}\left(\boldsymbol{q}_{n}\right)\right)$ (see e.g. [19]). Together with (25) it follows that

$$
\left[D_{2} L_{d}\left(\boldsymbol{q}_{n-1}, \boldsymbol{q}_{n}\right)+D_{1} L_{d}\left(\boldsymbol{q}_{n}, \boldsymbol{q}_{n+1}\right)\right] \in \operatorname{null}\left(\boldsymbol{P}^{T}\left(\boldsymbol{q}_{n}\right)\right)=\operatorname{range}\left(\boldsymbol{G}_{d}^{T}\left(\boldsymbol{q}_{n}\right)\right) .
$$

Accordingly, there exists a multiplier $\boldsymbol{\lambda}_{n} \in \mathbb{R}^{m}$ such that $\left(\boldsymbol{q}_{n+1}, \boldsymbol{\lambda}_{n}\right)$ solve the constrained scheme (22). An explicit formula to compute $\boldsymbol{\lambda}_{n}$ is given in (38).

Therefore, the d'Alembert-type scheme has the same conservation properties as the constrained scheme. Symplecticity and momentum maps are conserved along a discrete trajectory $\boldsymbol{q}_{d}$ of (25) and the constraints are fulfilled exactly at the time nodes.

Remark 3.4 (Difference to energy-momentum scheme) It is important to note, that the choice to evaluate the constraints and the Lagrange multipliers at the time nodes in (23) causes the evaluation of the constraint Jacobian in (22) at the time nodes. Therefore a discrete null space matrix with the property (24) can simply be found by evaluation of the continuous null space matrix at the time nodes. Acquaintance of the continuous null space matrix for a specific mechanical system always yields an explicit representation of the discrete null space matrix for the variational time-stepping scheme emanating from the discrete variational principle in conjunction with the chosen approximations. This is in contrast to energy-momentum conserving time-stepping schemes based on the concept of discrete derivatives [10,20] or on finite elements in time [21], where the discrete constraint Jacobian $\mathbf{G}\left(\boldsymbol{q}_{n}, \boldsymbol{q}_{n+1}\right)$ depends on both the present and the unknown configuration. As a consequence of the more rare appearance of $\boldsymbol{q}_{n+1}$, the linearization of the variational schemes (22) and (25), necessary to solve the nonlinear algebraic system iteratively, is simpler and less computationally cost-intensive.

Nodal reparametrization. Similar to the continuous case, a reduction of the system to the minimal possible dimension can be accomplished by a local reparametrization of the constraint manifold in the neighborhood of the discrete configuration variable $\boldsymbol{q}_{n} \in C$. At the time nodes, $\boldsymbol{q}_{n}$ is expressed in terms of the discrete generalized coordinates $\boldsymbol{u}_{n} \in U \subseteq \mathbb{R}^{n-m}$, such that the constraints are fulfilled

$$
\boldsymbol{q}_{n}=\boldsymbol{F}_{d}\left(\boldsymbol{u}_{n}, \boldsymbol{q}_{n-1}\right) \text { with } \boldsymbol{g}\left(\boldsymbol{q}_{n}\right)=\boldsymbol{g}\left(\boldsymbol{F}_{d}\left(\boldsymbol{u}_{n}, \boldsymbol{q}_{n-1}\right)\right)=\mathbf{0}
$$


Note that the discrete generalized coordinates $\boldsymbol{u}_{n}$ are incremental variables that describe the evolution of the configuration variable in one time interval only. This avoids the danger of encountering singularities which are often present in absolute reparametrizations. Insertion of the nodal reparametrizations for the configuration (27) into the scheme redundantises $(25)_{2}$. The resulting scheme $n-m$-dimensional scheme

$$
\boldsymbol{P}^{T}\left(\boldsymbol{q}_{n}\right) \cdot\left[D_{2} L_{d}\left(\boldsymbol{q}_{n-1}, \boldsymbol{q}_{n}\right)+D_{1} L_{d}\left(\boldsymbol{q}_{n}, \boldsymbol{F}_{d}\left(\boldsymbol{u}_{n+1}, \boldsymbol{q}_{n}\right)\right)\right]=\mathbf{0}
$$

has to be solved for $\boldsymbol{u}_{n+1}$, then $\boldsymbol{q}_{n+1}$ is obtained by the reparametrization (27). (28) is equivalent to the constrained scheme (22), thus it also has the key properties of exact constraint fulfillment, symplecticity and momentum conservation. While the constrained scheme becomes increasingly ill-conditioned for decreasing time-steps, the condition number of (25), (28) is independent of the time-step.

Starting with the discrete reparametrization $\boldsymbol{q}_{n}=\boldsymbol{F}_{d}\left(\boldsymbol{u}_{n}, \boldsymbol{q}_{n-1}\right)$, it is possible to derive (28) directly in one step. The variation of a redundant configuration variable can be expressed in terms of variations of the discrete generalized coordinates as

$$
\delta \boldsymbol{q}_{n}=\frac{\partial \boldsymbol{F}_{d}}{\partial \boldsymbol{u}_{n}} \cdot \delta \boldsymbol{u}_{n}+\sum_{k=1}^{n-1}\left[\left(\prod_{i=n-1}^{k} \frac{\partial \boldsymbol{F}_{d}}{\partial \boldsymbol{q}_{i}}\right) \cdot \frac{\partial \boldsymbol{F}_{d}}{\partial \boldsymbol{u}_{k}} \cdot \delta \boldsymbol{u}_{k}\right]+\left(\prod_{i=n-1}^{0} \frac{\partial \boldsymbol{F}_{d}}{\partial \boldsymbol{q}_{i}}\right) \cdot \delta \boldsymbol{q}_{0} .
$$

Here, the discrete variational principle (19) requires stationarity for all $\left\{\delta \boldsymbol{u}_{n}\right\}_{n=1}^{N}$ with $\delta \boldsymbol{q}_{0}=\delta \boldsymbol{q}_{N}=\mathbf{0}$. After inserting (29) into (19), the variation $\delta \boldsymbol{u}_{N-1}$ appears only in the last term of the sum in (19) implying $\left(\frac{\partial \boldsymbol{F}_{d}}{\partial \boldsymbol{u}_{N-1}}\right)^{T}$. $\left[D_{2} L_{d}\left(\boldsymbol{q}_{N-2}, \boldsymbol{q}_{N-1}\right)+D_{1} L_{d}\left(\boldsymbol{q}_{N-1}, \boldsymbol{q}_{N}\right)\right]=\mathbf{0}$. Repeating this argument, one arrives at the variational d'Alembert-type scheme with nodal reparametrization

$$
\left(\frac{\partial \boldsymbol{F}_{d}}{\partial \boldsymbol{u}_{n}}\right)^{T} \cdot\left[D_{2} L_{d}\left(\boldsymbol{q}_{n-1}, \boldsymbol{q}_{n}\right)+D_{1} L_{d}\left(\boldsymbol{q}_{n}, \boldsymbol{F}_{d}\left(\boldsymbol{u}_{n+1}, \boldsymbol{q}_{n}\right)\right)\right]=\mathbf{0},
$$

where $\frac{\partial \boldsymbol{F}_{d}}{\partial \boldsymbol{u}_{n}}$ is the discrete null space matrix.

A similar procedure is followed in [9] to derive a reduced variational time-stepping scheme for constrained systems. However, an absolute reparametrization $\boldsymbol{q}_{n}=\boldsymbol{F}\left(\boldsymbol{u}_{n}\right)$ is used there, thus the variational principle is different and the danger of singularities is not excluded.

Corollary 3.5 The discrete time-stepping schemes (22), (25), (28), and (30) are equivalent, they yield the same discrete trajectory $\boldsymbol{q}_{d}$ in $t \in\left[t_{0}, t_{N}\right]$. More specifically, (22), (25), (28), and (30) represent different possibilities to realize the conditions $\boldsymbol{q}_{n} \in C$ and

$$
\delta \boldsymbol{q}_{n}^{T} \cdot\left(D_{1} L_{d}\left(\boldsymbol{q}_{n}, \boldsymbol{q}_{n+1}\right)+D_{2} L_{d}\left(\boldsymbol{q}_{n-1}, \boldsymbol{q}_{n}\right)\right)=0 \quad \forall \delta \boldsymbol{q}_{n} \in T_{\boldsymbol{q}_{n}} C .
$$

Equivalently, one can request $\boldsymbol{q}_{n} \in C$ and

$$
D_{1} L_{d}\left(\boldsymbol{q}_{n}, \boldsymbol{q}_{n+1}\right)+D_{2} L_{d}\left(\boldsymbol{q}_{n-1}, \boldsymbol{q}_{n}\right) \perp T_{\boldsymbol{q}_{n}} C,
$$

whereby the orthogonality condition only makes sense when $D_{1} L_{d}\left(\boldsymbol{q}_{n}, \boldsymbol{q}_{n+1}\right)+D_{2} L_{d}\left(\boldsymbol{q}_{n-1}, \boldsymbol{q}_{n}\right) \in T_{\boldsymbol{q}_{n}}^{*} Q$ is identified with its representing element in $T_{\boldsymbol{q}_{n}} Q$ (using Riez's theorem, see e.g. [22]). Then (32) means

$$
D_{1} L_{d}\left(\boldsymbol{q}_{n}, \boldsymbol{q}_{n+1}\right)+D_{2} L_{d}\left(\boldsymbol{q}_{n-1}, \boldsymbol{q}_{n}\right) \quad \in \operatorname{ann}\left(T_{\boldsymbol{q}_{n}} C\right)=\left\{\boldsymbol{S} \in T_{\boldsymbol{q}_{n}}^{*} Q \mid \boldsymbol{S}_{\mid T_{\boldsymbol{q}_{n}} C}=\mathbf{0}\right\} .
$$

Remark 3.6 (Projections) As mentioned for the continuous case in Remark 2.5, instead of using the discrete null space matrix $\boldsymbol{P}^{T}\left(\boldsymbol{q}_{n}\right): T_{\boldsymbol{q}_{n}}^{*} Q \rightarrow T^{*} U$, one could realize condition (31) or (32) using the projection $\boldsymbol{Q}\left(\boldsymbol{q}_{n}\right): T_{\boldsymbol{q}_{n}}^{*} Q \rightarrow \eta\left(T_{\boldsymbol{q}_{n}}^{*} C\right)$ where $\boldsymbol{Q}\left(\boldsymbol{q}_{n}\right)$ is given by formula (16) and fulfils $\boldsymbol{Q}\left(\boldsymbol{q}_{n}\right) \cdot \boldsymbol{G}_{d}^{T}\left(\boldsymbol{q}_{n}\right)=\mathbf{0}_{n \times m}$. Thereby the constraint forces (including the Lagrange multipliers) are eliminated from the system while the number of equations is not altered. Thus it can not be employed to determine the trajectory since the projection onto the lower dimensional submanifold yields redundant equations. However, this projection will be useful later for certain postprocessing steps of the discrete trajectory where it is important to know conjugate momenta that are consistent with the hidden constraints.

Remark 3.7 ( $\Gamma$-convergence) The $\Gamma$-convergence of discrete action for constrained systems to the corresponding continuum action functional is proven in [23] and the convergence properties of solutions of the discrete Euler-Lagrange equations to stationary points of the continuum problem is studied. This extends the results in [24] to constrained systems. In [23], the convergence result is illustrated with examples of mass point systems and flexible multibody dynamics that make use of the discrete null space method described in detail here in Sects. 3.1, 4, 5, and 6.1. 
Constrained discrete legendre transform. So far, the derivation of variational time-stepping schemes for constrained systems is based solely on the discrete path of configurations, i.e. the discrete trajectory $\boldsymbol{q}_{d} \in C$. Thereby, the discrete Lagrangian (17) involves a finite difference approximation for the velocity in the kinetic energy and an evaluation of the potential energy at some midpoint. Such approximated velocities do not fulfill the temporal differentiated form of the constraints, the so-called hidden constraints. However, information on the systems evolution on velocity or momentum level might be of interest. This can be obtained using a discrete constrained Legendre transform.

Based on the augmented discrete Lagrangian (23), the constrained discrete Legendre transforms $\mathbb{F}^{c^{-}} L_{d}: Q \times Q \rightarrow T_{\boldsymbol{q}_{n}}^{*} Q$ and $\mathbb{F}^{c^{+}} L_{d}: Q \times Q \rightarrow T_{\boldsymbol{q}_{n}}^{*} Q \mathrm{read}$

$$
\begin{aligned}
\mathbb{F}^{c^{-}} L_{d} & :\left(\boldsymbol{q}_{n}, \boldsymbol{q}_{n+1}\right) \mapsto\left(\boldsymbol{q}_{n}, \boldsymbol{p}_{n}^{-}\right) \\
\boldsymbol{p}_{n}^{-} & =-D_{1} L_{d}\left(\boldsymbol{q}_{n}, \boldsymbol{q}_{n+1}\right)+\frac{1}{2} \boldsymbol{G}_{d}^{T}\left(\boldsymbol{q}_{n}\right) \cdot \boldsymbol{\lambda}_{n}, \\
\mathbb{F}^{c^{+}} L_{d} & :\left(\boldsymbol{q}_{n-1}, \boldsymbol{q}_{n}\right) \mapsto\left(\boldsymbol{q}_{n}, \boldsymbol{p}_{n}^{+}\right), \\
\boldsymbol{p}_{n}^{+} & =D_{2} L_{d}\left(\boldsymbol{q}_{n-1}, \boldsymbol{q}_{n}\right)-\frac{1}{2} \boldsymbol{G}_{d}^{T}\left(\boldsymbol{q}_{n}\right) \cdot \boldsymbol{\lambda}_{n} .
\end{aligned}
$$

With (34), (35), the constrained time-stepping scheme $(22)_{1}$ can be interpreted as enforcing the matching of momenta $\boldsymbol{p}_{n}^{+}-\boldsymbol{p}_{n}^{-}=\mathbf{0}$ such that along the discrete trajectory, there is a unique momentum at each time node $n$ which can be denoted by $\boldsymbol{p}_{n}$. When the discrete null space method is used, the Lagrange multipliers are not at hand as an output of the simulation. They can be recovered easily using the $n \times m$ matrix

$$
\boldsymbol{R}_{d}\left(\boldsymbol{q}_{n}\right)=\boldsymbol{G}_{d}^{T}\left(\boldsymbol{q}_{n}\right) \cdot\left(\boldsymbol{G}_{d}\left(\boldsymbol{q}_{n}\right) \cdot \boldsymbol{G}_{d}^{T}\left(\boldsymbol{q}_{n}\right)\right)^{-1}
$$

which obviously fulfills

$$
\boldsymbol{G}_{d}\left(\boldsymbol{q}_{n}\right) \cdot \boldsymbol{R}_{d}\left(\boldsymbol{q}_{n}\right)=\boldsymbol{I}_{m \times m},
$$

where $I_{m \times m}$ denotes the $m$-dimensional identity matrix. Then the Lagrange multipliers can be recovered by premultiplying $(22)_{1}$ by $\boldsymbol{R}^{T}\left(\boldsymbol{q}_{n}\right)$ and accounting for (37). In particular, this yields

$$
\boldsymbol{\lambda}_{n}=\boldsymbol{R}_{d}^{T}\left(\boldsymbol{q}_{n}\right) \cdot\left[D_{1} L_{d}\left(\boldsymbol{q}_{n}, \boldsymbol{q}_{n+1}\right)+D_{2} L_{d}\left(\boldsymbol{q}_{n-1}, \boldsymbol{q}_{n}\right)\right],
$$

whereupon the constrained Legendre transforms can be used. On the other hand, if no information on the constraint forces is needed, one can avoid to recover the Lagrange multipliers and use the projected discrete Legendre transforms ${ }^{Q} \mathbb{F}^{c^{-}} L_{d}:$ $Q \times Q \rightarrow \eta\left(T_{\boldsymbol{q}_{n}}^{*} C\right)$ and ${ }^{Q} \mathbb{F}^{c^{+}} L_{d}: Q \times Q \rightarrow \eta\left(T_{\boldsymbol{q}_{n}}^{*} C\right)$ reading

$$
\begin{aligned}
& { }^{Q} \boldsymbol{p}_{n}^{-}=-\boldsymbol{Q}\left(\boldsymbol{q}_{n}\right) \cdot D_{1} L_{d}\left(\boldsymbol{q}_{n}, \boldsymbol{q}_{n+1}\right), \\
& { }^{Q} \boldsymbol{p}_{n}^{+}=\boldsymbol{Q}\left(\boldsymbol{q}_{n}\right) \cdot D_{2} L_{d}\left(\boldsymbol{q}_{n-1}, \boldsymbol{q}_{n}\right),
\end{aligned}
$$

where $\boldsymbol{Q}\left(\boldsymbol{q}_{n}\right)$ is given by formula (16) and fulfills $\boldsymbol{Q}\left(\boldsymbol{q}_{n}\right) \cdot \boldsymbol{G}_{d}^{T}\left(\boldsymbol{q}_{n}\right)=\mathbf{0}_{n \times m}$. Both projected discrete Legendre transforms yield the same momentum vector denoted by ${ }^{Q} \boldsymbol{p}_{n}$. Note that for the constrained discrete Legendre transforms and for the projected discrete Legendre transforms, the output is an $n$-dimensional momentum vector. In the projected case, it lies in the $(n-m)$-dimensional submanifold $\eta\left(T_{\boldsymbol{q}_{n}}^{*} C\right)$. Yet another possibility is to compute an $(n-m)$-dimensional momentum vector by projecting with the discrete null space matrix. The reduced discrete Legendre transforms ${ }^{P} \mathbb{F}^{c^{-}} L_{d}: Q \times Q \rightarrow T^{*} U$ and ${ }^{P} \mathbb{F}^{c^{+}} L_{d}: Q \times Q \rightarrow T^{*} U$ are given by

$$
\begin{gathered}
{ }^{P} \boldsymbol{p}_{n}^{-}=-\boldsymbol{P}^{T}\left(\boldsymbol{q}_{n}\right) \cdot D_{1} L_{d}\left(\boldsymbol{q}_{n}, \boldsymbol{q}_{n+1}\right), \\
{ }^{P} \boldsymbol{p}_{n}^{+}=\boldsymbol{P}^{T}\left(\boldsymbol{q}_{n}\right) \cdot D_{2} L_{d}\left(\boldsymbol{q}_{n-1}, \boldsymbol{q}_{n}\right) .
\end{gathered}
$$

Hidden constraints. The temporary differentiated form of the configuration constraints yields the so-called secondary or hidden constraints, which have been introduced already without mentioning in (2) and (14)

$$
\begin{aligned}
& \boldsymbol{f}(\boldsymbol{q}, \dot{\boldsymbol{q}})=\frac{d \boldsymbol{g}(\boldsymbol{q})}{d t}=\boldsymbol{G}(\boldsymbol{q}) \cdot \dot{\boldsymbol{q}}=\mathbf{0} \\
& \boldsymbol{h}(\boldsymbol{q}, \boldsymbol{p})=\frac{d \boldsymbol{g}(\boldsymbol{q})}{d t}=\boldsymbol{G}(\boldsymbol{q}) \cdot(\mathbb{F} L(\boldsymbol{p}))^{-1}=\mathbf{0}
\end{aligned}
$$


Similar to (4), in a continuous augmented Lagrangian they could be included as follows

$$
\bar{L}(\boldsymbol{q}, \boldsymbol{\lambda}, \dot{\boldsymbol{q}}, \dot{\boldsymbol{\lambda}}, \boldsymbol{\mu}, \dot{\boldsymbol{\mu}})=L(\boldsymbol{q}, \dot{\boldsymbol{q}})-\boldsymbol{g}^{T}(\boldsymbol{q}) \cdot \boldsymbol{\lambda}-\boldsymbol{f}^{T}(\boldsymbol{q}, \dot{\boldsymbol{q}}) \cdot \boldsymbol{\mu} .
$$

Their discrete form could be enforced during the simulation by inclusion of discrete versions in the augmented discrete Lagrangian. However, the investigation of several numerical examples dealing with the energy-momentum conserving integration of point mass systems, rigid bodies and geometrically exact beams (e.g. in [25], [26]) has brought forward that the incorporation of the temporally differentiated form of the constraints has not lead to crucial advantages (besides the fulfillment of the secondary constraints themselves). This fact is also reported in [27] and references therein. The discrete trajectory has not been influenced considerably by their fulfillment. Thus, their inclusion in the trajectory based approach of variational integrators seems an exaggeration - besides, they are fulfilled to some extent by the discrete trajectory. On velocity level, one possibility is to write the discrete hidden constraints in the form

$$
\boldsymbol{f}_{d}\left(\boldsymbol{q}_{n}, \boldsymbol{q}_{n+1}\right)=\boldsymbol{G}\left(\frac{\boldsymbol{q}_{n+1}+\boldsymbol{q}_{n}}{2}\right) \cdot \frac{\boldsymbol{q}_{n+1}-\boldsymbol{q}_{n}}{h}=\mathbf{0} .
$$

In the example of the mathematical pendulum where the constraint manifold is an equicurved circle, they are fulfilled exactly in presence and in absence of the potential energy. However, for general constraint manifolds, they need not be fulfilled. For Lagrangians of the form $L(\boldsymbol{q}, \dot{\boldsymbol{q}})=\frac{1}{2} \dot{\boldsymbol{q}}^{T} \cdot \boldsymbol{M} \cdot \dot{\boldsymbol{q}}-V(\boldsymbol{q})$, where the conjugate momenta computed by the continuous Legendre transform are given by $\boldsymbol{p}=\boldsymbol{M} \cdot \dot{\boldsymbol{q}}$, it can be inferred from (43) $)_{2}$ that the discrete hidden constraints on momentum level read

$$
\boldsymbol{h}_{d}\left(\boldsymbol{q}_{n}, \boldsymbol{p}_{n}\right)=\boldsymbol{G}\left(\boldsymbol{q}_{n}\right) \cdot \boldsymbol{M}^{-1} \cdot \boldsymbol{p}_{n}=\mathbf{0} .
$$

Of course, $\boldsymbol{h}_{d}\left(\boldsymbol{q}_{n},{ }^{Q} \boldsymbol{p}_{n}\right)=\mathbf{0}$ holds exactly, since null $\left(\boldsymbol{G}\left(\boldsymbol{q}_{n}\right)\right)=T_{\boldsymbol{q}_{n}} C$ and elements in $\eta\left(T_{\boldsymbol{q}_{n}}^{*} C\right)$ can be identified with their representing element in $T_{\boldsymbol{q}_{n}} C$ (using Riez's theorem, see e.g. [22]). To date, experience shows that $\boldsymbol{h}_{d}\left(\boldsymbol{q}_{n}, \boldsymbol{p}_{n}\right)=\mathbf{0}$ is fulfilled in the absence of a potential but not when a potential is present (see Sect. 3.1).

Initialization of the simulation. Another reason for which a discrete constrained Legendre transform is required is the initialization of the simulation. To simulate the motion of a constrained dynamical system with one of the equivalent timestepping schemes, it is necessary to specify an initial configuration $\boldsymbol{q}_{0} \in C$ and a second configuration $\boldsymbol{q}_{1} \in C$. Together with the time-step $h$, their difference quotient represents the velocity of the system in the first time interval $[0, h]$. For non-trivial mechanical systems, it might be difficult or even impossible to come up with a reasonable $\boldsymbol{q}_{1}$ that fulfills the constraints. Since the purpose of numerical time-integration schemes is to approximate a continuous trajectory, it seems appropriate to initialize the simulation by prescribing $\boldsymbol{q}(0) \in C$ and $\dot{\boldsymbol{q}}(0) \in T_{\boldsymbol{q}(0)} C$. The latter can e.g. be found by choosing generalized velocities $\boldsymbol{\nu}(0)$ and employing formula (6). Obviously, an Euler step $\boldsymbol{q}_{1}=h \dot{\boldsymbol{q}}(0)$ does not yield a second configuration that fulfills the constraints. On the other hand, consistent initial momenta can be calculated via the continuous Legendre transform $\boldsymbol{p}(0)=\frac{\partial L}{\partial \dot{\boldsymbol{q}}} \in T_{\boldsymbol{q}(0)}^{*} C$. Finally, setting $\boldsymbol{p}_{0}^{-}=\boldsymbol{p}(0)$, one can solve (34) together with $\boldsymbol{g}\left(\boldsymbol{q}_{1}\right)=\mathbf{0}$ for $\boldsymbol{q}_{1} \in C$ and $\boldsymbol{\lambda}_{0}$. Alternatively, using the discrete null space method, the reduced continuous momenta are given by ${ }^{P} \boldsymbol{p}_{0}^{-}=\boldsymbol{P}^{T}(\boldsymbol{q}(0)) \cdot \boldsymbol{p}(0)$. After inserting ${ }^{P} \boldsymbol{p}_{0}^{-}$and $\boldsymbol{q}_{1}=\boldsymbol{F}_{d}\left(\boldsymbol{u}_{1}, \boldsymbol{q}(0)\right)$ into (41), the latter can be solved for $\boldsymbol{u}_{1}$, then $\boldsymbol{q}_{1}$ follows by nodal reparametrization. In this case, the corresponding Lagrange multiplier can be recovered as

$$
\boldsymbol{\lambda}_{0}=2 \boldsymbol{R}_{d}^{T}(\boldsymbol{q}(0)) \cdot\left[\boldsymbol{p}(0)+D_{1} L_{d}\left(\boldsymbol{q}(0), \boldsymbol{q}_{1}\right)\right] .
$$

Remark 3.8 (Unconstrained systems) For unconstrained systems, one can simply choose $\boldsymbol{q}_{1} \in Q$. Nevertheless, one has to keep in mind that the difference quotient $\frac{q_{1}-q_{0}}{h}$ determines the initial kinetic energy of the system. Also here, one can use the continuous initial state to compute $\boldsymbol{q}_{1}$ from $\boldsymbol{p}_{n}^{-}=-D_{1} L_{d}\left(\boldsymbol{q}_{n}, \boldsymbol{q}_{n+1}\right)$. Only when no potential is present and the motion is rectilinear, an Euler steps coincides with the calculation of $\boldsymbol{q}_{1}$ from the discrete Legendre transform.

\subsection{Numerical example: mathematical pendulum}

As an easy but illustrative example, a two-dimensional $(n=2)$ mathematical pendulum with mass $M=1$, yielding the $2 \times 2$ mass matrix $M=M \boldsymbol{I}_{2 \times 2}$, and rod length $l=1$ is studied. The configuration space is $Q=\mathbb{R}^{2}$ and the constraint manifold is $C=S_{l}^{1}$. First of all, the gravitation is set to zero such that the mass point moves on the unit circle with constant angular velocity. The initial position is $\boldsymbol{q}(0)=[1,0]^{T}$ and the initial generalized velocity is $\nu(0)=1$, thus the total energy of the system is 0.5 . The $m=1$ constraint function, the constraint Jacobian and the null space matrix read

$$
g(\boldsymbol{q})=\frac{1}{2}\left(\boldsymbol{q}^{T} \cdot \boldsymbol{q}-l^{2}\right)=0, \quad \boldsymbol{G}(\boldsymbol{q})=\boldsymbol{q}^{T}, \quad \boldsymbol{P}(\boldsymbol{q})=\left[\begin{array}{c}
-q_{y} \\
q_{x}
\end{array}\right] .
$$




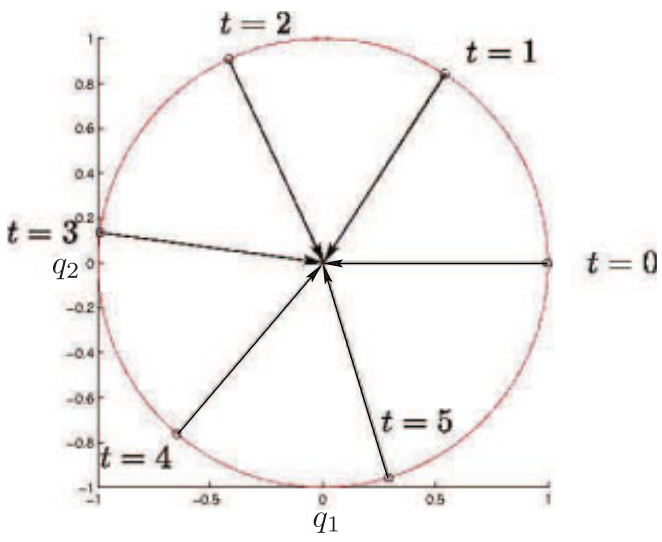

Fig. 1 (online colour at: www.zamm-journal.org) Mass point on unit circle: snapshots of the motion and constraint forces $\left(h=10^{-1}\right)$.

During the time stepping scheme, they are evaluated at $\boldsymbol{q}_{n}$. In the time interval $\left[t_{n}, t_{n+1}\right]$, the mass points rotates about the out of plane axis by the angle $u_{n+1} \in \mathbb{R}$, therefore the discrete reparametrization is given by

$$
\boldsymbol{q}_{n+1}=\boldsymbol{F}_{d}\left(\boldsymbol{u}_{n+1}, \boldsymbol{q}_{n}\right)=\left[\begin{array}{cc}
\cos \left(u_{n+1}\right) & -\sin \left(u_{n+1}\right) \\
\sin \left(u_{n+1}\right) & \cos \left(u_{n+1}\right)
\end{array}\right] \cdot \boldsymbol{q}_{n} .
$$

Using the constrained time-stepping scheme (22), $n+m=3$ equations have to be solved for $\boldsymbol{q}_{n+1}$ and $\lambda_{n}$ while the number of equations is reduced to one if the discrete null space method with nodal reparametrization (28) is used. Fig. 1 shows configurations of the motion and the corresponding constraint forces $\boldsymbol{G}^{T}\left(\boldsymbol{q}_{n}\right) \cdot \boldsymbol{\lambda}_{n}$ that point towards the center of the unit circle.

The diagram on the left in Fig. 2 shows the evolution of the discrete energy $E_{d}$ in the upper graph, calculated in terms of subsequent configurations as

$$
E_{d}\left(\boldsymbol{q}_{n}, \boldsymbol{q}_{n+1}\right)=\frac{1}{2}\left(\frac{\boldsymbol{q}_{n+1}-\boldsymbol{q}_{n}}{h}\right)^{T} \cdot \boldsymbol{M} \cdot\left(\frac{\boldsymbol{q}_{n+1}-\boldsymbol{q}_{n}}{h}\right)
$$

and the lower graph depicts the discrete Hamiltonian $H_{d}$ in terms of the momenta as

$$
H_{d}\left(\boldsymbol{q}_{n}, \boldsymbol{p}_{n}\right)=\frac{1}{2} \boldsymbol{p}_{n}^{T} \cdot \boldsymbol{M}^{-1} \cdot \boldsymbol{p}_{n} .
$$

Note that the expression for the energy in terms of the momenta exactly preserves the initial energy while that in terms of velocity preserves a value which is slightly different, see Fig. 2. This indicates that in the presence of constraints, postprocessing steps should involve the momenta obtained by the discrete Legendre transform rather than the velocities.

Furthermore, one can see in Fig. 1 that besides pointing into the correct direction, the constraint forces do all have the same value $\lambda_{n}=-1.00251257867$, in particular, $\lambda_{0}$ makes no exception. This is a consequence of the discrete Legendre transform (34) being based in the augmented discrete Lagrangian (23) (see also Remark 3.2).
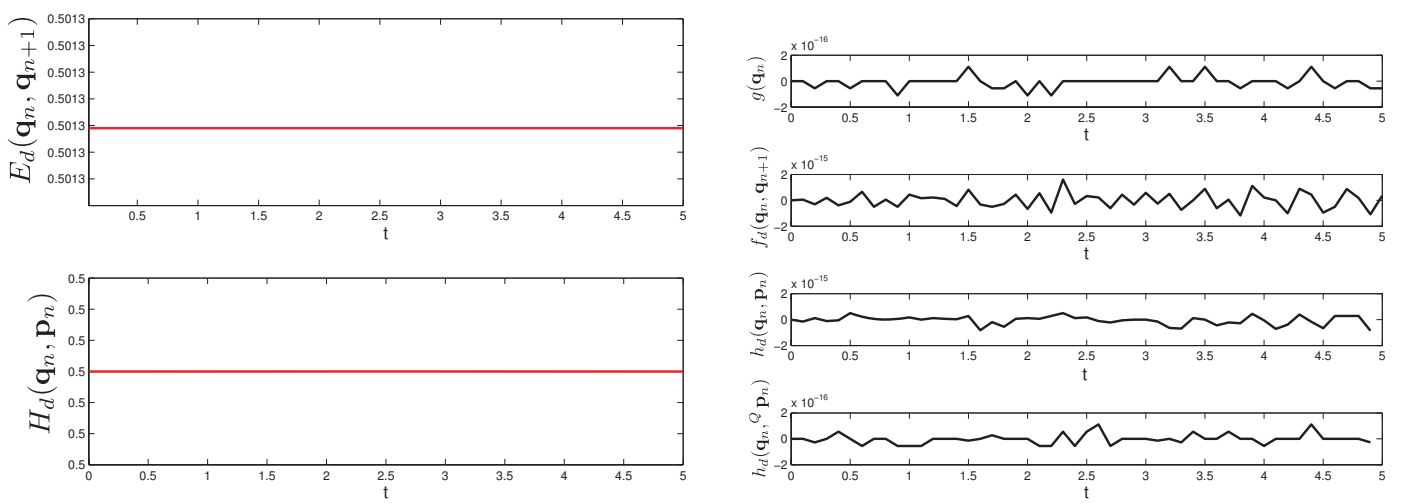

Fig. 2 (online colour at: www.zamm-journal.org) Mass point on unit circle: total energy and fulfillment of the constraints on configuration level, on velocity level, and on momentum level $\left(h=10^{-1}\right)$. 

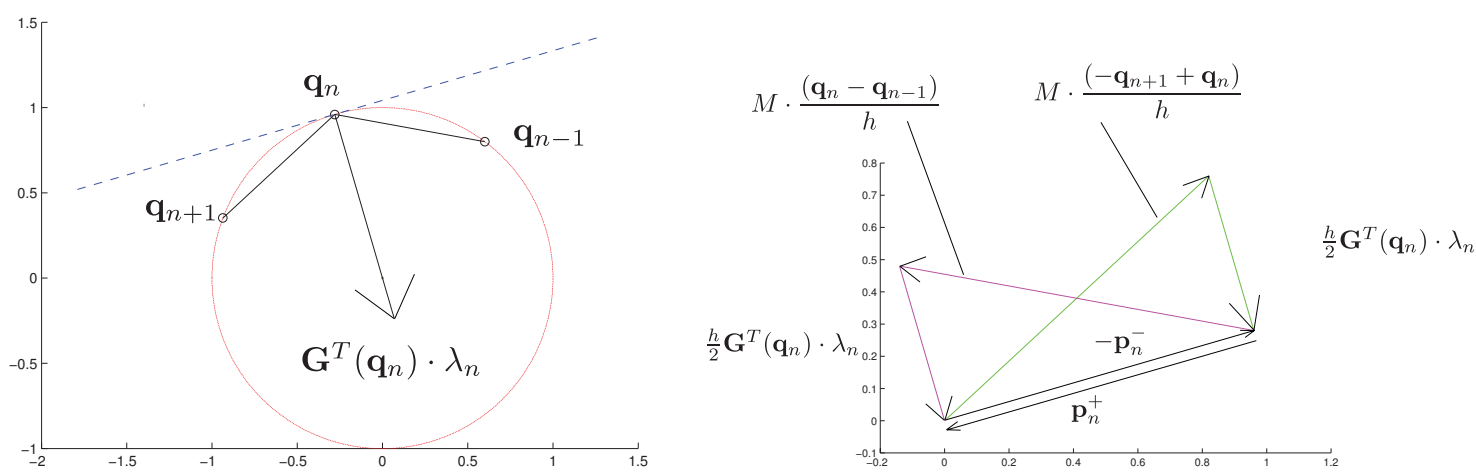

Fig. 3 (online colour at: www.zamm-journal.org) Mass point on unit circle: illustration of the discrete Legendre transform and the matching of momenta $\left(h=8 \cdot 10^{-1}\right)$.
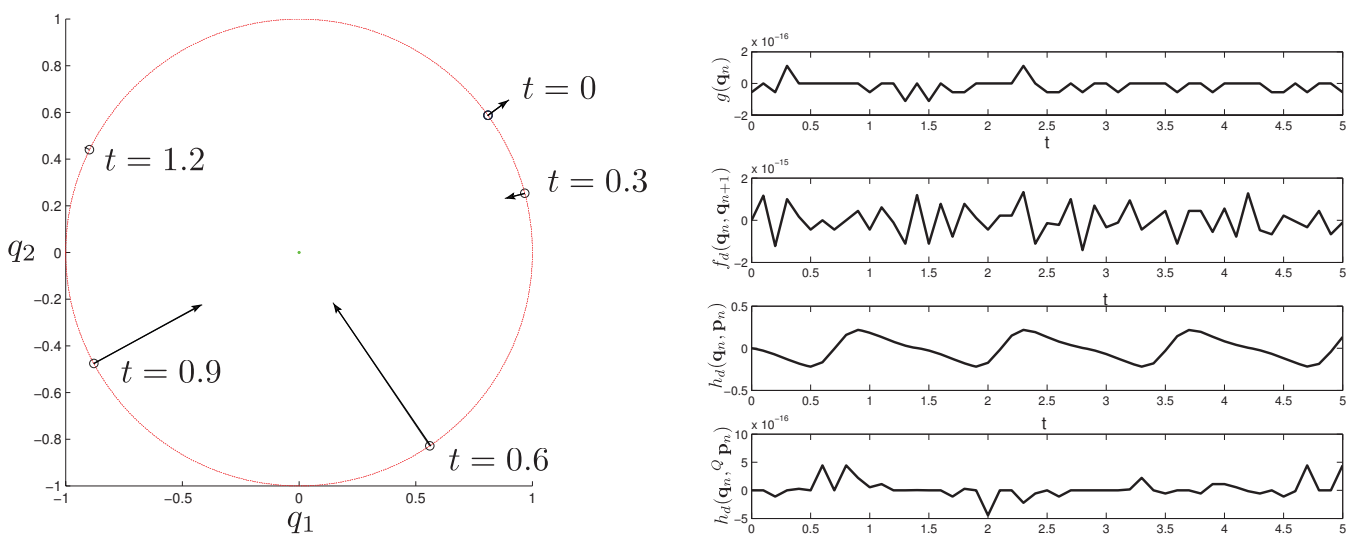

Fig. 4 (online colour at: www.zamm-journal.org) Pendulum: snapshots of the motion and constraint forces and fulfillment of constraints on configuration level, on velocity level, and on momentum level $\left(h=10^{-1}\right)$.

The right hand diagram in Fig. 2 reveals that the primary as well as the secondary constraints (45), (46) are fulfilled exactly in the absence of a potential.

This is no surprise since one can observe from Fig. 3 that velocities are tangential at the midpoints and momenta are tangential to the constraint manifold at the configurations themselves. The results obtained using the constrained scheme are indistinguishable from those using the d'Alembert type scheme with nodal reparametrization.

Secondly, the motion is influenced by the potential $V(\boldsymbol{q})=(\boldsymbol{M} \cdot \boldsymbol{g})^{T} \cdot \boldsymbol{q}$ whereby $\boldsymbol{g}=[0,-9.81]^{T}$. The pendulum is released with no initial velocity from the position $\boldsymbol{q}(0)=[1,0]^{T}$. Fig. 4 shows that the constraint forces again point towards the pendulum's suspension point and their absolute value varies according to the actual configuration.

The energies

$$
E_{d}\left(\boldsymbol{q}_{n}, \boldsymbol{q}_{n+1}\right)=\frac{1}{2}\left(\frac{\boldsymbol{q}_{n+1}-\boldsymbol{q}_{n}}{h}\right)^{T} \cdot \boldsymbol{M} \cdot\left(\frac{\boldsymbol{q}_{n+1}-\boldsymbol{q}_{n}}{h}\right)+V\left(\frac{\boldsymbol{q}_{n+1}+\boldsymbol{q}_{n}}{2}\right)
$$

depicted in the upper graph and

$$
H_{d}\left(\boldsymbol{q}_{n}, \boldsymbol{p}_{n}\right)=\frac{1}{2} \boldsymbol{p}_{n}^{T} \cdot \boldsymbol{M}^{-1} \cdot \boldsymbol{p}_{n}+V\left(\boldsymbol{q}_{n}\right)
$$

depicted in the lower graph of Fig. 5 on the left look very much alike, however, the plot of the total energy on a finer scale on the right reveals that $H_{d}$ oscillates with smaller amplitude than $E_{d}$ and that deviations occur with a different sign.

In the presence of a potential, the distances between two subsequent configurations are not equal as can be observed from Fig. 4 and 6. It is observable from these pictures that $\boldsymbol{q}_{n+1}-\boldsymbol{q}_{n}$ is always tangential to the constraint manifold at the midpoint wherefore the constraints on velocity level are fulfilled exactly (see Fig. 4). However, the momentum vector $\boldsymbol{p}_{n}$ is not in the direction of the tangent (see Fig. 6), thus it is not in the null space of the constraint Jacobian and the hidden constraints are not fulfilled. Of course, the projected momenta fulfill them. Despite being not exactly equal, the plots of the evolution of total energy being computed from $\boldsymbol{p}_{n}$ and ${ }^{Q} \boldsymbol{p}_{n}$ are indistinguishable, wherefore only one of them is shown in Fig. 5. 

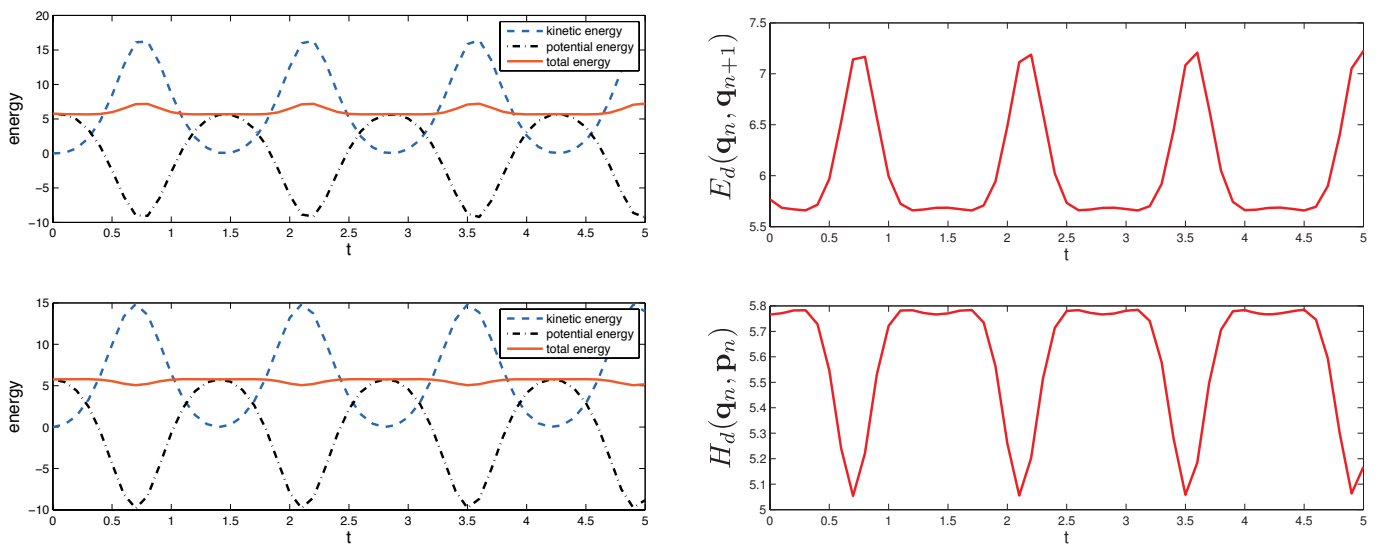

Fig. 5 (online colour at: www.zamm-journal.org) Pendulum: energy in terms of $\left(\boldsymbol{q}_{n}, \boldsymbol{q}_{n+1}\right)$ and $\left(\boldsymbol{q}_{n}, \boldsymbol{p}_{n}\right)\left(h=10^{-1}\right)$.
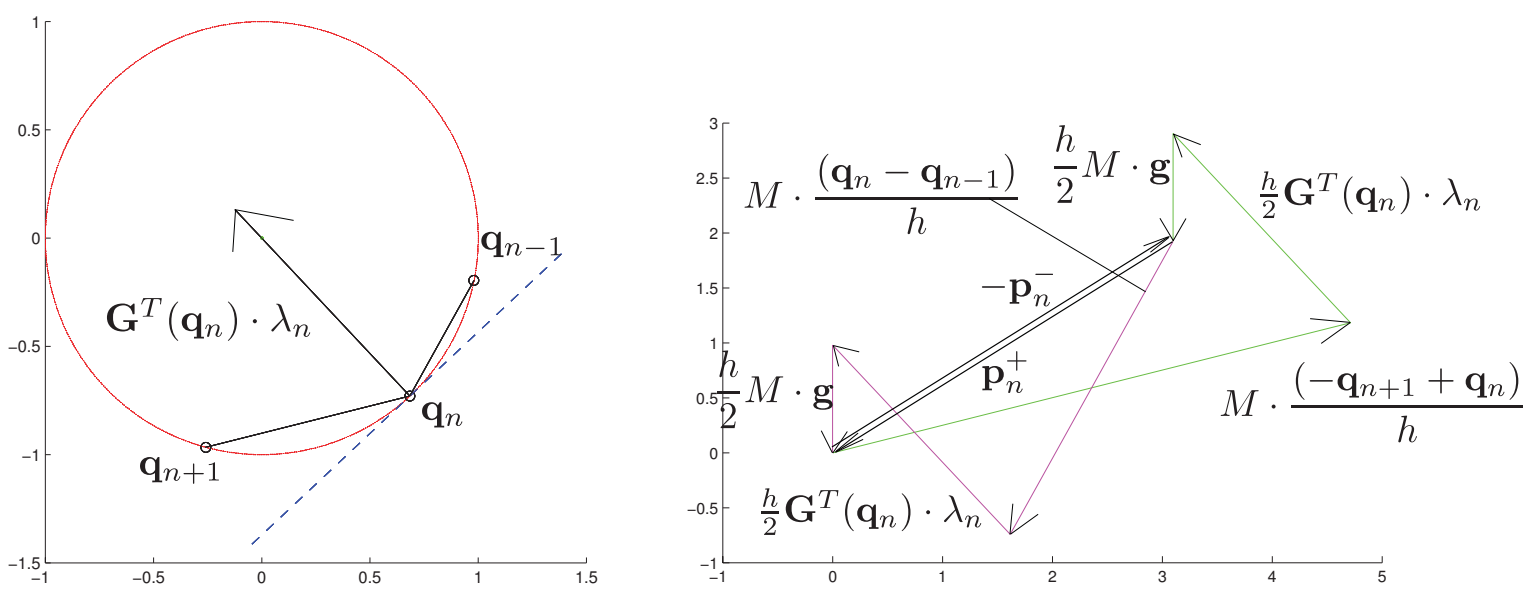

Fig. 6 (online colour at: www.zamm-journal.org) Pendulum: illustration of the discrete Legendre transform and the matching of momenta.

\section{Rigid body dynamics}

This work makes use of a constrained formulation of rigid body dynamics [28], that directly fits in the framework of DAEs. It circumvents the need to deal with rotational parameters, angular velocities and accelerations in the Lagrangian. This formulation is explained in detail in $[14,15]$ where it is also shown that the reduced equations of motion $(7)_{1}$ represent the well-known Newton-Euler equations for the rigid body dynamics. The treatment of rigid bodies as structural elements relies on the kinematic assumptions is illustrated in Fig. 7 (see [29]).

The fact that the placement of a material point in the body's configuration $\boldsymbol{X}=X_{I} \boldsymbol{d}_{I} \in \mathcal{B} \subset \mathbb{R}^{3}$ relative to an orthonormal basis $\left\{\boldsymbol{e}_{I}\right\}$ fixed in space can be described as

$$
\boldsymbol{x}(\boldsymbol{X}, t)=\varphi(t)+X_{I} d_{I}(t)
$$

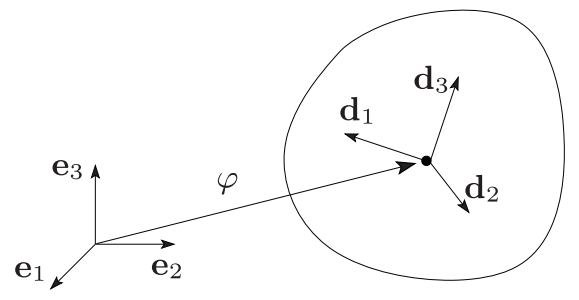

Fig. 7 (online colour at: www.zamm-journal.org) Configuration of a rigid body with respect to an orthonormal frame $\left\{\boldsymbol{e}_{I}\right\}$ fixed in space. 
is used. Here $X_{I} \in \mathbb{R}, I=1,2,3$ represent coordinates in the body-fixed director triad $\left\{\boldsymbol{d}_{I}\right\}$. The time-dependent configuration variable of a rigid body

$$
\boldsymbol{q}(t)=\left[\begin{array}{l}
\boldsymbol{\varphi}(t) \\
\boldsymbol{d}_{1}(t) \\
\boldsymbol{d}_{2}(t) \\
\boldsymbol{d}_{3}(t)
\end{array}\right] \in \mathbb{R}^{12}
$$

consists of the placement of the center of mass $\varphi \in \mathbb{R}^{3}$ and the directors $\boldsymbol{d}_{I} \in \mathbb{R}^{3}, I=1,2,3$ representing the orientation of the rigid body. Due to the body's rigidity, the directors are constrained to stay orthonormal during the motion. Thus one works with the embedding of the constraint manifold $C=\mathbb{R}^{3} \times S O(3)$ into the configuration manifold $Q=\mathbb{R}^{12}$. These orthonormality conditions pertaining to the kinematic assumptions of the underlying theory are termed internal constraints. There are $m_{\mathrm{int}}=6$ independent internal constraints for the rigid body with associated constraint functions

$$
\boldsymbol{g}_{\mathrm{int}}(\boldsymbol{q})=\left[\begin{array}{c}
\frac{1}{2}\left[\boldsymbol{d}_{1}^{T} \cdot \boldsymbol{d}_{1}-1\right] \\
\frac{1}{2}\left[\boldsymbol{d}_{2}^{T} \cdot \boldsymbol{d}_{2}-1\right] \\
\frac{1}{2}\left[\boldsymbol{d}_{3}^{T} \cdot \boldsymbol{d}_{3}-1\right] \\
\boldsymbol{d}_{1}^{T} \cdot \boldsymbol{d}_{2} \\
\boldsymbol{d}_{1}^{T} \cdot \boldsymbol{d}_{3}
\end{array}\right]
$$

For simplicity, it is assumed that the axes of the body frame, i.e. the directors, coincide with the principal axes of inertia of the rigid body. Then the body's Euler tensor with respect to the center of mass can be related to the inertia tensor $\boldsymbol{J}$ via

$$
\boldsymbol{E}=\frac{1}{2}(\operatorname{tr} \boldsymbol{J}) \boldsymbol{I}-\boldsymbol{J}
$$

where $I$ denotes the $3 \times 3$ identity matrix. The principal values of the Euler tensor $E_{I}$ together with the body's total mass $M_{\varphi}$ build the rigid body's constant symmetric positive definite mass matrix

$$
\boldsymbol{M}=\left[\begin{array}{cccc}
M_{\varphi} \boldsymbol{I} & \mathbf{0} & \mathbf{0} & \mathbf{0} \\
\mathbf{0} & E_{1} \boldsymbol{I} & \mathbf{0} & \mathbf{0} \\
\mathbf{0} & \mathbf{0} & E_{2} \boldsymbol{I} & \mathbf{0} \\
\mathbf{0} & \mathbf{0} & \mathbf{0} & E_{3} \boldsymbol{I}
\end{array}\right]
$$

where 0 denotes the $3 \times 3$ zero matrix. This description of rigid body dynamics has been expatiated in [14] where also the null space matrix

$$
\boldsymbol{P}_{\mathrm{int}}(\boldsymbol{q})=\left[\begin{array}{cc}
\boldsymbol{I} & \mathbf{0} \\
\mathbf{0} & -\widehat{\boldsymbol{d}_{1}} \\
\mathbf{0} & -\widehat{\boldsymbol{d}_{2}} \\
\mathbf{0} & -\widehat{\boldsymbol{d}_{3}}
\end{array}\right]
$$

corresponding to the constraints (56) has been derived. Here $\widehat{\boldsymbol{a}}$ denotes the skew-symmetric $3 \times 3$ matrix with corresponding axial vector $\boldsymbol{a} \in \mathbb{R}^{3}$. The derivation of the null space matrix in (59) makes use of (6) and the fact that the independent generalized velocities of a rigid body are the translational velocity $\dot{\varphi} \in \mathbb{R}^{3}$ and the angular velocity $\boldsymbol{\omega} \in \mathbb{R}^{3}$. They can be comprised into the twist of the rigid body

$$
\boldsymbol{t}=\left[\begin{array}{c}
\dot{\varphi} \\
\omega
\end{array}\right]
$$

whereupon (6) yields the director velocities $\dot{\boldsymbol{d}}_{I}=\boldsymbol{\omega} \times \boldsymbol{d}_{I}$ for $I=1,2,3$. When the nodal reparametrization of unknowns is applied, the configuration of the free rigid body is specified by six unknowns $\boldsymbol{u}=\left(\boldsymbol{u}_{\varphi_{n+1}}, \boldsymbol{\theta}_{n+1}\right) \in U \subset \mathbb{R}^{3} \times \mathbb{R}^{3}$, characterizing the incremental displacement and incremental rotation, respectively. Accordingly, in the present case the 
nodal reparametrization $\boldsymbol{F}_{d}: U \rightarrow C$ introduced in (27) assumes the form

$$
\boldsymbol{q}_{n+1}=\boldsymbol{F}_{d}\left(\boldsymbol{u}_{n+1}, \boldsymbol{q}_{n}\right)=\left[\begin{array}{c}
\boldsymbol{\varphi}_{n}+\boldsymbol{u}_{\varphi_{n+1}} \\
\exp \left(\widehat{\boldsymbol{\theta}_{n+1}}\right) \cdot\left(\boldsymbol{d}_{1}\right)_{n} \\
\exp \left(\widehat{\boldsymbol{\theta}_{n+1}}\right) \cdot\left(\boldsymbol{d}_{2}\right)_{n} \\
\exp \left(\widehat{\boldsymbol{\theta}_{n+1}}\right) \cdot\left(\boldsymbol{d}_{3}\right)_{n}
\end{array}\right],
$$

where Rodrigues' formula is used to obtain a closed form expression of the exponential map, see e.g. [30].

\section{Rigid multibody system dynamics}

The constrained description of rigid bodies in terms of directors is exceptionally well suited for the coupling of several bodies in a multibody system. The body fixed directors offer the possibility to specify the coupling of neighboring bodies by joints constraining their relative motion in a straightforward way. These couplings are termed external constraints. As mentioned in Remark 3.4, using a variational integrator based on the augmented Lagrangian (23) simplifies the elimination of the constraint forces from the system (compared to the use of certain energy-momentum methods), since a discrete null space matrix can directly be inferred from a continuous one by evaluation at $\boldsymbol{q}_{n}$. Therefore, this section summarizes in a concise way the necessary ingredients for the variational integration of the dynamics of kinematic pairs. The idea of this procedure has been presented already in the framework of energy-momentum conserving time integration in [14,15].

Simple kinematic chains as well as tree-structured multibody systems that can be composed by lower kinematic pairs are considered in the sequel. Let a multibody system consist of $N+1$ rigid bodies numbered by $\alpha=0, \ldots, N$ and $N$ axes $\boldsymbol{n}^{0}, \ldots \boldsymbol{n}^{N-1}$, where $\boldsymbol{n}^{\alpha}$ is specified in the $\alpha$-th body frame by

$$
\boldsymbol{n}^{\alpha}=n_{I}^{\alpha} \boldsymbol{d}_{I}^{\alpha} \text {. }
$$

The $N$ joints connecting the bodies are numbered by $\alpha=1, \ldots, N$ and the location of the $\alpha$-th joint in the $(\alpha-1)$-st and $\alpha$-th body is characterized by

$$
\varrho^{\alpha-1, \alpha}=\varrho_{I}^{\alpha-1, \alpha} \boldsymbol{d}_{I}^{\alpha-1}, \quad \varrho^{\alpha, \alpha}=\varrho_{I}^{\alpha, \alpha} \boldsymbol{d}_{I}^{\alpha}
$$

as depicted schematically for two neighboring links in Fig. 8. Note that for tree-structured multibody systems, the two bodies forming a kinematic pair are not necessarily numbered consecutively.

Assuming that none of the links is fixed in space, the multibody system can be described in terms of $n=12(N+1)$ redundant coordinates

$$
\boldsymbol{q}(t)=\left[\begin{array}{c}
\boldsymbol{q}^{0}(t) \\
\vdots \\
\boldsymbol{q}^{N}(t)
\end{array}\right]
$$

generalizing (55). The corresponding constant mass matrix is given by

$$
\boldsymbol{M}=\left[\begin{array}{cccc}
M^{0} & \mathbf{0} & \cdots & \mathbf{0} \\
\mathbf{0} & \boldsymbol{M}^{1} & \cdots & \mathbf{0} \\
\vdots & \vdots & \ddots & \vdots \\
\mathbf{0} & \mathbf{0} & \cdots & M^{N}
\end{array}\right]
$$

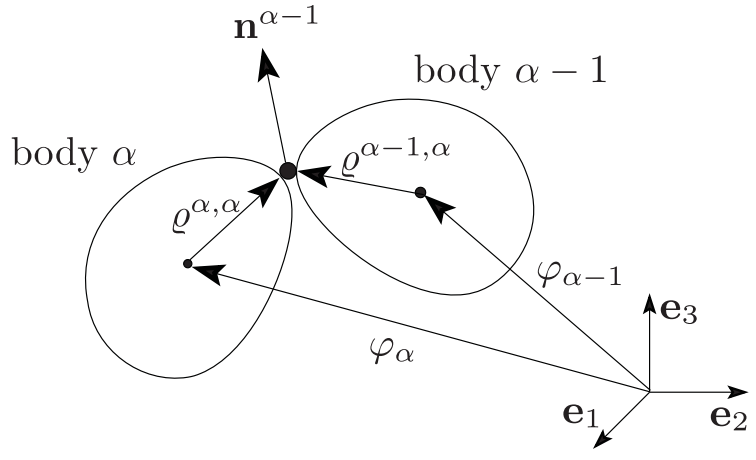

Fig. $8 \alpha$-th pair in a kinematic chain. 
where each submatrix $M^{\alpha} \in \mathbb{R}^{12 \times 12}$ coincides with (58). The rigidity of each link gives rise to six internal constraints $\boldsymbol{g}_{\text {int }}^{\alpha}\left(\boldsymbol{q}^{\alpha}\right) \in \mathbb{R}^{6}$ of the form (56) for $\alpha=0, \ldots, N$. They can be combined to the $m_{\text {int }}=6(N+1)$-dimensional vector of internal constraints $\boldsymbol{g}_{\text {int }}(\boldsymbol{q})$ and the $m_{\text {int }} \times n$ internal constraint Jacobian matrix $\boldsymbol{G}_{\text {int }}(\boldsymbol{q})$.

Similar to the case of a single rigid body treated in Sect. 4 , the twist of a $N+1$ free rigid bodies reads

$$
\boldsymbol{t}=\left[\begin{array}{c}
\boldsymbol{t}^{0} \\
\boldsymbol{t}^{1} \\
\vdots \\
\boldsymbol{t}^{N}
\end{array}\right]
$$

where, analogous to (60), the twist of the $\alpha$-th body $\boldsymbol{t}^{\alpha} \in \mathbb{R}^{6}$, is given by

$$
\boldsymbol{t}^{\alpha}=\left[\begin{array}{c}
\dot{\varphi}^{\alpha} \\
\boldsymbol{\omega}^{\alpha}
\end{array}\right]
$$

Now the redundant velocities $\dot{\boldsymbol{q}} \in \mathbb{R}^{12(N+1)}$ of the multibody system may be expressed as $\dot{\boldsymbol{q}}=\boldsymbol{P}_{\text {int }}(\boldsymbol{q}) \cdot \boldsymbol{t}$, where the $12(N+1) \times 6(N+1)$ matrix $\boldsymbol{P}_{\text {int }}(\boldsymbol{q})$ is given by

$$
\boldsymbol{P}_{\text {int }}(\boldsymbol{q})=\left[\begin{array}{cccc}
\boldsymbol{P}_{\mathrm{int}}^{0}\left(\boldsymbol{q}^{0}\right) & \mathbf{0} & \ldots & \mathbf{0} \\
\mathbf{0} & \boldsymbol{P}_{\mathrm{int}}^{1}\left(\boldsymbol{q}^{1}\right) & \ldots & \mathbf{0} \\
\vdots & \vdots & \ddots & \vdots \\
\mathbf{0} & \mathbf{0} & \cdots & \boldsymbol{P}_{\text {int }}^{N}\left(\boldsymbol{q}^{N}\right)
\end{array}\right]
$$

and $\boldsymbol{P}_{\text {int }}^{\alpha}\left(\boldsymbol{q}^{\alpha}\right)$ is the null space matrix associated with the $\alpha$-th free body, which with regard to (59) reads

$$
\boldsymbol{P}_{\mathrm{int}}^{\alpha}\left(\boldsymbol{q}^{\alpha}\right)=\left[\begin{array}{cc}
\boldsymbol{I} & \mathbf{0} \\
\mathbf{0} & -\widehat{\boldsymbol{d}_{1}^{\alpha}} \\
\mathbf{0} & -\widehat{\boldsymbol{d}_{2}^{\alpha}} \\
\mathbf{0} & -\widehat{\boldsymbol{d}_{3}^{\alpha}}
\end{array}\right] .
$$

Note that by design $\boldsymbol{G}_{\text {int }}(\boldsymbol{q}) \cdot \boldsymbol{P}_{\text {int }}(\boldsymbol{q})=\mathbf{0}$, the $6(N+1) \times 6(N+1)$ zero matrix.

\subsection{Kinematic pairs}

The coupling of two neighboring links in Fig. 8 by a specific joint $J$ yields $m_{\text {ext }}^{(J)}$ external constraints $\boldsymbol{g}_{\text {ext }}^{\alpha}\left(\left[\boldsymbol{q}^{\alpha-1}, \boldsymbol{q}^{\alpha}\right]^{T}\right) \in$ $\mathbb{R}^{m_{\text {ext }}^{(J)}}$. In $[14,15]$, lower kinematic pairs $J \in\{R, P, C, S, E\}$, i.e. revolute, prismatic, cylindrical, spherical and planar pairs have been investigated. Depending on the number of external constraints $m_{\text {ext }}^{(J)}$ they give rise to, the degrees of freedom of the relative motion of one body with respect to the other is decreased from 6 to $r^{(J)}=6-m_{\text {ext }}^{(J)}$. After recalling the derivation of the null space method and nodal reparametrization for kinematic pairs briefly, details are given for the spherical and the revolute pair only, since these are used in the numerical examples presented in Sects. 5.5 and 6.2.

Altogether, $m=m_{\text {int }}+m_{\text {ext }}$ constraints pertaining to the multibody system and the corresponding constraint Jacobians can be combined to

$$
\boldsymbol{g}(\boldsymbol{q})=\left[\begin{array}{l}
\boldsymbol{g}_{\mathrm{int}}(\boldsymbol{q}) \\
\boldsymbol{g}_{\mathrm{ext}}(\boldsymbol{q})
\end{array}\right] \in \mathbb{R}^{m}, \quad \boldsymbol{G}(\boldsymbol{q})=\left[\begin{array}{l}
\boldsymbol{G}_{\mathrm{int}}(\boldsymbol{q}) \\
\boldsymbol{G}_{\mathrm{ext}}(\boldsymbol{q})
\end{array}\right] \in \mathbb{R}^{m \times n} .
$$

The remainder of this section presents details of the external constraints caused by lower kinematic pairs (composed of body 1 and body 2) and their treatment in the framework of the discrete null space method. With the null space matrices for kinematic pairs at hand, a generalization to multibody systems being composed by pairs can be performed easily by respecting formula (6).

Null space matrix. In a kinematic pair, the motion of the second body with respect to an axis fixed in the first body (or with respect to a plane for the planar pair) can be accounted for by introducing $r^{(J)}$ joint velocities $\boldsymbol{\tau}^{(J)}$. Thus the motion of the kinematic pair can be characterized by the independent generalized velocities $\nu^{(J)} \in \mathbb{R}^{6+r^{(J)}}$ with

$$
\boldsymbol{\nu}^{(J)}=\left[\begin{array}{c}
\boldsymbol{t}^{1} \\
\boldsymbol{\tau}^{(J)}
\end{array}\right]
$$


In particular, introducing the $6 \times\left(6+r^{(J)}\right)$ matrix $\boldsymbol{P}_{\mathrm{ext}}^{2,(J)}(\boldsymbol{q})$, the twist of the second body $\boldsymbol{t}^{2} \in \mathbb{R}^{6}$ can be expressed as

$$
\boldsymbol{t}^{2,(J)}=\boldsymbol{P}_{\mathrm{ext}}^{2,(J)}(\boldsymbol{q}) \cdot \boldsymbol{\nu}^{(J)} .
$$

Accordingly, the twist of the kinematic pair can be written in the form

$$
\boldsymbol{t}^{(J)}=\boldsymbol{P}_{\mathrm{ext}}^{(J)}(\boldsymbol{q}) \cdot \boldsymbol{\nu}^{(J)}
$$

with the $12 \times\left(6+r^{(J)}\right)$ matrix $\boldsymbol{P}_{\text {ext }}^{(J)}(\boldsymbol{q})$, which may be partitioned according to

$$
\boldsymbol{P}_{\mathrm{ext}}^{(J)}(\boldsymbol{q})=\left[\begin{array}{cc}
\boldsymbol{I}_{6 \times 6} & \mathbf{0}_{6 \times r^{(J)}} \\
\boldsymbol{P}_{\mathrm{ext}}^{2,(J)}(\boldsymbol{q})
\end{array}\right] .
$$

Once $\boldsymbol{P}_{\text {ext }}^{(J)}(\boldsymbol{q})$ has been established, the total null space matrix pertaining the kinematic pair under consideration can be calculated from

$$
\boldsymbol{P}^{(J)}(\boldsymbol{q})=\boldsymbol{P}_{\mathrm{int}}(\boldsymbol{q}) \cdot \boldsymbol{P}_{\mathrm{ext}}^{(J)}(\boldsymbol{q})=\left[\begin{array}{cc}
\boldsymbol{P}_{\mathrm{int}}^{1}\left(\boldsymbol{q}^{1}\right) & \mathbf{0}_{12 \times r(J)} \\
\boldsymbol{P}_{\mathrm{int}}^{2}\left(\boldsymbol{q}^{2}\right) \cdot \boldsymbol{P}_{\mathrm{ext}}^{2,(J)}(\boldsymbol{q})
\end{array}\right] .
$$

Remark 5.1 (Natural orthogonal complement) Similar to the procedure for the design of appropriate null space matrices outlined above, the relationship between rigid body twists and joint velocities is used in [18] to deduce the "natural orthogonal complement' in the context of simple kinematic chains comprised of elementary kinematic pairs.

Nodal reparametrization. Corresponding to the independent generalized velocities $\boldsymbol{\nu}^{(J)} \in \mathbb{R}^{6+r^{(J)}}$ introduced in (71), the redundant coordinates $\boldsymbol{q} \in \mathbb{R}^{24}$ of each kinematic pair $J \in\{R, P, C, S, E\}$ may be expressed in terms of $6+r^{(J)}$ independent generalized coordinates. Concerning the reparametrization of unknowns in the discrete null space method, relationships of the form

$$
\boldsymbol{q}_{n+1}=\boldsymbol{F}_{d}^{(J)}\left(\boldsymbol{\mu}_{n+1}^{(J)}, \boldsymbol{q}_{n}\right)
$$

are required, where

$$
\boldsymbol{\mu}_{n+1}^{(J)}=\left(\boldsymbol{u}_{\varphi_{n+1}}^{1}, \boldsymbol{\theta}_{n+1}^{1}, \boldsymbol{\vartheta}_{n+1}^{(J)}\right) \in \mathbb{R}^{6+r^{(J)}}
$$

consists of a minimal number of incremental unknowns in $\left[t_{n}, t_{n+1}\right]$ for a specific kinematic pair. In $(77),\left(\boldsymbol{u}_{\varphi_{n+1}}^{1}, \boldsymbol{\theta}_{n+1}^{1}\right) \in$ $\mathbb{R}^{3} \times \mathbb{R}^{3}$ are incremental displacements and rotations, respectively, associated with the first body (see Sect. 4). Furthermore, $\vartheta_{n+1}^{(J)} \in \mathbb{R}^{r^{(J)}}$ denote incremental unknowns which characterize the configuration of the second body relative to the axis (or plane in case of the E pair) of relative motion fixed in the first body. In view of (64), the mapping in (76) may be partitioned according to

$$
\begin{aligned}
& \boldsymbol{q}_{n+1}^{1}=\boldsymbol{F}_{d}^{1}\left(\boldsymbol{u}_{\varphi_{n+1}}^{1}, \boldsymbol{\theta}_{n+1}^{1}, \boldsymbol{q}_{n}^{1}\right), \\
& \boldsymbol{q}_{n+1}^{2}=\boldsymbol{F}_{d}^{2,(J)}\left(\boldsymbol{\mu}_{n+1}^{(J)}, \boldsymbol{q}_{n}\right) .
\end{aligned}
$$

Here, $\boldsymbol{F}_{d}^{1}\left(\boldsymbol{u}_{\varphi_{n+1}}^{1}, \boldsymbol{\theta}_{n+1}^{1}, \boldsymbol{q}_{n}^{1}\right)$ is given by (61). It thus remains to specify the mapping $\boldsymbol{F}_{d}^{2,(J)}\left(\boldsymbol{\mu}_{n+1}^{(J)}, \boldsymbol{q}_{n}\right)$ for each kinematic pair under consideration.

\subsection{Spherical pair}

The $\mathrm{S}$ pair, shown in Fig.9, prevents all relative translation between the two bodies, and thus it gives rise to $m_{\text {ext }}^{(S)}=3$ external constraints of the form

$$
\boldsymbol{g}_{\mathrm{ext}}^{(S)}(\boldsymbol{q})=\varphi^{2}-\varphi^{1}+\varrho^{2}-\varrho^{1}=\mathbf{0}
$$

While the translational motion of the pair can be accounted for by the velocity of one body's center of mass, say by $\dot{\varphi}_{1}$, both bodies can rotate independently. Thus the rotational motion of body 2 is characterized by $r^{(S)}=3$ degrees of freedom. 

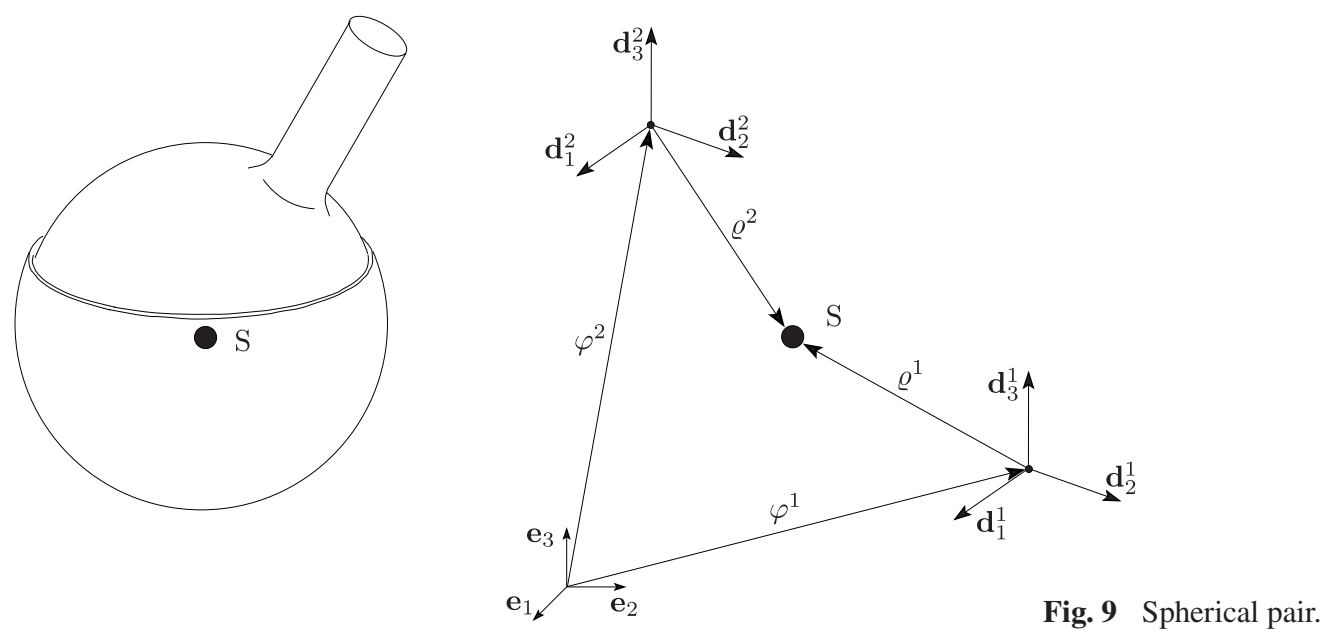

Specifically, with regard to (71) $\tau^{(S)}=\boldsymbol{\omega}^{2}$, the angular velocity of the second body. Accordingly, in the present case, the vector of independent generalized velocities reads

$$
\nu^{(S)}=\left[\begin{array}{c}
\boldsymbol{t}^{1} \\
\boldsymbol{\omega}^{2}
\end{array}\right]
$$

Recall that the twist of the first rigid body given in (67) consists of its translational velocity $\dot{\varphi}^{1}$ and its angular velocity $\boldsymbol{\omega}^{1}$. Taking the time derivative of the external constraints (79) and expressing the redundant velocities in terms of the independent generalized velocities (80) yields

$$
\dot{\varphi^{2}}=\dot{\varphi^{1}}+\omega^{1} \times \varrho^{1}-\omega^{2} \times \varrho^{2} .
$$

Now it can be easily deduced from the relationship $\boldsymbol{t}^{2,(S)}=\boldsymbol{P}_{\mathrm{ext}}^{2,(S)}(\boldsymbol{q}) \cdot \boldsymbol{\nu}^{(S)}$, that

$$
\boldsymbol{P}_{\mathrm{ext}}^{2,(S)}(\boldsymbol{q})=\left[\begin{array}{ccc}
\boldsymbol{I} & -\widehat{\boldsymbol{Q}^{1}} & \widehat{\boldsymbol{Q}^{2}} \\
\mathbf{0} & \mathbf{0} & \boldsymbol{I}
\end{array}\right]
$$

and finally

$$
\boldsymbol{P}_{\mathrm{int}}^{2}\left(\boldsymbol{q}^{2}\right) \cdot \boldsymbol{P}_{\mathrm{ext}}^{2,(S)}(\boldsymbol{q})=\left[\begin{array}{ccc}
\boldsymbol{I} & -\widehat{\varrho^{1}} & \widehat{\boldsymbol{Q}^{2}} \\
\mathbf{0} & \mathbf{0} & -\widehat{\boldsymbol{d}_{1}^{2}} \\
\mathbf{0} & \mathbf{0} & -\widehat{\boldsymbol{d}_{2}^{2}} \\
\mathbf{0} & \mathbf{0} & -\widehat{\boldsymbol{d}_{3}^{2}}
\end{array}\right]
$$

To specify the reduced set of incremental unknowns (77) for the $S$ pair, (80) induces $\boldsymbol{\vartheta}_{n+1}^{(S)}=\boldsymbol{\theta}_{n+1}^{2} \in \mathbb{R}^{3}$, the incremental rotation vector pertaining to the second body. Then the rotational update of the body frame associated with the second body can be performed according to

$$
\left(\boldsymbol{d}_{I}^{2}\right)_{n+1}=\exp \left(\widehat{\boldsymbol{\theta}_{n+1}^{2}}\right) \cdot\left(\boldsymbol{d}_{I}^{2}\right)_{n} .
$$

Enforcing the external constraints (79) at the end of the time-step implies

$$
\varphi_{n+1}^{2}=\varphi_{n+1}^{1}+\varrho_{n+1}^{1}-\varrho_{n+1}^{2} .
$$

Eventually, the last two equations can be used to determine the mapping

$$
\boldsymbol{q}_{n+1}^{2}=\boldsymbol{F}_{d}^{2,(S)}\left(\boldsymbol{\mu}_{n+1}^{(S)}, \boldsymbol{q}_{n}\right)=\left[\begin{array}{cl}
\boldsymbol{\varphi}_{n}^{1}+\boldsymbol{u}_{\varphi_{n+1}}^{1}+ & \exp \left(\widehat{\boldsymbol{\theta}_{n+1}^{1}}\right) \cdot \boldsymbol{\varrho}_{n}^{1}-\exp \left(\widehat{\boldsymbol{\theta}_{n+1}^{2}}\right) \cdot \boldsymbol{\varrho}_{n}^{2} \\
& \exp \left(\widehat{\boldsymbol{\theta}_{n+1}^{2}}\right) \cdot\left(\boldsymbol{d}_{1}^{2}\right)_{n} \\
& \exp \left(\widehat{\boldsymbol{\theta}_{n+1}^{2}}\right) \cdot\left(\boldsymbol{d}_{2}^{2}\right)_{n} \\
& \exp \left(\widehat{\boldsymbol{\theta}_{n+1}^{2}}\right) \cdot\left(\boldsymbol{d}_{3}^{2}\right)_{n}
\end{array}\right] .
$$




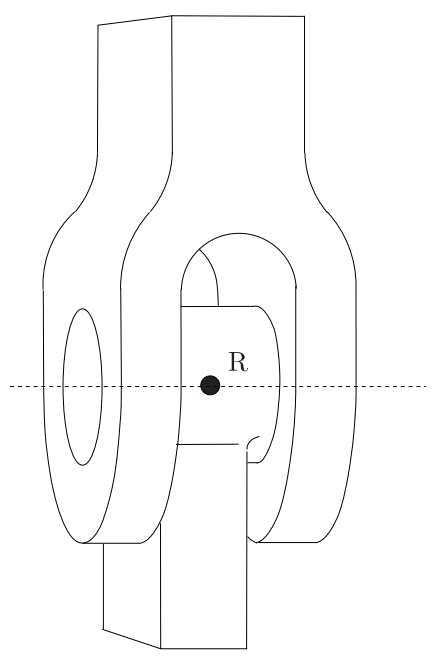

\subsection{Revolute pair}

For the R pair, shown in Fig. 10, a unit vector $\boldsymbol{n}^{1}$ is introduced which is fixed in the first body and specified by constant components $n_{I}^{1}$ with respect to the body frame $\left\{\boldsymbol{d}_{I}^{1}\right\}$

$$
\boldsymbol{n}^{1}=n_{I}^{1} \boldsymbol{d}_{I}^{1}
$$

The R pair entails $m_{\mathrm{ext}}^{(R)}=5$ external constraint functions which may be written in the form

$$
\boldsymbol{g}_{\mathrm{ext}}^{(R)}(\boldsymbol{q})=\left[\begin{array}{c}
\boldsymbol{\varphi}^{2}-\boldsymbol{\varphi}^{1}+\varrho^{2}-\varrho^{1} \\
\left(\boldsymbol{n}^{1}\right)^{T} \cdot \boldsymbol{d}_{1}^{2}-\eta_{1} \\
\left(\boldsymbol{n}^{1}\right)^{T} \cdot \boldsymbol{d}_{2}^{2}-\eta_{2}
\end{array}\right],
$$

where $\eta_{1}, \eta_{2} \in \mathbb{R}$ are constant and need be consistent with the initial conditions.

Remark 5.2 (Singularities in the constrained formulation) It is possible that the constraint Jacobian is singular, when the rotation axis $\boldsymbol{n}^{1}$ is collinear with either of the directors $\boldsymbol{d}_{1}^{2}$ or $\boldsymbol{d}_{2}^{2}$ used to check the fulfillment of the constraints (88). A remedy can be always found in choosing those two directors of $\left\{\boldsymbol{d}_{I}^{2}\right\}$ that are not collinear with $\boldsymbol{n}^{1}$, which is always possible.

The R pair has only one $\left(r^{(R)}=1\right)$ relative degree of freedom which characterizes the rotational motion of the second body relative to the first one. In particular, the kinematic relationship

$$
\omega^{2}=\omega^{1}+\dot{\theta}^{2} n^{1}
$$

holds. The translational velocity of the second body can be expressed as

$$
\dot{\varphi}^{2}=\dot{\varphi}^{1}+\omega^{1} \times\left(\varrho^{1}-\varrho^{2}\right)+\dot{\theta}^{2} \varrho^{2} \times \boldsymbol{n}^{1}
$$

giving rise to

$$
\boldsymbol{P}_{\mathrm{ext}}^{2,(R)}(\boldsymbol{q})=\left[\begin{array}{ccc}
\boldsymbol{I} & \widehat{\varrho^{2}-\varrho^{1}} & \varrho^{2} \times \boldsymbol{n}^{1} \\
\mathbf{0} & \boldsymbol{I} & \boldsymbol{n}^{1}
\end{array}\right]
$$

and

$$
\boldsymbol{P}_{\mathrm{int}}^{2}(\boldsymbol{q}) \cdot \boldsymbol{P}_{\mathrm{ext}}^{2,(R)}(\boldsymbol{q})=\left[\begin{array}{ccc}
\boldsymbol{I} & \widehat{\varrho^{2}-\varrho^{1}} & \varrho^{2} \times \boldsymbol{n}^{1} \\
\mathbf{0} & -\widehat{\boldsymbol{d}_{1}^{2}} & \boldsymbol{n}^{1} \times \boldsymbol{d}_{1}^{2} \\
\mathbf{0} & -\widehat{\boldsymbol{d}_{2}^{2}} & \boldsymbol{n}^{1} \times \boldsymbol{d}_{2}^{2} \\
\mathbf{0} & -\widehat{\boldsymbol{d}_{3}^{2}} & \boldsymbol{n}^{1} \times \boldsymbol{d}_{3}^{2}
\end{array}\right]
$$


The incremental rotational motion of the second body relative to the axis $\boldsymbol{n}^{1}$ is specified by $\vartheta_{n+1}^{(R)}=\theta_{n+1}^{2} \in \mathbb{R}$. The mapping $\boldsymbol{F}_{d}^{2,(R)}\left(\boldsymbol{\mu}_{n+1}^{(R)}, \boldsymbol{q}_{n}\right)$ reads

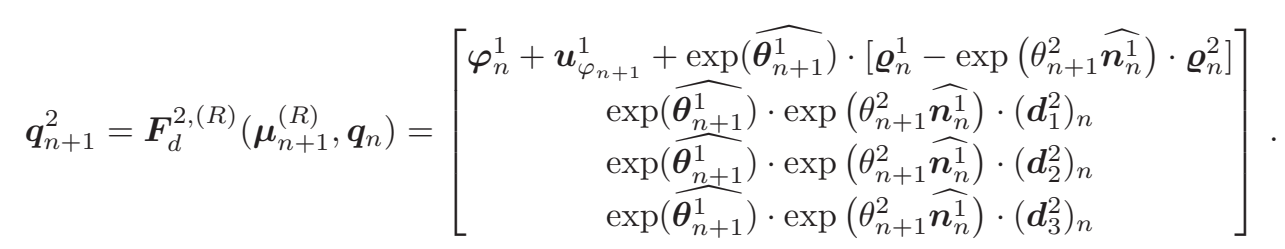

\subsection{Kinematic chains}

Combinations of kinematic pairs constitute kinematic chains. Open kinematic chains can be described as a direct extension of lower kinematic pairs. Thereby the null space matrix pertaining to the coupling joints of the open chain $\boldsymbol{P}_{\mathrm{ext}}^{o}$ consists of blocks that can be inferred from the null space matrices given for the specific joint connections in $[14,15]$. The $n \times\left(n-m^{o}\right)$ null space matrix of the open chain is given by

$$
\boldsymbol{P}^{o}(\boldsymbol{q})=\boldsymbol{P}_{\mathrm{int}}(\boldsymbol{q}) \cdot \boldsymbol{P}_{\mathrm{ext}}^{o}(\boldsymbol{q}),
$$

where $\boldsymbol{P}_{\text {int }}(\boldsymbol{q})$ is given in (68) and $m^{o}=m_{\text {int }}+m_{\text {ext }}^{o}$ is the sum of the number of internal and external constraints.

Unlike for open kinematic chains, where any combination of the lower kinematic pairs can be combined to a movable kinematic chain, the question how many degrees of freedom a closed kinematic chain has, is much more challenging. Generally, it cannot be determined by investigation of the topology of the chain alone (see e.g. [31]). The investigation of closed kinematic chains usually starts with the associated open kinematic chain, which is subject to $m_{\text {ext }}^{c}$ loop closure conditions

$$
\boldsymbol{g}_{\mathrm{ext}}^{c}(\boldsymbol{q})=\mathbf{0}
$$

connecting the first body with the last one in the open chain. It is reasonable to make use of the null space matrix of the open chain by introduction of the muliplicative decomposition

$$
\boldsymbol{P}^{c}(\boldsymbol{q})=\boldsymbol{P}^{o}(\boldsymbol{q}) \cdot \boldsymbol{P}_{\mathrm{ext}}^{c}(\boldsymbol{q}) .
$$

For general closed loop systems, it is often hard or even impossible to find an explicit representation of a continuous null space matrix $\boldsymbol{P}_{\text {ext }}^{c}(\boldsymbol{q})$ by analysis of the independent generalized velocities or in terms of a reparametrization of the constraint manifold. If one succeeds in finding an explicit representation, it can be used in the time-stepping schemes (25), (28). This is in contrast to the treatment of closed kinematic chains in the framework of certain energy-momentum schemes, where highly nonlinear entries in $\boldsymbol{P}_{\text {ext }}^{c}$ make it difficult to infer an explicit representation of a viable discrete null space matrix. In that case one can revert to an implicit representation that has been introduced in [10] and is also described in detail in [15]. According to the procedure described there, an implicit representation of the $\left(n-m^{o}\right) \times\left(n-m^{o}-m_{\text {ext }}^{c}\right)$ discrete null space matrix $\boldsymbol{P}_{\text {ext }}^{c}$ can be found using the QR-decomposition of $\left(\boldsymbol{G}_{\text {ext }}^{c}\left(\boldsymbol{q}_{n}\right) \cdot \boldsymbol{P}^{o}\left(\boldsymbol{q}_{n}\right)\right)^{T}$ (see also Remark 2.2).

Remark 5.3 (Computational costs) The complexity of the QR-decomposition of an $n \times m$ matrix is of the order $n m^{2}$. Since the number of constraints in the open kinematic chain $m^{o}=m_{\text {int }}+m_{\text {ext }}^{o}$ is usually higher than the number of closure conditions $m_{\mathrm{ext}}^{c}$, i.e. $m^{o} \gg m_{\mathrm{ext}}^{c}$, the decomposition of $\mathbb{R}^{n-m^{o}}$ relying on the QR-decomposition of the $\left(n-m^{o}\right) \times m_{\mathrm{ext}}^{c}$ matrix $\left(\boldsymbol{G}_{\text {ext }}^{c}\left(\boldsymbol{q}_{n}\right) \cdot \boldsymbol{P}^{o}\left(\boldsymbol{q}_{n}\right)\right)^{T}$ is substantially cheaper, than the decomposition of $\mathbb{R}^{n}$ based on the QR-decomposition of the $n \times m=n \times\left(m^{o}+m_{\text {ext }}^{c}\right)$ matrix $\boldsymbol{G}^{T}\left(\boldsymbol{q}_{n}\right)$.

\subsection{Six-body linkage}

As an example of a closed loop system, the simple closed kinematic chain consisting of six rigid bodies interconnected by revolute joints in Fig. 11 is considered.

This example has been analyzed kinematically in [32,33]. Simulations of the oscillation of the six-body linkage, whereby the initial configuration as well as the configuration at the turning point lie in one branch of the angle relation in Fig. 12, have been reported by $[34,35]$.

In [36], the reduced equation of motion is deduced symbolically and its sectionwise integration is proposed. Furthermore, the example has been investigated in [14] in the context of an energy-momentum conserving time-stepping scheme. In the sequel, more details than there are given on the coordinate partitioning, which is necessary to work with a reparametrization in one generalized coordinate and leads to an explicit representation of $\boldsymbol{P}_{\mathrm{ext}}^{c}$ for the variational integrator. After that, the performance of the variational scheme using Lagrange multipliers (22) and that using the discrete null space method (28) are compared to the corresponding results from the energy-momentum scheme.

The initial configuration of the six-body linkage forms a cube of side length $l$ (see Fig. 11). 


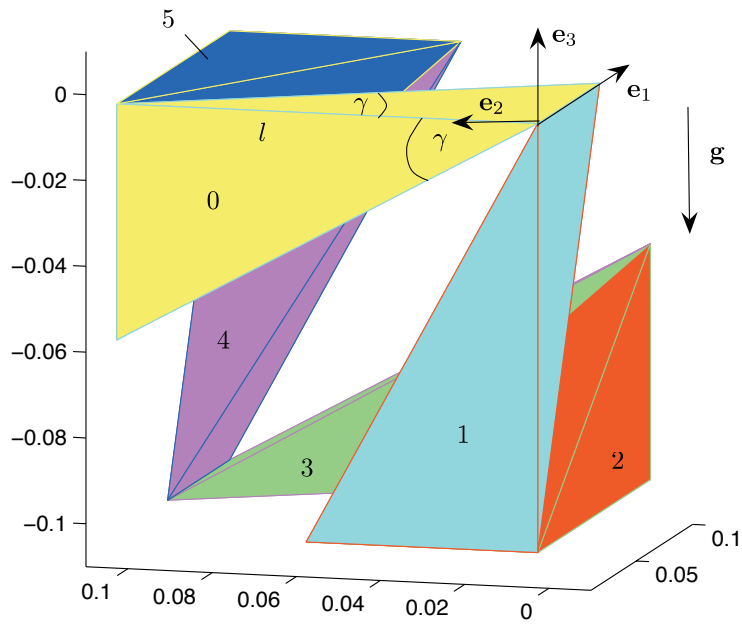

Fig. 11 (online colour at: www.zamm-journal.org) Initial configuration of the six-body linkage.

Basic kinematics and dynamics. Bodies 0,2 , and 4 are identical and bodies 1, 3, and 5 are identical. Furthermore, body 1 is a mirror image of body 0 . Accordingly, it suffices to provide the details of body 0 . Detailed investigation of the multibody's kinematics and symmetries reveals that the linkage moves without collision of contiguous bodies provided that the angle $\gamma$ does not exceed $\pi / 6$ (see [32]). Body 0 has density $\varrho=1000$, length $l=0.1$, and angle $\gamma=0.16 \pi$. Setting $a=\tan \gamma$, in the initial configuration the center of mass of body 0 with respect to the inertial frame $\left\{\boldsymbol{e}_{I}\right\}$ is given by

$$
\varphi^{0}=\varphi_{i}^{0} \boldsymbol{e}_{i}, \quad\left[\varphi_{i}^{0}\right]=\left[\begin{array}{c}
a l / 4 \\
l / 2 \\
-a l / 4
\end{array}\right]
$$

In the inertial frame, the corners of this tetrahedral have the coordinates

$$
\left[c_{i}^{1}\right]=\left[\begin{array}{c}
-a l / 4 \\
-l / 2 \\
a l / 4
\end{array}\right], \quad\left[c_{i}^{2}\right]=\left[\begin{array}{c}
3 a l / 4 \\
-l / 2 \\
a l / 4
\end{array}\right], \quad\left[c_{i}^{3}\right]=\left[\begin{array}{c}
-a l / 4 \\
l / 2 \\
a l / 4
\end{array}\right], \quad\left[c_{i}^{4}\right]=\left[\begin{array}{c}
-a l / 4 \\
l / 2 \\
-3 a l / 4
\end{array}\right],
$$

and the mass of body 0 is given by $M_{\varphi}=\varrho a^{2} l^{3} / 6$. Furthermore, the inertia tensor of body 0 with respect to its center of mass in the initial configuration can be written as

$$
\boldsymbol{J}^{0}=J_{i j}^{0} \boldsymbol{e}_{i} \otimes \boldsymbol{e}_{j}
$$
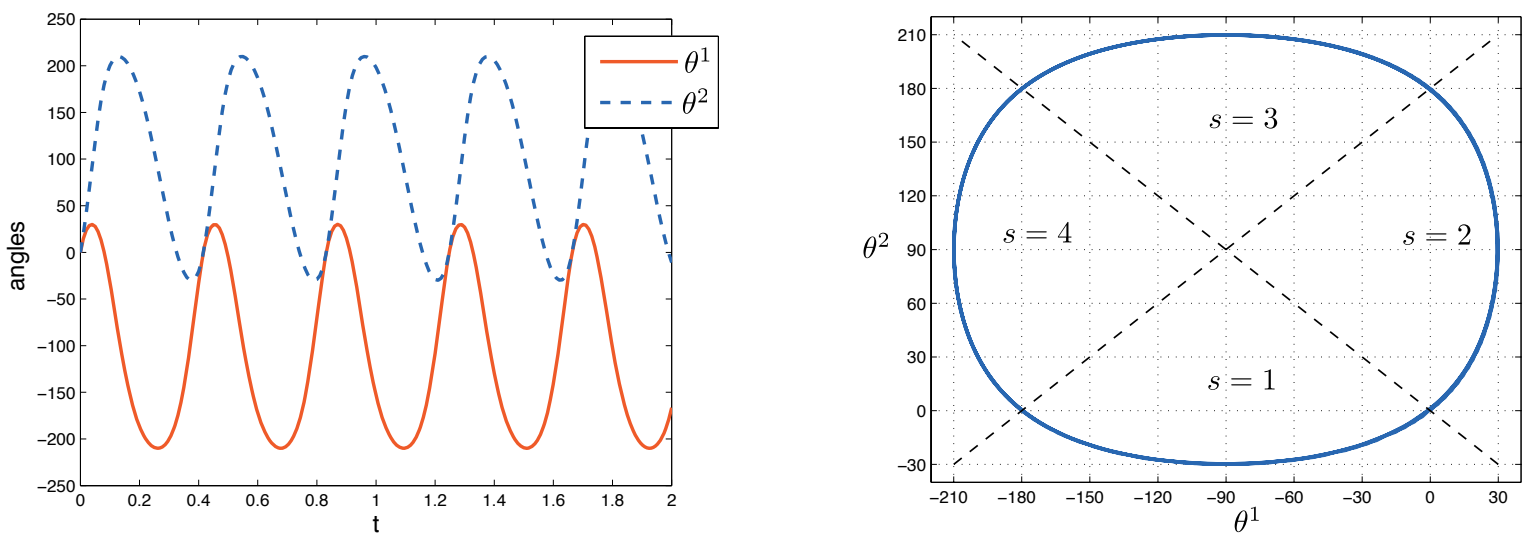

Fig. 12 (online colour at: www.zamm-journal.org) Six-body linkage: relative coordinates $\theta^{1}(t)$ and $\theta^{2}(t)\left(h=10^{-2}\right)$ and angle relation $\theta^{2}\left(\theta^{1}\right)$. 
with

$$
\left[J_{i j}^{0}\right]=\frac{\varrho l^{5} a^{2}}{40}\left[\begin{array}{ccc}
1 / 3+a^{2} / 4 & a / 6 & -a^{2} / 12 \\
a / 6 & a^{2} / 2 & a / 6 \\
-a^{2} / 12 & a / 6 & 1 / 3+a^{2} / 4
\end{array}\right]
$$

The principal values of the inertia tensor with respect to the center of mass are given by

$$
\left[J_{i}^{0}\right]=\frac{a^{2} l^{5}}{120}\left[\left(a^{2}+1\right), \frac{2 a^{2}+1+\sqrt{a^{4}+1}}{2}, \frac{2 a^{2}+1-\sqrt{a^{4}+1}}{2}\right] .
$$

With these preliminaries, the constant symmetric positive definite mass matrix for rigid body 0 can be inferred from (58). For simplicity, the director frame is assumed to coincide with the corresponding principal axes. Their directions relative to the inertial frame are characterized by

$$
\left[\left(\tilde{\boldsymbol{d}}_{1}^{0}\right)_{i}\right]=[-1,0,1], \quad\left[\left(\tilde{\boldsymbol{d}}_{2}^{0}\right)_{i}\right]=\left[1, \frac{a^{2}-1+\sqrt{a^{4}+1}}{a}, 1\right], \quad\left[\left(\tilde{\boldsymbol{d}}_{3}^{0}\right)_{i}\right]=\left[1, \frac{a^{2}-1-\sqrt{a^{4}+1}}{a}, 1\right] .
$$

Then the director frame $\left\{\boldsymbol{d}_{I}^{0}\right\}$ of body 0 is gained by normalization. Together with the placement of the center of mass given in (97), the 12-dimensional configuration variable $\boldsymbol{q}^{0}$ is defined. Subsequent mirroring at the diagonal planes of the cube configuration yields the other components $\boldsymbol{q}^{1}, \ldots, \boldsymbol{q}^{5}$ of the redundant configuration vector $\boldsymbol{q} \in \mathbb{R}^{n}$ of the six body linkage with $n=72$. Let all revolute axes $\boldsymbol{n}^{\alpha}, \alpha=0, \ldots, 5$ be directed inwards the cube and let $\theta^{\alpha}, \alpha=1, \ldots, 6$ be defined as the angle through which body $\alpha$ is rotated relative to body $\alpha-1$ in the positive sense about the axis $\boldsymbol{n}^{\alpha-1}$ of the $\alpha$-th revolute joint. All angles are zero in the cube configuration. Note that body 6 equals body 0 .

In the associated open kinematic chain, the revolute joint connecting body 5 to body 0 is cut, thus six rigid bodies are interconnected by five revolute joints with external constraints $\boldsymbol{g}_{\mathrm{ext}}^{\alpha}\left(\left[\boldsymbol{q}^{\alpha-1}, \boldsymbol{q}^{\alpha}\right]^{T}\right)$ for $\alpha=1, \ldots, 5$ of the form (88). Body 0 is fixed totally in space at its center of mass. The corresponding constraints read

$$
\boldsymbol{g}_{\mathrm{ext}}^{f}(\boldsymbol{q})=\left[\begin{array}{c}
\boldsymbol{\varphi}^{0}-\boldsymbol{c} \\
\boldsymbol{e}_{1}^{T} \cdot \boldsymbol{d}_{1}^{0}-\eta_{1}^{0} \\
\boldsymbol{e}_{2}^{T} \cdot \boldsymbol{d}_{2}^{0}-\eta_{2}^{0} \\
\boldsymbol{e}_{3}^{T} \cdot \boldsymbol{d}_{3}^{0}-\eta_{3}^{0}
\end{array}\right]
$$

where the constant $c \in \mathbb{R}^{3}$ can be inferred from (97). This reduces the independent generalized velocities of the open loop system to the $n-m^{o}=5$ relative angular velocities in the hinges $\nu^{o}=\left[\dot{\theta}^{1}, \ldots, \dot{\theta}^{5}\right]^{T}$ and the independent incremental unknowns of the associated open loop system to $\boldsymbol{\mu}^{o}=\left[\theta^{1}, \ldots, \theta^{5}\right]^{T}$. Note that $\dot{\boldsymbol{q}}^{0}=\mathbf{0} \in \mathbb{R}^{12}$ due to the total fixing in space of body 0 and therefore the first 12 rows in the $72 \times 5$ null space matrix $\boldsymbol{P}^{o}(\boldsymbol{q})$ equal the $12 \times 5$ zero matrix. An explicit representation of the continuous $72 \times 5$ null space matrix of the open chain $\boldsymbol{P}^{o}(\boldsymbol{q})$ could be found by recursive repetition of the kinematic assumptions pertaining to the revolute joint $(89)$ and taking into account $(6)$. See also $[14,15]$ for a representation of the null space matrix of a pure $\mathrm{R}$ chain.

The open loop system is subject to the $m_{\text {ext }}^{c}=5$ additional loop closure constraint $\boldsymbol{g}_{\text {ext }}^{c}(\boldsymbol{q})=\mathbf{0}$, in particular, similar to (88) the closure condition reads

$$
\boldsymbol{g}_{\mathrm{ext}}^{c}(\boldsymbol{q})\left[\begin{array}{c}
\boldsymbol{\varphi}^{5}-\boldsymbol{\varphi}^{0}+\varrho^{5,6}-\varrho^{0,6} \\
\left(\boldsymbol{n}^{5}\right)^{T} \cdot \boldsymbol{d}_{1}^{0}-\eta_{1}^{0} \\
\left(\boldsymbol{n}^{5}\right)^{T} \cdot \boldsymbol{d}_{2}^{0}-\eta_{2}^{0}
\end{array}\right]
$$

The orthonormality conditions (56) on each body's director triad constitute $m_{\text {int }}=36$ internal constraints. Each revolute joint gives rise to $m_{\mathrm{ext}}^{(R)}=5$ external constraints. Together with the fixing in space of body 0 , the number of external constraints is $m_{\mathrm{ext}}=36$, thus the total number of constraints $m=m_{\mathrm{int}}+m_{\mathrm{ext}}=n$ and it is not clear whether the system has any degree of freedom. To answer this question, the dependency of the constraints combined to $\boldsymbol{g}(\boldsymbol{q}) \in \mathbb{R}^{m}$ has to be investigated. To this end it has to be examined whether all configurations in the constraint manifold

$$
\begin{aligned}
C=\left\{\boldsymbol{q} \in \mathbb{R}^{72} \mid \boldsymbol{g}_{\mathrm{int}}^{\alpha}\left(\boldsymbol{q}^{\alpha}\right)=\mathbf{0},\right. & \alpha=0, \ldots, 5, \quad \boldsymbol{g}_{\mathrm{ext}}^{f}(\boldsymbol{q})=\mathbf{0}, \\
\boldsymbol{g}_{\mathrm{ext}}^{\alpha}(\boldsymbol{q})=\mathbf{0}, & \left.\alpha=1, \ldots, 5, \quad \boldsymbol{g}_{\mathrm{ext}}^{c}(\boldsymbol{q})=\mathbf{0}\right\}
\end{aligned}
$$


are regular points, i.e. the rank of the $72 \times 72$ constraint Jacobian $\boldsymbol{G}(\boldsymbol{q})$ has to be calculated for all kinematically admissible configurations $q \in C$. It turns out, that only 71 constraints are independent. Therefore, the number of independent constraints is $\tilde{m}=71$ and the system has $n-\tilde{m}=1$ relative configurational degree of freedom.

Remark 5.4 (Lagrange multiplier method) For the use of the Lagrange multiplier method (22), it is required that the constraint Jacobian has maximal rank. Consequently 71 independent constraints must be used and one constraint equation is omitted. Since the dependence of the constraints changes throughout the motion, the algorithm has to decide dynamically which equation is omitted based on the actual rank of the constraint Jacobian.

The relative configurational degree of freedom can be chosen in $\left\{\theta^{1}, \ldots, \theta^{5}\right\}$. For reasons of symmetry, only two relative angles of the system are nonequal. The following relations hold

$$
\theta^{1}=\theta^{3}=\theta^{5}, \quad \theta^{2}=\theta^{4}=\theta^{6} .
$$

Then the relationship

$$
\sin \left(\theta^{1}\right)\left(1+\sin \left(\theta^{2}\right)\right)=\sin \left(\theta^{2}\right)
$$

can be inferred from the reformulation of the closure constraints (104) in the relative degrees of freedom of the associated open loop system, see [32]. Together with the conditions $\left|\sin \left(\theta^{1}\right)\right| \leq 1$ and $\left|\sin \left(\theta^{2}\right)\right| \leq 1$, (107) yields $\theta^{1} \in\left[-\frac{7 \pi}{6}, \frac{\pi}{6}\right]$ and $\theta^{2} \in\left[-\frac{\pi}{6}, \frac{7 \pi}{6}\right]$.

Fig. 12 shows the relation between $\theta^{1}$ and $\theta^{2}$ for a full revolution of the six-body linkage which bears two problems. First of all, it is ambiguous and secondly, it involves vertical tangents.

Therefore, the reparametrization of the relative angles in one independent relative degree of freedom must be partitioned according to the different periods of the motion as indicated in Fig. 12.

$$
\begin{array}{llll}
s=1 & \theta^{1} \in\left[-\frac{\pi}{2}, 0\right] & \theta^{2}\left(\theta^{1}\right)=\arcsin \left(\frac{\sin \left(\theta^{1}\right)}{1-\sin \left(\theta^{1}\right)}\right) & \in\left[-\frac{\pi}{6}, 0\right], \\
s=2 & \theta^{2} \in\left[0, \frac{\pi}{2}\right] & \theta^{1}\left(\theta^{2}\right)=\arcsin \left(\frac{\sin \left(\theta^{2}\right)}{1+\sin \left(\theta^{2}\right)}\right) & \in\left[0, \frac{\pi}{6}\right], \\
s=3 & \theta^{1} \in\left[-\frac{\pi}{2}, 0\right] & \theta^{2}\left(\theta^{1}\right)=\pi-\arcsin \left(\frac{\sin \left(\theta^{1}\right)}{1-\sin \left(\theta^{1}\right)}\right) & \in\left[\frac{\pi}{2}, \frac{7 \pi}{6}\right], \\
s=4 & \theta^{2} \in\left[0, \frac{\pi}{2}\right] & \theta^{1}\left(\theta^{2}\right)=-\pi-\arcsin \left(\frac{\sin \left(\theta^{2}\right)}{1+\sin \left(\theta^{2}\right)}\right) \in\left[-\frac{7 \pi}{6},-\frac{\pi}{2}\right] .
\end{array}
$$

A consistent reparametrization of all relative rotational degrees of freedom of the associated open kinematic chain $\boldsymbol{\mu}^{o} \in \mathbb{R}^{5}$ reads

$$
\begin{gathered}
s \in\{1,3\} \\
\boldsymbol{\mu}^{o}=\boldsymbol{F}^{s}\left(\theta^{1}\right)=\left[\begin{array}{c}
\theta^{1} \\
\theta^{2}\left(\theta^{1}\right) \\
\theta^{1} \\
\theta^{2}\left(\theta^{1}\right) \\
\theta^{1}
\end{array}\right], \quad \boldsymbol{\mu}^{o}=\boldsymbol{F}^{s}\left(\theta^{2}\right)=\left[\begin{array}{c}
\theta^{1}\left(\theta^{2}\right) \\
\theta^{2} \\
\theta^{1}\left(\theta^{2}\right) \\
\theta^{2} \\
\theta^{1}\left(\theta^{2}\right)
\end{array}\right] .
\end{gathered}
$$

Thus the independent generalized velocities of the closed kinematic chain are given by $\nu^{c}=\dot{\theta}^{1}$ for $s \in\{1,3\}$ and $\nu^{c}=\dot{\theta}^{2}$ for $s \in\{2,4\}$.

The Jacobian of the reparametrization (109) plays the role of a null space matrix. This yields

$$
\begin{array}{rc}
s \in\{1,3\} & s \in\{2,4\} \\
\boldsymbol{\nu}^{o}=\boldsymbol{P}_{\mathrm{ext}}^{c, s} \dot{\theta}^{1} & \boldsymbol{\nu}^{o}=\boldsymbol{P}_{\mathrm{ext}}^{c, s} \dot{\theta}^{2} \\
\boldsymbol{P}_{\mathrm{ext}}^{c, s}=\left[\begin{array}{c}
1 \\
D_{\theta^{1}} \theta^{2} \\
1 \\
D_{\theta^{1}} \theta^{2} \\
1
\end{array}\right], & \boldsymbol{P}_{\mathrm{ext}}^{c, s}=\left[\begin{array}{c}
D_{\theta^{2}} \theta^{1} \\
1 \\
D_{\theta^{2}} \theta^{1} \\
1 \\
D_{\theta^{2}} \theta^{1}
\end{array}\right] .
\end{array}
$$


Using the null space matrix pertaining to the associated open loop system, a continuous null space matrix for the six-body linkage at hand is given by

$$
\boldsymbol{P}^{c}=\boldsymbol{P}^{o}(\boldsymbol{q}) \cdot \boldsymbol{P}_{\mathrm{ext}}^{c}
$$

Numerical results. Consistent initial velocities $\dot{\boldsymbol{q}} \in \mathbb{R}^{72}$ follow from $\dot{\boldsymbol{q}}=\boldsymbol{P}^{c} \dot{\theta}^{1}$ using the continuous null space matrix given in (111). In the numerical example $\dot{\theta}^{1}=30$ has been chosen. Gravity is acting on the system with $g=-9.81$.

Fig. 14 gives an impression of the motion by showing snapshots at consecutive instances.

The evolution of the coordinates $\theta^{1}(t)$ and $\theta^{2}(t)$ is depicted in Fig. 12 as well as the relation of $\theta^{2}\left(\theta^{1}\right)$ described in (108). A characteristic of the motion of the six-body linkage is that the distances $\sigma_{1}, \sigma_{2}, \sigma_{3}$ between opposite corner nodes (the three space diagonals in the initial cube configuration) remain constant (see [37]).

Fig. 13 verifies the algorithmic conservation of these distances. Note that due to the magnitude of the initial angular velocity, the six-body linkage performs full revolutions.

Exact algorithmic conservation of the total energy $H_{d}\left(\boldsymbol{q}_{n}, \boldsymbol{p}_{n}\right)$ using the energy-momentum scheme in conjunction with the discrete null space method in $[14,15]$ is corroborated in Fig. 15.
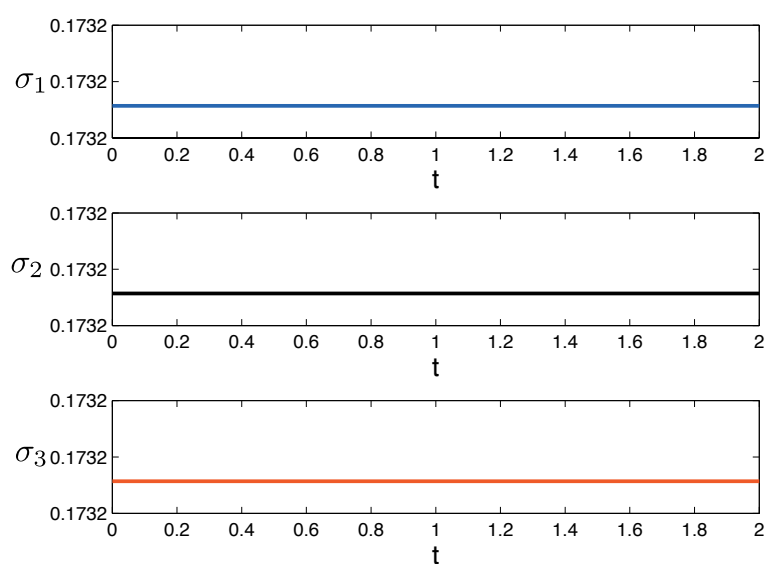

Fig. 13 (online colour at: www.zamm-journal.org) Six-body linkage: distance between opposite corner nodes $\left(h=10^{-2}\right)$.
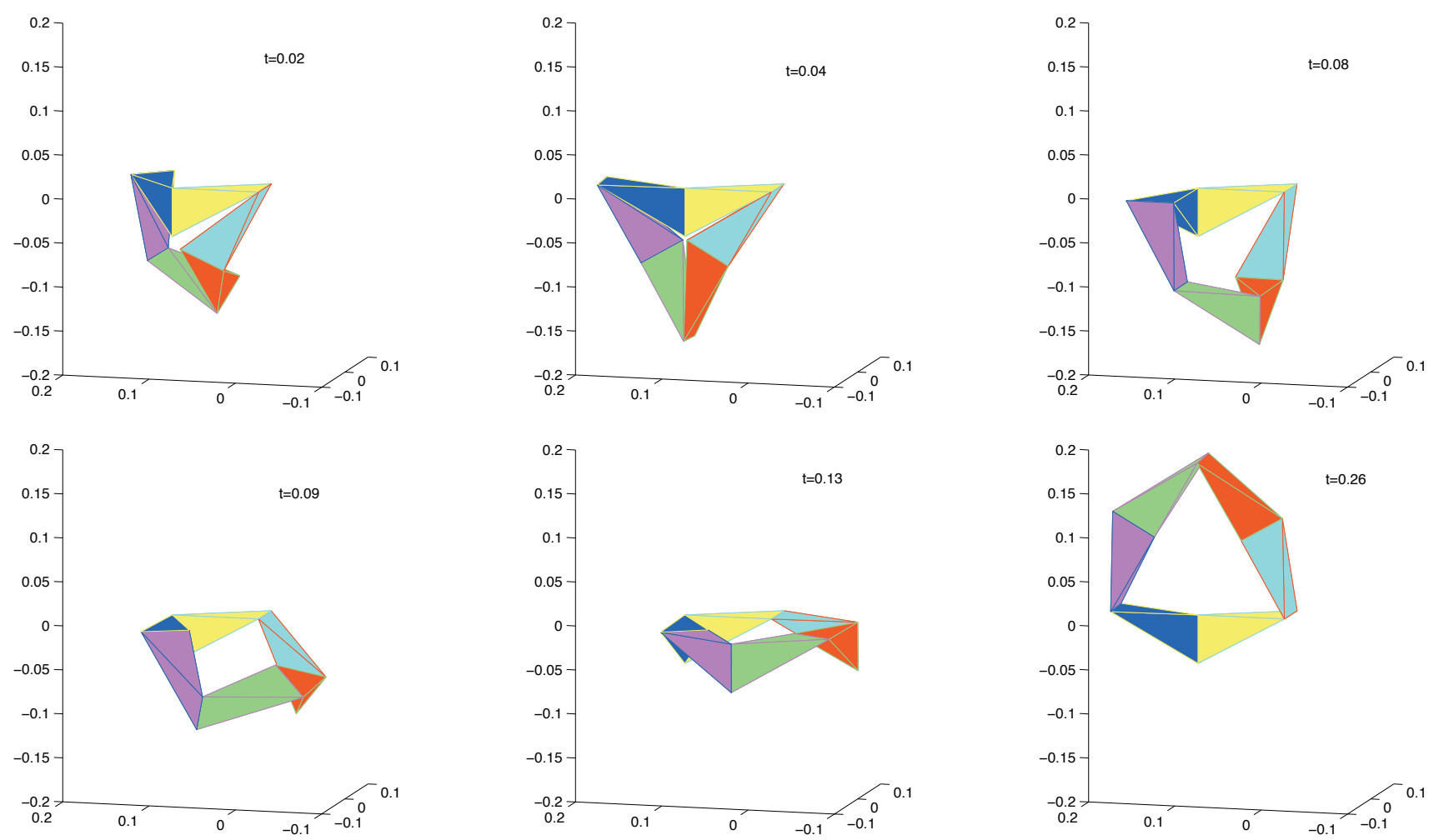

Fig. 14 (online colour at: www.zamm-journal.org) Six-body linkage: snapshots of the motion at $t \in\{0.02,0.04,0.08,0.09,0.13,0.26\}$. 

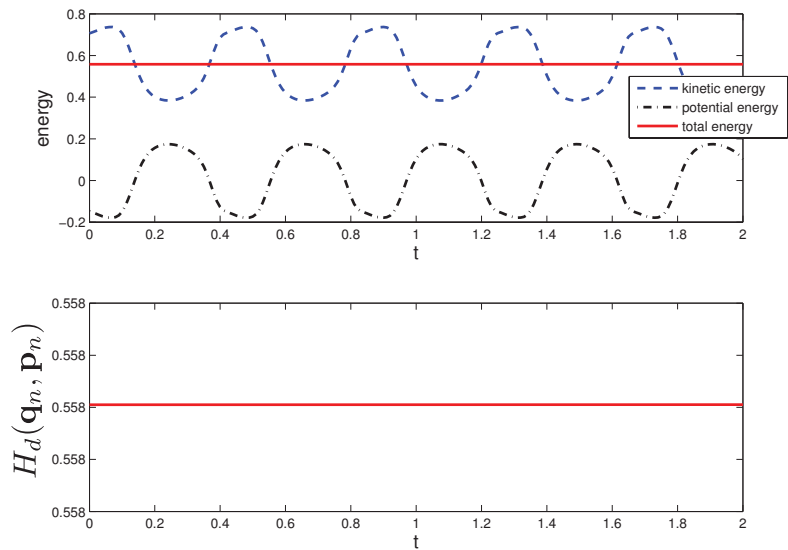

Fig. 15 (online colour at: www.zamm-journal.org) Six-body linkage: energy in terms of $\left(\boldsymbol{q}_{n}, \boldsymbol{p}_{n}\right)$ (energy-momentum scheme, $\left.h=10^{-2}\right)$.
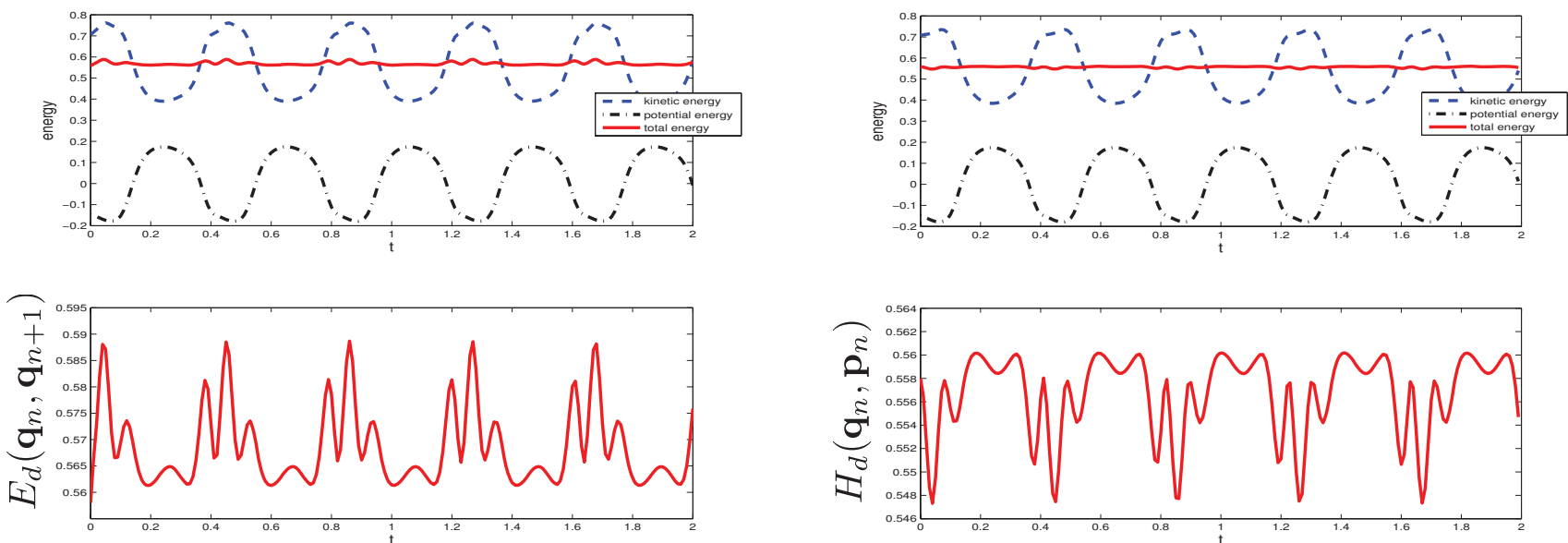

Fig. 16 (online colour at: www.zamm-journal.org) Six-body linkage: energy in terms of $\left(\boldsymbol{q}_{n}, \boldsymbol{q}_{n+1}\right)$ and $\left(\boldsymbol{q}_{n}, \boldsymbol{p}_{n}\right)$ (symplecticmomentum scheme, $h=10^{-2}$ ).
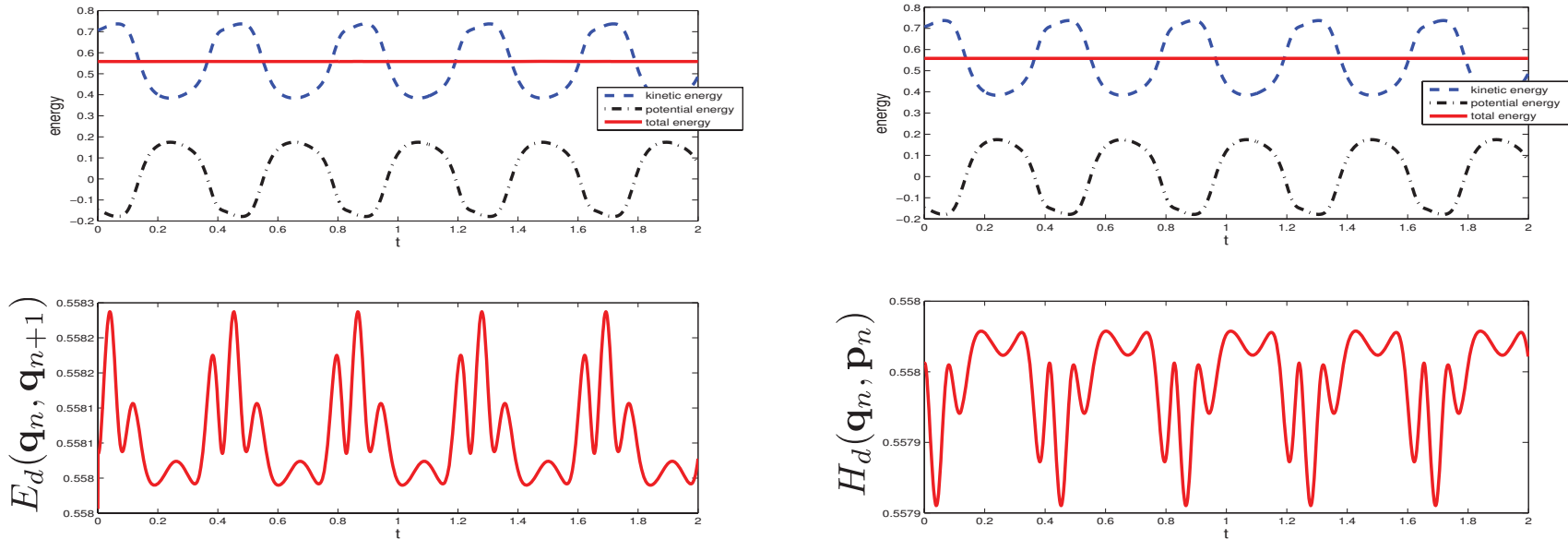

Fig. 17 (online colour at: www.zamm-journal.org) Six-body linkage: energy in terms of $\left(\boldsymbol{q}_{n}, \boldsymbol{q}_{n+1}\right)$ and $\left(\boldsymbol{q}_{n}, \boldsymbol{p}_{n}\right)$ (symplecticmomentum scheme, $h=10^{-3}$ ).

In contrast to that, the evolution of the total energies $E_{d}\left(\boldsymbol{q}_{n}, \boldsymbol{q}_{n+1}\right)$ and $H_{d}\left(\boldsymbol{q}_{n}, \boldsymbol{p}_{n}\right)$ produced by the variational scheme with the discrete null space method in Fig. 16 are not exactly conserved. They oscillate whereby the amplitude of the node based discrete Hamiltonian $H_{d}\left(\boldsymbol{q}_{n}, \boldsymbol{p}_{n}\right)$ is smaller than that of the interval based discrete energy $E_{d}\left(\boldsymbol{q}_{n}, \boldsymbol{q}_{n+1}\right)$.

Both amplitudes get smaller as the time-step decreases, see Fig. 17.

Table 1 shows a comparison of the simulations of the motion of the six-body linkage using the constrained scheme and the d'Alembert-type scheme with nodal reparametrization, respectively for the energy-momentum conserving time-stepping 
Table 1 Six-body linkage: comparison of energy-momentum scheme and symplectic-momentum scheme in combination with Lagrange multipliers and the discrete null space method with nodal reparametrization, respectively.

\begin{tabular}{lcccc}
\hline & \multicolumn{2}{c}{ energy-momentum } & \multicolumn{2}{c}{ symplectic-momentum } \\
& constrained & d'Alembert & constrained & d'Alembert \\
\hline $\begin{array}{l}\text { number of unknowns } \\
n=72, \quad \tilde{m}=71\end{array}$ & 143 & 1 & 143 & 1 \\
\hline CPU-time & 2 & 2 & 1 & 1 \\
\hline condition number & & & & \\
$h=10^{-2}$ & $1 \cdot 10^{5}$ & 1 & $5 \cdot 10^{4}$ & 1 \\
$h=10^{-3}$ & $1 \cdot 10^{8}$ & 1 & $3 \cdot 10^{7}$ & 1 \\
$h=10^{-4}$ & $1 \cdot 10^{11}$ & 1 & $3 \cdot 10^{10}$ & 1 \\
\hline
\end{tabular}

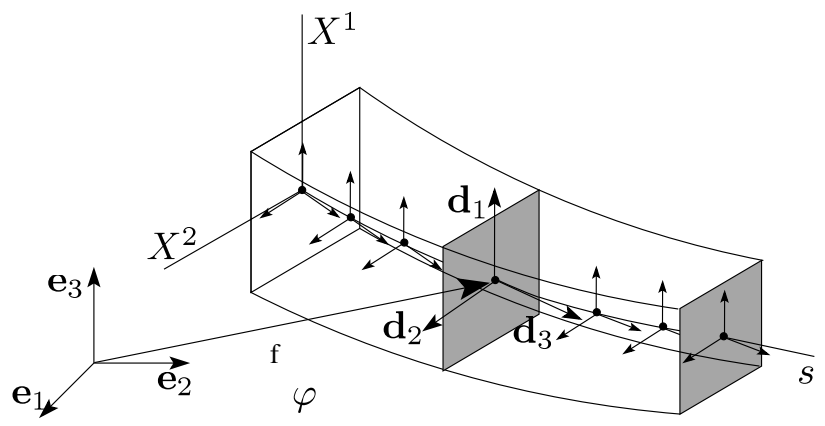

Fig. 18 Configuration of a spatially discretized beam

scheme as well as for the variational integrators (22) and (28). Although a 143-dimensional system of equations has to be solved using the constrained scheme, approximately the same computational time is needed for the setup and solution of the one equation in the d'Alembert-type scheme with nodal reparametrization for both integrators. The reason is that the computation of the null space matrix at each time-step is computational expensive, since it involves the derivative of the angel relation (108). However, the fact that the constraint Jacobian and the null space matrix are evaluated at given configurations $\boldsymbol{q}_{n}$ only in the variational scheme causes the linearization of its nonlinear time-stepping equation to involve less terms, wherefore it is about two times faster than the energy-momentum scheme. Concerning the conditioning issue, the advantageous properties of the advocated discrete null space method are obvious in view of Table 1.

\section{Flexible multibody system dynamics}

In the context of structural mechanics, rigid bodies can be considered as a special case of geometrically exact beams, for which the spatial distribution is degenerated to a single point. In modeling the sequel, the treatment of beam dynamics in the framework of the discrete null space method and its extension to flexible multibody systems presented in [16] will be described briefly.

\subsection{Geometrically exact beam dynamics}

This description of a 'one-node structure' can be extended easily to the modeling of geometrically exact beams as special Cosserat continuum (see [29]). The placement of a material point of the beam reads

$$
\boldsymbol{x}\left(X^{\kappa}, s, t\right)=\boldsymbol{\varphi}(s, t)+X^{\kappa} \boldsymbol{d}_{\kappa}(s, t) .
$$

Here $\left(X^{1}, X^{2}, X^{3}=s\right) \in \mathbb{R}^{3}$ is a triple of curvilinear coordinates with $s \in[0, L] \subset \mathbb{R}$ being the arc-length of the line of centroids $\varphi(s, 0) \in \mathbb{R}^{3}$ in the reference configuration. $\left\{\boldsymbol{d}_{I}\right\}$ represent an orthonormal triad. The directors $\boldsymbol{d}_{\kappa}(s, t), \kappa=1,2$ span a principal basis of the cross-section at $s$ and time $t$ which is accordingly assumed to stay planar. In the reference configuration, $\boldsymbol{d}_{3}(s, 0)$ is tangent to the central line $\varphi(s, 0)$ but this is not necessary in a deformed configuration. This allowance of transverse shear deformation corresponds to the Timoshenko beam theory (see [38]). In contrast to kinematic assumption for the placement of a material point in a rigid body (54), the sum over the repeated index in (112) comprises $\kappa=1,2$ and the spatial extension of the beam in the longitudinal direction is accounted for by the parametrization in $s$. A spatial discretization of the beams configuration (see Fig. 18) in terms of isoparametric finite elements as proposed by [25,39], 
using one-dimensional Lagrange-type nodal shape functions $N_{\alpha}(s)$ reads

$$
\boldsymbol{q}^{h}(s, t)=\sum_{\alpha=1}^{n_{\text {node }}} N_{\alpha}(s) \boldsymbol{q}^{\alpha}(t) \in \mathbb{R}^{12}
$$

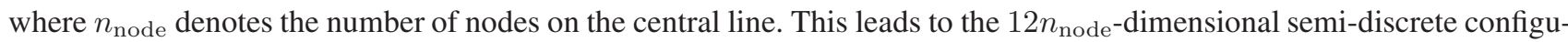
ration vector where the configuration $\boldsymbol{q}^{\alpha}, \alpha=1, \ldots, n_{\text {node }}$ at each node takes the form given in (55). Apparently, a spatially discretized beam can be interpreted as a chain of rigid bodies for which the interconnections are prescribed by the connectivity of the spatial finite element method, see e.g. [40]. In the sequel, a rigid body is considered as a special semi-discrete beam, consisting of only one node, i.e. $n_{\text {node }}=1$. The internal (orthonormality) constraints $\boldsymbol{g}_{\text {int }}: \mathbb{R}^{12 n_{\text {node }}} \rightarrow \mathbb{R}^{m_{\text {int }}}$ with $m_{\text {int }}=6 n_{\text {node }}$, pertaining to the underlying continuous theory are of the form (56) for $\alpha=1, \ldots, n_{\text {node }}$.

An inherent property of the interpolation (113) is that the constraints on the director triads are relaxed to the nodes of the mesh.

Remark 6.1 Many current semi-discrete beam formulations avoid the introduction of internal constraints by using rotational degrees of freedom, see e.g. [41,42]. However it has been shown by Chrisfield and Jelenic [43] that the interpolation of non-commutative finite rotations bears the risk of destroying the objectivity of the strain measures in the semi-discrete model. This can be circumvented by the spatial interpolation of the director triad in (113) as proposed independently in [39] and [25].

The redundant velocities $\dot{\boldsymbol{q}} \in \mathbb{R}^{12 n_{\text {node }}}$ of the semi-discrete beam may be expressed in terms of the $6 n_{\text {node-dimensional }}$ twist as $\dot{\boldsymbol{q}}=\boldsymbol{P}_{\text {int }}(\boldsymbol{q}) \cdot \boldsymbol{t}$, where the $12 n_{\text {node }} \times 6 n_{\text {node }}$ internal null space matrix $\boldsymbol{P}_{\text {int }}(\boldsymbol{q})$ has the same block structure as (68) with nodal internal null space matrices (69) and the nodal reparametrization $\boldsymbol{q}_{n+1}^{\alpha}=\boldsymbol{F}_{d}\left(\boldsymbol{u}_{n+1}^{\alpha}, \boldsymbol{q}_{n}^{\alpha}\right)$ assumes the form (61).

Differentiating the placement of a material point (112) in time, one realizes the fact that the beam's kinetic energy is independent of $\dot{\boldsymbol{d}}_{3}$. Due to that property, the Lagrangian is degenerate and it follows that $\boldsymbol{p}_{3}=\partial L / \partial \dot{\boldsymbol{d}}_{3}=\mathbf{0}$. The kinetic energy is computed in terms of the 9-dimensional reduced velocity $\dot{\overline{\boldsymbol{q}}}=\left[\dot{\boldsymbol{\varphi}}, \dot{\boldsymbol{d}}_{1}, \dot{\boldsymbol{d}}_{2}\right]^{T}$ or the reduced conjugate momentum vector $\overline{\boldsymbol{p}}=\left[\boldsymbol{p}_{\varphi}, \boldsymbol{p}_{1}, \boldsymbol{p}_{2}\right]^{T}$ and the non-singular reduced $9 \times 9$ mass matrix

$$
\overline{\boldsymbol{M}}=\left[\begin{array}{ccc}
A_{\rho} \boldsymbol{I} & \mathbf{0} & \mathbf{0} \\
\mathbf{0} & M_{\rho}^{1} \boldsymbol{I} & \mathbf{0} \\
\mathbf{0} & \mathbf{0} & M_{\rho}^{2} \boldsymbol{I}
\end{array}\right],
$$

where $\boldsymbol{I}$ and $\mathbf{0}$ denote the $3 \times 3$ identity and zero matrices respectively, $A_{\rho}$ is the mass density per reference length and $M_{\rho}^{1}, M_{\rho}^{2}$ can be interpreted as principal mass-moments of inertia of the cross-section. See e.g. [25,44-46] for the transition to the mass matrix of the spatially discrete beam formulation.

In a temporally discrete Lagrangian $L_{d}\left(\boldsymbol{q}_{n}, \boldsymbol{q}_{n+1}\right)$ that is formulated in terms of the $12 n_{\text {node-dimensional configuration }}$ vector, a $12 n_{\text {node }} \times 12 n_{\text {node }}$ singular mass matrix has to be used whose entries corresponding to $\boldsymbol{d}_{3}^{\alpha}$ are zero. Note that this does not lead to problems in determining $\boldsymbol{d}_{3}^{\alpha}$ from the equations of motion since of course the stored deformation energy function and the resulting internal forces do depend on $\boldsymbol{d}_{3}^{\alpha}$. Therefore one has to be careful in computing only $\overline{\boldsymbol{p}}_{n}^{\alpha}$ using the discrete Legendre transforms (34), (35). The hidden constraints on momentum level (46) now read

$$
\overline{\boldsymbol{h}}_{d}\left(\boldsymbol{q}_{n}, \overline{\boldsymbol{p}}_{n}\right)=\overline{\boldsymbol{G}}\left(\boldsymbol{q}_{n}\right) \cdot \overline{\boldsymbol{M}}^{-1} \cdot \overline{\boldsymbol{p}}_{n}=\mathbf{0},
$$

where in $\overline{\boldsymbol{G}}\left(\boldsymbol{q}_{n}\right)$ consists of those rows and columns in $\boldsymbol{G}\left(\boldsymbol{q}_{n}\right)$ that are not related to $\boldsymbol{d}_{3}^{\alpha}$. Accordingly, in the projected discrete Legendre transforms (39), (40), the projection reads

$$
\overline{\boldsymbol{Q}}=\boldsymbol{I}_{9 n_{\text {node }} \times 9 n_{\text {node }}}-\overline{\boldsymbol{G}}^{T} \cdot\left[\overline{\boldsymbol{G}} \cdot \overline{\boldsymbol{M}}^{-1} \cdot \overline{\boldsymbol{G}}^{T}\right]^{-1} \overline{\boldsymbol{G}} \cdot \overline{\boldsymbol{M}}^{-1} .
$$

\subsection{Flexible multibody systems: the three-bar swing}

The description of rigid bodies and spatially discretized geometrically exact beams as constrained continua in terms of the configuration variables given in (55) allows their coupling to a multibody system consisting of rigid and elastic components in a systematic way as described in [16]. Similar to (64), the configuration vectors of all components in the multibody system are combined into the general configuration vector $\boldsymbol{q}(t) \in \mathbb{R}^{n}$ where $n$ is a 12 times the actual number of spatial nodes present in the semi-discrete system. The external constraints can arise e.g. due to the coupling of neighboring components by joints, a rigid connection representing the intersection of beams or standard Dirichlet boundary conditions.

As a specific example, the three-bar swing shown in Fig. 19 is considered. It consists of an elastic beam hinged at its 


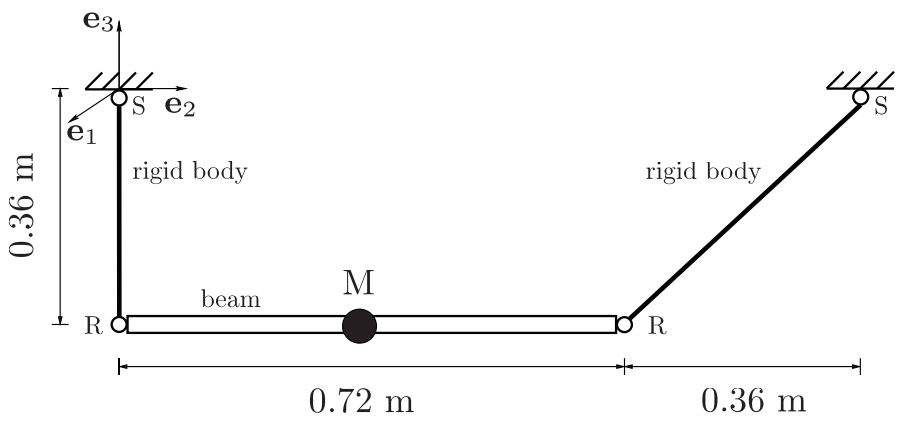

Fig. 19 Three-bar swing comprising a flexible beam with midspan mass hinged (by revolute joints R) to rigid bodies fixed in space (by spherical joints $\mathrm{S}$ ).

ends to rigid bodies by revolute joints. The rigid bodies are fixed in space by spherical joints. An additional point mass is concentrated at the beams mid-point.

This example has been investigated previously in [47] using an energy-conserving scheme and the generalized- $\alpha$ method. In [48] results from an energy-conserving and an energy-decaying scheme are presented. Here, motion and deformation are predicted by the variational integrator and compared to simulations using the energy-momentum scheme based on the discrete derivative [20]. Both integrators have been implemented using Lagrange multipliers as well as the discrete null space method to treat the constraints. See [16] for a detailed introduction to the energy-momentum conserving integration of flexible multibody dynamics where constraints are treated by the discrete null space method. Besides geometrically exact beams, also shells are treated in [16].

A major difference in the performances of the symplectic-momentum conserving variational integrator and the energymomentum scheme is the capability the simulate stiff problems, i.e. ODEs possessing a wide spectrum of frequencies. While the energy-momentum scheme can reproduce the results in $[47,48]$ for the set of material parameters used there $\left(E=73 \cdot 10^{9} \mathrm{~N} / \mathrm{mm}^{2}, \nu=0.3, \rho=2700 \mathrm{~kg} / \mathrm{mm}^{3}\right)$ for a beam of rectangular cross-section $(5 \mathrm{~mm} \times 1 \mathrm{~mm})$ oriented such that the smaller of the two bending stiffnesses is about the major bending axis, no time-step between $10^{-1} \mathrm{~s}$ and $10^{-5} \mathrm{~s}$ could be found for which the symplectic-momentum scheme was able to simulate a period of motion that includes the 'event X', as it is called in [47] in which the rigid link on the right reverses its direction of rotation, causing highly vibratory behavior of the beam. All attempts resulted in blow up of the total energy at the 'event X'. This indicates in the same direction as the arguments in [49] saying that temporally unresolved high frequencies are 'seen by the [symplectic] algorithm as infinite sample frequencies leading inevitably to [...] instabilities. In sharp contrast with this result, [...] the energy-momentum conserving scheme remains stable'. However, it is not guaranteed that the energy-momentum scheme distributes the energy over the frequency spectrum correctly. One possibility to deal with a large spectrum of frequencies in a problem is to use asynchronous variational integration [50]. The idea is to assign smaller time-steps to small elements in the region where high frequency motion is expected. In order not to slow down the overall simulation unnecessarily, larger elements in regions performing slower deformation are integrated using a larger time-step. In [51], this method enables the long term integration of the motion of a long thin helicopter blade for different sets of material parameters ranging from very stiff almost rigid motion to rather soft material behavior. Another approach to address the capturing of high frequency motion in the overall motion is to approximate the high order modes (e.g. by Fourier transform of the motion of nodes on a fine subgrid as it is done in [52]) such that one can explicitly calculate a time-dependent force that represents the influence of the higher order modes on the main mode.

The simulations described in the sequel use material properties of a less stiff material with higher density. Both rigid bodies' mass is $0.01 \mathrm{~kg}$ and they have the shape of pyramids with a square ground face of edge length $0.02 \mathrm{~m}$ and the height of $0.36 \mathrm{~m}$ and $0.36 \sqrt{2} \mathrm{~m}$, respectively.

A concentrated mass of $M=5 \mathrm{~kg}$ is rigidly connected at the midspan node of the beam, which is discretized by 20 linear finite beam elements. The semi-discrete beam's response to loading is based on hyperelastic material behavior with stiffness parameters $G A=175480.7692 \mathrm{~N}, E A=547500 \mathrm{~N}, E I_{1}=114.0625 \mathrm{Nm}^{2}, E I_{2}=10.2656 \mathrm{Nm}^{2}$, and $G J=13.7401 \mathrm{Nm}^{2}$. The sectional mass properties are $A_{\rho}=7500 \mathrm{~kg} / \mathrm{m}, M_{\rho}^{1}=1.5625 \mathrm{~kg} \mathrm{~m}$, and $M_{\rho}^{2}=0.1406 \mathrm{~kg} \mathrm{~m}$. The cross section is oriented such that the smaller of the two bending stiffnesses is with respect to the axis parallel to $e_{1}$. (Note that the numbering of the bending stiffnesses corresponds to the numbering of the nodal director triads which differ from the inertial frame.) The loading is a triangular pulse in $e_{2}$-direction which is applied at the midspan mass. It starts with $0 \mathrm{~N}$ at $t=0 \mathrm{~s}$, peaks with $10000 \mathrm{~N}$ at $t=0.125 \mathrm{~s}$ and ends with $0 \mathrm{~N}$ at $t=0.25 \mathrm{~s}$.

Snapshots of the motion and deformation are depicted in Fig. 21. The elements' colors represent a linear interpolation in space of the weighted sum of the resulting axial and shear forces norm and the resulting bending and torsional moments norm. Thereby blue represents zero and red represents 4000. According to the loading in axial direction of the beam, the axial forces dominate the stress resultants therefore the resulting moments have been scaled by a factor of 100 . 

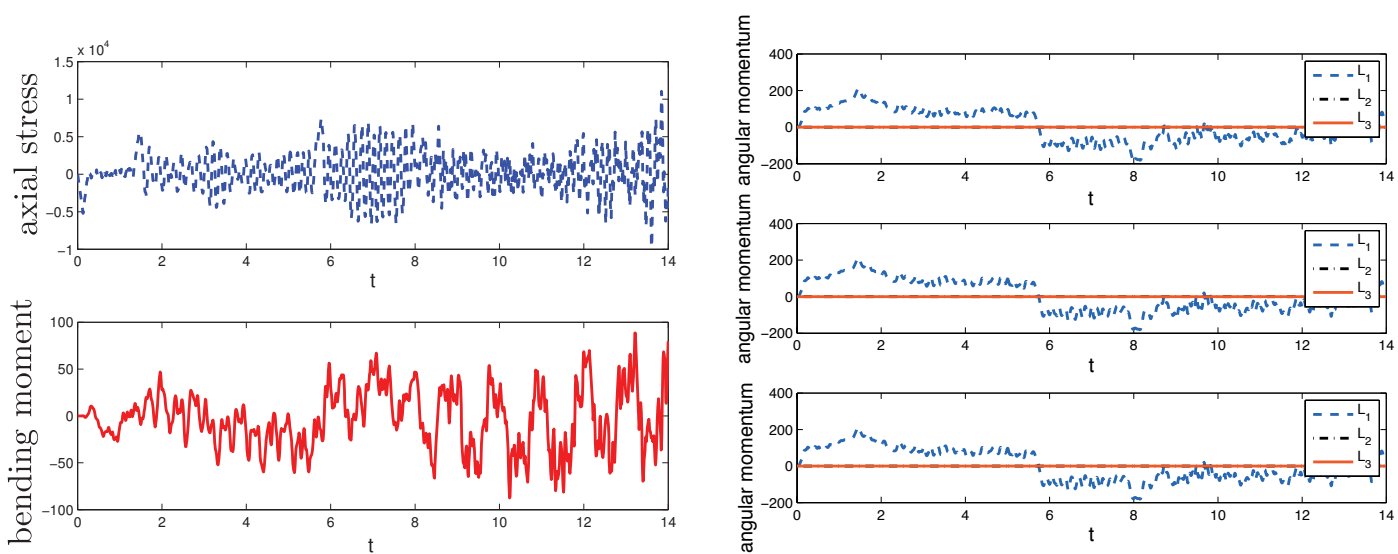

Fig. 20 (online colour at: www.zamm-journal.org) Three-bar swing: axial force and bending moment with respect to $\mathbf{e}_{1}$ in the element to the right of the concentrated mass (left) and components of angular momentum $\boldsymbol{L}=L_{I} \boldsymbol{e}_{I}$ in terms of $\left(\boldsymbol{q}_{n}, \boldsymbol{q}_{n+1}\right),\left(\boldsymbol{q}_{n}, \boldsymbol{p}_{n}\right)$ and $\left(\boldsymbol{q}_{n},{ }^{Q} \boldsymbol{p}_{n}\right)$ (right, top to bottom), (symplectic-momentum scheme, $h=10^{-2}$ ).
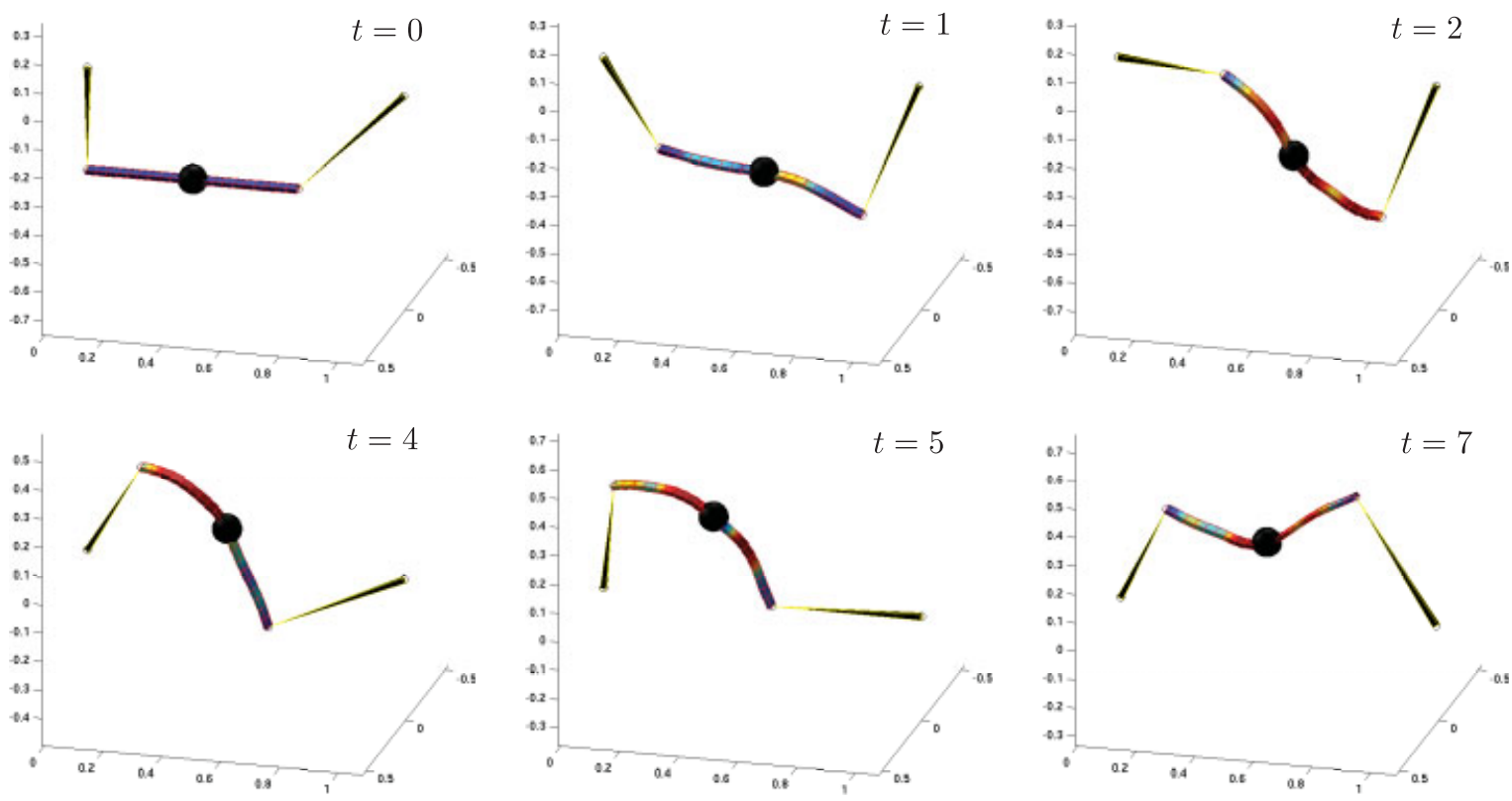

Fig. 21 (online colour at: www.zamm-journal.org) Three-bar swing: snapshots of the motion and deformation (symplecticmomentum scheme, $h=10^{-2}$ ).

The evolution of the axial force and bending moments with respect to the axis $e_{1}$ in the element to the right of the concentrated mass can be observed from the left diagram in Fig. 20.

This figure shows the high frequency oscillations after the 'event $\mathrm{X}$ ' at $t \approx 1.4 \mathrm{~s}$. The diagram on the right hand side illustrates that the motion really takes place in the $\left(\boldsymbol{e}_{2}, \boldsymbol{e}_{3}\right)$-plane (even though a fully three-dimensional model is used) since the only non-zeros component of the angular momentum is that with respect to the out of plane axis $e_{1}$. The left hand diagram in Fig. 22 reveals again the good energy behavior of the variational scheme.

The diagram on the right hand side of this figure illustrates the orbit of the concentrated mass in the $\left(\boldsymbol{e}_{2}, \boldsymbol{e}_{3}\right)$-plane. One can see clearly how the beams deformation superposes the overall rigid motion of the multibody system. Fig. 23 shows that after the vanishing of the external load, energy is conserved exactly by the energy-momentum scheme.

On the other hand, as expected, the symplectic-momentum scheme shows oscillations with decreasing amplitude for decreasing time-steps, see Fig. 24.

The results presented here have been obtained using the discrete null space method, i.e. (25) with reparametrization has been solved. The Lagrange multiplier method (22) has been implemented as well. Since both schemes are equivalent, the resulting diagrams are indistinguishable. However, the constrained scheme (22) behaves differently with regard to 

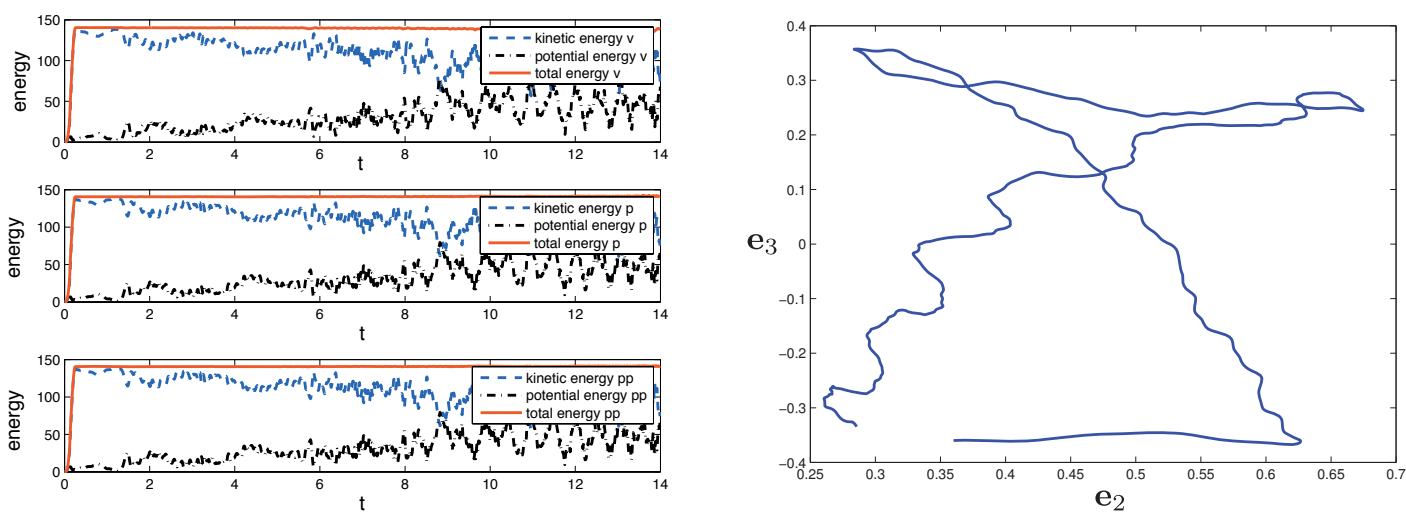

Fig. 22 (online colour at: www.zamm-journal.org) Three-bar swing: energy in terms of $\left(\boldsymbol{q}_{n}, \boldsymbol{q}_{n+1}\right),\left(\boldsymbol{q}_{n}, \boldsymbol{p}_{n}\right)$ and $\left(\boldsymbol{q}_{n},{ }^{Q} \boldsymbol{p}_{n}\right)$ (top to bottom) and orbit of the concentrated mass in the $\left(\boldsymbol{e}_{2}, \boldsymbol{e}_{3}\right)$-plane (symplectic-momentum scheme, $\left.h=10^{-2}\right)$.
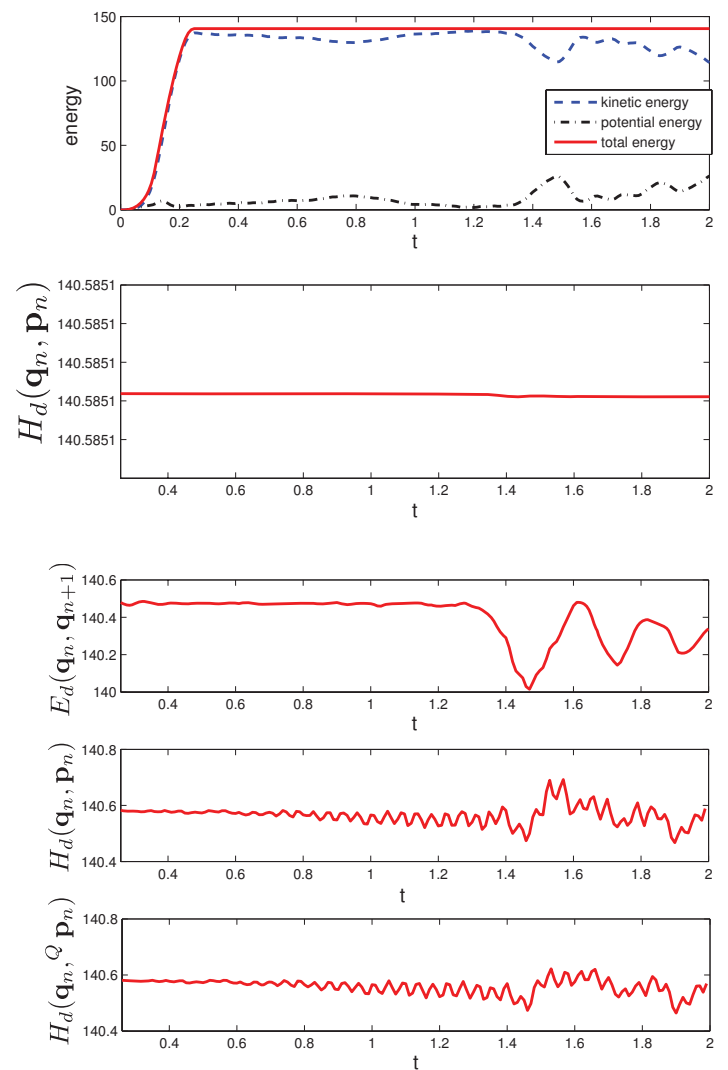

Fig. 23 (online colour at: www.zamm-journal.org) Three-bar swing: energy in terms of $\left(\boldsymbol{q}_{n}, \boldsymbol{p}_{n}\right)$ (energy-momentum scheme, $h=10^{-2}$ ).
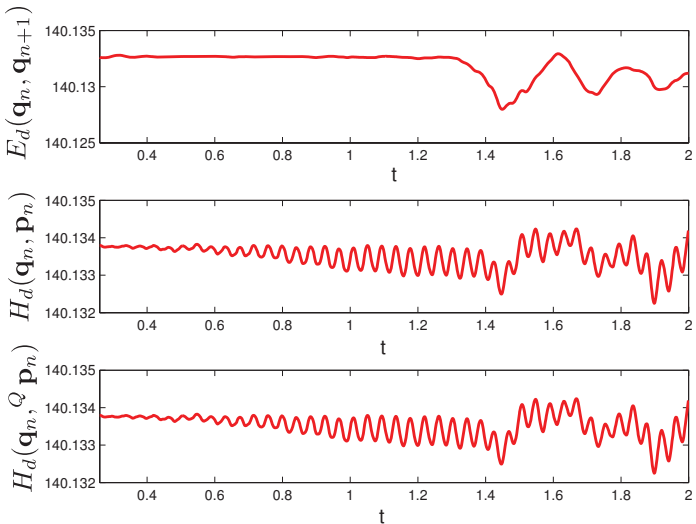

Fig. 24 (online colour at: www.zamm-journal.org) Three-bar swing: total energy for $h=10^{-2}$ (left) and $h=10^{-3}$ (right), (symplectic-momentum scheme).

computational costs and the condition number of the iteration matrix, see Table 2. The same problem has also been simulated using the energy-momentum scheme in conjunction with the Lagrange multiplier method and the discrete null space method with nodal reparametrization. Table 2 also compares the performance of these simulations to that of the d'Alembert-type symplectic-momentum scheme with reparametrization based on performing 1000 steps with different time-steps. It reveals that the solution of the larger dimensional constrained system takes longer than that of the d'Alembert-type scheme with local reparametrization with a relatively simple null space matrix. One can also observe that the more complicated linearization of the energy-momentum scheme requires more computational time. Furthermore, condition numbers increase strongly for decreasing time-steps in both Lagrange multiplier schemes while they remain of moderate value in the d'Alemberttype schemes. 
Table 2 Three-bar swing: comparison of energy-momentum scheme and symplectic-momentum scheme in combination with Lagrange multipliers and the discrete null space method with nodal reparametrization, respectively.

\begin{tabular}{lcccc}
\hline & \multicolumn{2}{c}{ energy-momentum } & \multicolumn{2}{c}{ symplectic-momentum } \\
& constrained & d'Alembert & constrained & d'Alembert \\
\hline $\begin{array}{l}\text { number of unknowns } \\
n=276, \quad m=154\end{array}$ & 430 & 122 & 430 & 122 \\
\hline CPU-time & 1.5 & 1.2 & 1.4 & 1 \\
\hline $\begin{array}{l}\text { condition number } \\
h=10^{-2}\end{array}$ & $2 \cdot 10^{12}$ & $4 \cdot 10^{4}$ & $6 \cdot 10^{11}$ & $5 \cdot 10^{4}$ \\
$h=10^{-3}$ & $2 \cdot 10^{14}$ & $9 \cdot 10^{4}$ & $5 \cdot 10^{13}$ & $9 \cdot 10^{4}$ \\
$h=10^{-4}$ & $2 \cdot 10^{17}$ & $2 \cdot 10^{5}$ & $5 \cdot 10^{16}$ & $2 \cdot 10^{5}$ \\
\hline
\end{tabular}
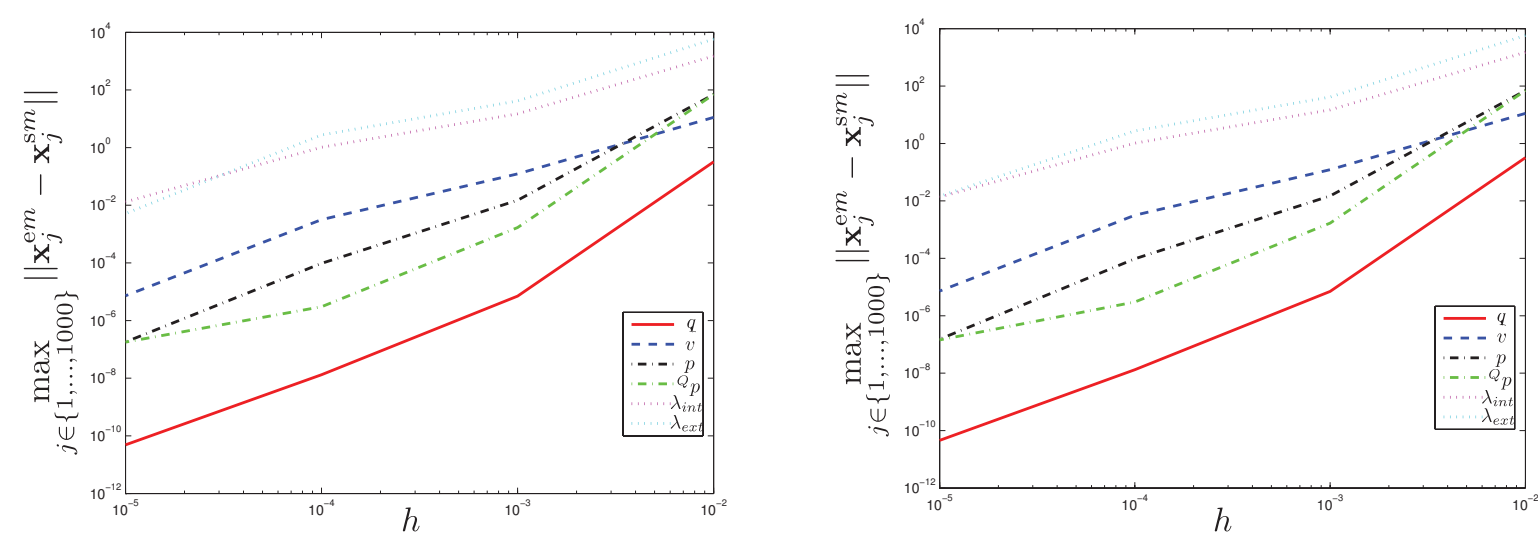

Fig. 25 (online colour at: www.zamm-journal.org) Three-bar swing: convergence of trajectories computed by the energymomentum scheme with Lagrange multipliers (left) and with the discrete null space method with nodal reparametrization (right) to trajectories obtained by the symplectic-momentum scheme and vice versa.

Besides the analytical proof of convergence of variational integrators for constrained problems, in [23] the same example is used to verify the second order convergence numerically. A convergence study is not repeated here, however the final Fig. 25 shows that based on performing 1000 steps with decreasing time-steps, the trajectories of the configuration, velocity, conjugate momentum, projected conjugate momentum and Lagrange multipliers corresponding to the internal and external constraints, obtained by the energy-momentum conserving scheme using either Lagrange multipliers or the discrete null space method with nodal reparametrization, do approach the trajectories predicted by the variational scheme and vice versa.

\section{Conclusion}

The definition of the augmented discrete Lagrangian (23) is crucial for the variational integration of constrained systems. It influences the form of the discrete Legendre transform, which is necessary for the consistent initialization of the simulation as well as for postprocessing steps when evaluating the discrete trajectory.

The discrete null space method, which was originally used with an energy-momentum conserving time-stepping scheme, is adapted to the framework of variational integrators. Specifically, it has been shown that the resulting scheme is not only equivalent to the corresponding scheme using Lagrange multipliers, but that it can be derived itself via a discrete variational principle. The derivation of viable discrete null space matrices is simpler in the variational setting, in fact the discrete null space matrix is simply obtained by evaluating the continuous one at a discrete configuration. This facilitates the solution, in particular the linearization of the discrete equations leading to reduced computational costs for the example of a closed kinematic chain. On the other hand, for the example of flexible multibody dynamics, the advantageous properties of the smaller dimensional and always well conditioned time-stepping scheme emanating from the discrete null space method became obvious for both schemes. However, this example also revealed that the energy-momentum conserving integrator is capable of handling problems with higher stiffness than the variational integrator, although one must say that promising techniques for handling systems with stiff components is still in development in the variational context. Finally it has been 
demonstrated numerically that the discrete trajectories, velocities, momenta and Lagrange multipliers computed by the energy-momentum scheme approach those of the variational integrator and vice versa for decreasing time-steps.

No attempt on comparing the presented constrained formulation of multibody dynamics to other kinematic concepts for tree structured systems as in [53] or [54] is made here. The latter combines a tree representation in generalized coordinates (where parallel linkages and closed loops are handled with holonomic constraints) with a systematic caching technique to reduce computational costs. A comparison is certainly of interest and remains for future work.

Acknowledgements The authors would like to thank P. Betsch and P. Steinmann whose works with the first author on energy-momentum conserving integration of multibody dynamics are cited frequently here. Thanks also to S. Ober-Blöbaum for helpful discussions. Furthermore, the support of the Alexander von Humboldt-Foundation is gratefully acknowledged.

\section{References}

[1] E. Hairer, C. Lubich, and G. Wanner, Geometric Numerical Integration: Structure-Preserving Algorithms for Ordinary Differential Equations (Springer, Berlin, New York, 2002).

[2] B. Leimkuhler and S. Reich, Symplectic integration of constrained Hamiltonian systems, Math. Comput. 63, 589-605 (1994).

[3] B. Leimkuhler and G. W. Patrick, A sympletic integrator for Riemannian manifolds, J. Nonlinear Sci. 6(4), 367-384 (1996).

[4] S. Reich, Momentum conserving symplectic integrators, Physica D 76(4), 375-383 (1994).

[5] R. McLachlan and G. Quispel, Geometric integrators for odes, J. Phys. A 39(19), 5251-5286 (2006).

[6] C. Kane, J. Marsden, M. Ortiz, and M. West, Variational Integrators and the Newmark Algorithm for Conservative and Dissipative Mechanical Systems, Int. J. Numer. Methods Eng. 49, 1295-1325 (2000).

[7] A. Lew, J. Marsden, M. Ortiz, and M. West, An overview of variational integrators, in: Finite Element Methods: 1970s and beyond (CIMNE, Barcelona, Spain, 2003), pp. 85-146.

[8] J.E. Marsden and M. West, Discrete mechanics and variational integrators, Acta Numer. 10, 357-514 (2001).

[9] J. Wendlandt and J. Marsden, Mechanical integrators derived from a discrete variational principle, Physica D 106, 223-246 (1997).

[10] P. Betsch, The discrete null space method for the energy consistent integration of constrained mechanical systems. Part I: Holonomic constraints, Comput. Methods Appl. Mech. Eng. 194(50-52), 5159-5190 (2005).

[11] L. Petzold and P. Loetstedt, Numerical solution of nonlinear differential equations with algebraic constraints II: Practical implications, SIAM J. Sci. Comput. 7(3), 720-733 (1986).

[12] E. Hairer, C. Lubich, and M. Roche, The numerical solution of differential algebraic equations by Runge-Kutta methods (Springer, Berlin, New York, 1989).

[13] S. Leyendecker, P. Betsch, and P. Steinmann, Energy-conserving integration of constrained Hamiltonian systems - a comparison of approaches, Comput. Mech. 33, 174-185 (2004).

[14] P. Betsch and S. Leyendecker, The discrete null space method for the energy consistent integration of constrained mechanical systems. Part II: Multibody dynamics, Int. J. Numer. Methods Eng. 67(4), 499-552 (2006).

[15] S. Leyendecker, Mechanical integrators for constrained dynamical systems in flexible multibody dynamics, PhD thesis, University of Kaiserslautern, 2006.

[16] S. Leyendecker, P. Betsch, and P. Steinmann, The discrete null space method for the energy consistent integration of constrained mechanical systems. Part III: Flexible multibody dynamics, Multibody Syst. Dyn. 19, 45-72 (2008), DOI 10.1007/s11044-0079056-4.

[17] R. Abraham, J. Marsden, and T. Ratiu, Manifolds, Tensor Analysis, and Applications (Springer, New York, 1988).

[18] J. Angeles and S. Lee, The modeling of holonomic mechanical systems using a natural orthogonal complement, Trans. Can. Soc. Mech. Eng. 13(4), 81-89 (1989).

[19] G. Fischer, Lineare Algebra (Vieweg, Braunschweig, 1997).

[20] O. Gonzalez, Time Integration and Discrete Hamiltonian Systems, J. Nonlinear Sci. 6, 449-467 (1996).

[21] P. Betsch and P. Steinmann, Conserving properties of a time FE method - Part III: Mechanical systems with holonomic constraints, Int. J. Numer. Methods Eng. 53, 2271-2304 (2002).

[22] H. Heuser, Funktionalanalysis (Teubner, Stuttgart, 1992).

[23] B. Schmidt, S. Leyendecker, and M. Ortiz, $\Gamma$-convergence of variational integrators for constrained systems (2007), submitted for publication.

[24] S. Müller and M. Ortiz, On the gamma-convergence of discrete dynamics and variational integrators, J. Nonlinear Sci. 14(4), 153-212 (2004).

[25] P. Betsch and P. Steinmann, Frame-indifferent beam finite elements based upon the geometrically exact beam theory, Int. J. Numer. Methods Eng. 54, 1775-1788 (2002).

[26] P. Betsch and P. Steinmann, Constrained dynamics of geometrically exact beams, Comput. Mech. 31, 49-59 (2003).

[27] O. Gonzalez, Mechanical systems subject to holonomic constraints: differential-algebraic formulations and conservative integration, Physica D 132, 165-174 (1999).

[28] P. Betsch and P. Steinmann, Constrained integration of rigid body dynamics, Comput. Methods Appl. Mech. Eng. 191, 467-488 (2001).

[29] S. Antmann, Nonlinear Problems in Elasticity (Springer, New York, 1995).

[30] J. Marsden and T. Ratiu, Introduction to Mechanics and Symmetry. A Basic Exposition of Classical Mechanical Systems, Texts in Applied Mathematics 17 (Springer, New York, 1994). 
[31] J. Angeles, Rational Kinematics (Springer, New York, 1988).

[32] J. Wittenburg, Dynamics of Systems of Rigid Bodies (Teubner, Stuttgart, 1977).

[33] J. Lerbet, Coordinate-free kinematic analysis of overconstrained mechanisms with mobility one, Z. Angew. Math. Mech. 85(10), 740-747 (2005).

[34] C. Führer, Differential-algebraische-Gleichungssysteme in mechanischen Mehrkörpersystemen. Theorie, numerische Ansätze und Anwendungen, $\mathrm{PhD}$ thesis, Technische Universität München, 1988.

[35] S. Kim and M. Vanderploeg, QR decomposition for state space representation of constrained mechanical dynamic systems, J. Mech. Trans. Auto. Des. 108, 183-188 (1986).

[36] E. Kreuzer, Symbolische Berechnung der Bewegungsgleichungen von Mehrkörpersystemen (Fortschrittsbericht der VDI Zeitschriften, Düsseldorf, 1979).

[37] P. Schatz, Rhythmusforschung und Technik, 2., erw. Aufl. (Verlag Freies Geistesleben, Stuttgart, 1998).

[38] G. Warburton, The Dynamical Behaviour of Structures (Pergamon, Oxford, New York, 1976).

[39] I. Romero and F. Armero, An objective finite element approximation of the kinematics of geometrically exact rods and its use in the formulation of an energy-momentum scheme in dynamics, Int. J. Numer. Methods Eng. 54, 1683-1716 (2002).

[40] T. Hughes, The Finite Element Method. Linear Static and Dynamic Finite Element Analysis (Dover, New York, 2000).

[41] G. Jelenić and M. Crisfield, Interpolation of rotational variables in non-linear dynamics of 3d beams, Int. J. Numer. Methods Eng. 43, 1193-1222 (1998).

[42] A. Ibrahimbegović and S. Mamouri, Finite rotations in dynamics of beams and implicit time-stepping schemes, Int. J. Numer. Methods Eng. 41, 781-814 (1998).

[43] M. Crisfield and G. Jelenić, Objectivity of strain measures in the geometrically exact three-dimensional beam theory and its finite-element implementation, Proc. R. Soc. Lond. A 455, 1125-1147 (1999).

[44] J. Simo and L. Vu-Quoc, On the dynamics in space of rods undergoing large motions - A geometrically exact approach, Comput. Methods Appl. Mech. Eng. 66, 125-161 (1988).

[45] G. Jelenić and M. Crisfield, Geometrically exact 3d beam theory: implementation of a strain-invariant finite element for statics and dynamics, Comput. Methods Appl. Mech. Eng. 171, 141-171 (1999).

[46] S. Leyendecker, P. Betsch, and P. Steinmann, Objective energy-momentum conserving integration for the constrained dynamics of geometrically exact beams, Comput. Methods Appl. Mech. Eng. 195, 2313-2333 (2006).

[47] O. Bauchau, G. Damilano, and N. Theron, Numerical integration of non-linear elastic multibody systems, Int. J. Numer. Methods Eng. 38, 2727-2751 (1995).

[48] A. Ibrahimbegović and S. Mamouri, Energy consering/decaying implicit time-stepping scheme for nonlinear dynamics of threedimensional beams undergoing finite rotations, Comput. Methods Appl. Mech. Eng. 191, 4241-4258 (2002).

[49] J. C. Simo and O. Gonzalez, Assessment of Energy-Momentum and Symplectic Schemes for Stiff Dynamical Systems, in: Proceedings of the ASME Winter Annual Meeting (American Society of Mechanical Engineers, New Orleans, Louisiana, 1993)

[50] A. Lew, J. Marsden, M. Ortiz, and M. West, Asynchronous variational integrators, Arch. Rat. Mech. Anal. 167, 85-146 (2003).

[51] A. Lew, J. Marsden, M. Ortiz, and M. West, Variational time integrators, Int. J. Numer. Methods Eng. 60, 153-212 (2004).

[52] P. Du Toit, I. Mezić, and J. Marsden, Coarse Variables for a Perturbed Coupled-Oscillator Model (2008), submitted for publication.

[53] R. Featherstone, Robot Dynamics Algorithms (Kluwer Academic Publishers, Norwell, 1987).

[54] E. Johnson and T. Murphey, Scalable variational integrators for constrained mechanical systems in generalized coordinates (2008), submitted for publication. 